\title{
Spectroradiometry with Space Telescopes
}

\author{
Anuschka Pauluhn • Martin C.E. Huber • Peter L. \\ Smith • Luis Colina
}

accepted for publication in AARev 29 Sept 2015

\section{Contents}

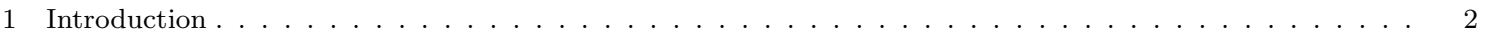

2 Laboratory basis for spectroradiometry and its transfer to space . . . . . . . . . . . . . . . . . 7

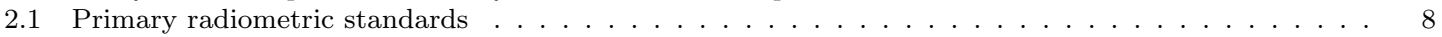

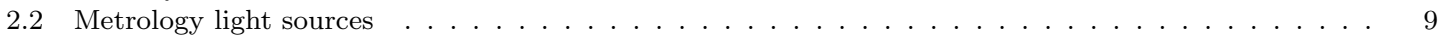

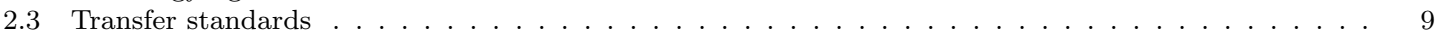

2.4 Radiometric calibration of spectroscopic space telescopes . . . . . . . . . . . . . . . . 10

2.5 Monitoring spectoradiometric response in space . . . . . . . . . . . . . . . . . 11

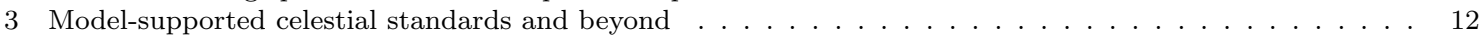

3.1 Vega, Sirius and the process of radiometrically calibrating the Hubble Space Telescope . . . . . . . 15

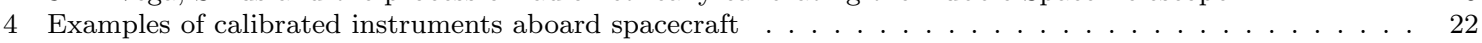

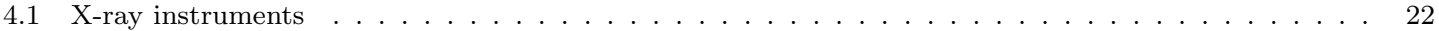

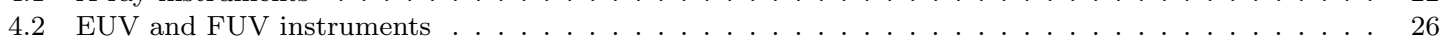

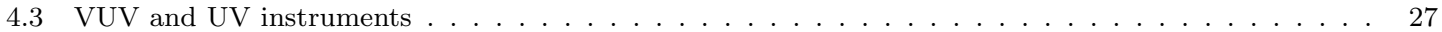

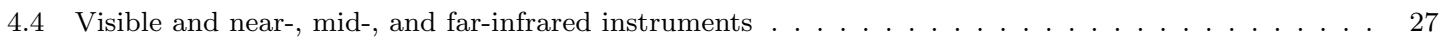

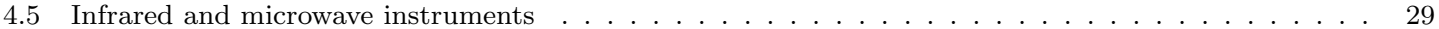

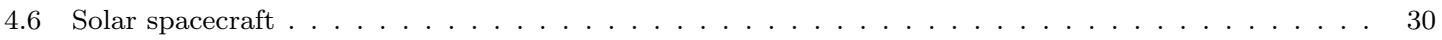

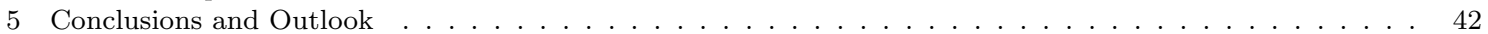

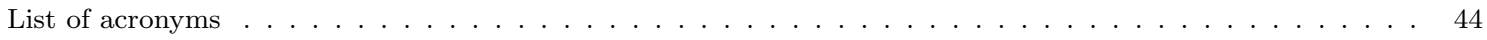

\begin{abstract}
Radiometry, i.e., measuring the power of electromagnetic radiation - hitherto often referred to as "photometry" - is of fundamental importance in astronomy. We provide an overview of how to achieve a valid laboratory calibration of space telescopes and discuss ways to reliably extend this calibration to the spectroscopic telescope's performance in space. A lot of effort has been, and still is going into radiometric "calibration" of telescopes once they are in space; these methods use celestial primary and transfer standards and are based in part on stellar models. The history of the calibration of the Hubble Space Telescope serves as a platform to review these methods. However, we insist that a true calibration of spectroscopic space telescopes must directly be based on and traceable to laboratory standards, and thus be independent of the observations.
\end{abstract}

Anuschka Pauluhn · Martin C.E. Huber

Paul Scherrer Institut, 5232 Villigen PSI, Switzerland E-mail: anuschka.pauluhn@psi.ch

Peter L. Smith

Harvard-Smithsonian Center for Astrophysics, Cambridge MA 02138, USA

Luis Colina

Centro de Astrobiología (CSIC/INTA) 28850 Torrejón de Ardoz, Madrid, Spain 
This has recently become a well-supported aim, following the discovery of the acceleration of the cosmic expansion by use of type-Ia supernovae, and has led to plans for launching calibration rockets for the visible and infrared spectral range. This is timely, too, because an adequate exploitation of data from present space missions, such as Gaia, and from many current astronomical projects like Euclid and WFIRST demand higher radiometric accuracy than is generally available today.

A survey of the calibration of instruments observing from the X-ray to the infrared spectral domains that includes instrument- or mission-specific estimates of radiometric accuracies rounds off this review.

\section{Introduction}

The advent of spectroscopy in astronomy led, about 100 years ago, to the development of astrophysics. Today, our knowledge of the Universe is, to a significant extent, based on spectroradiometric 1 observations, i.e., on the determination of the spectral irradiance or spectral radiance ${ }^{2}$ Such measurements require determining the number as well as the spectral and spatial (or rather directional) distributions of photons that arrive at an observer's telescope from an object. When combined with atomic and molecular data and correct distances, spectroradiometric observations provide the essential evidence for deriving temperatures, densities, gravity, element abundances, ionisation stages, and flow and turbulent velocities in objects that are in the gaseous or plasma state. In this way, we elucidate their physical structures, chemical compositions, and the processes that cause them to emit (see, e.g., Wilhelm and Fröhlich 2013). Such observations also provide similar inferences about dust and solid bodies in interplanetary, interstellar, and intergalactic space.

The history of astronomy shows that observations with an improved accuracy of measurement frequently uncover flaws in our knowledge of celestial objects. Usually, this process leads to a more detailed and comprehensive understanding of the object under study. In some instances - the discovery by Penzias and Wilson (1966) of the cosmic microwave background, for example - radiometric measurements of improved accuracy uncover hitherto hidden phenomena in backgrounds, foregrounds, and surroundings that are also observed.

Findings from astronomical observations thus critically depend upon knowledge of the calibration of the data with respect to primary laboratory radiance standards (cf., Sect. 2). Because rigourous science requires rigourous measurements, astronomers should ensure that their spectroradiometric observations are traceable to such standards through frequent, thorough, spectroradiometric calibration. This implies judging the uncertainty of the effective area ${ }^{3}$ of the telescopespectrometer combination as a function of wavelength or energy of their observation.

\footnotetext{
1 In astronomy, the term photometry is often used when dealing with broadband light-level measurements; those with higher spectral resolution are called spectrophotometry. However, in general terminology of radiation measurements, photometry refers to intensity determinations that are relevant to human vision. Therefore, we have chosen to use the terms radiometry or spectroradiometry, which apply to measurements in the entire electromagnetic spectrum, in this paper. To avoid confusion, we use the term "(astronomical) photometry" when discussing results of the photometry method of astronomy.

2 If an object is not spatially resolved, irradiance, $I$, the detected power per unit area (with unit symbol W $\mathrm{m}^{-2}$; often, loosely, called radiative flux) is measured. Spectral irradiance refers to the irradiance per energy (or wavelength) interval at a given energy (or wavelength). Radiance, $R$, is the power per unit area per unit solid angle, with unit symbol $\mathrm{W} \mathrm{m}^{-2} \mathrm{sr}^{-1}$ (cf., The International System of Units (SI), Brochure, 8th edition (2006, updated in 2014), Bureau International des Poids et Mesures (BIPM) http://www.bipm.org/en/publications/si-brochure). Spectral radiance refers to the radiance per energy (or wavelength) interval at a given energy (or wavelength), with unit symbol $\mathrm{W} \mathrm{m} \mathrm{m}^{-2} \mathrm{sr}^{-1} \mathrm{eV}^{-1}$ (or $\mathrm{W} \mathrm{m} \mathrm{m}^{-2} \mathrm{sr}^{-1} \mathrm{~nm}^{-1}$ ). If the distance, $d$, to a uniformly emitting object of area, $S$, is known, then the irradiance is related to the radiance by $I=R\left(S / d^{2}\right)$.

3 The effective area of a spectroradiometric instrument is the collecting area of an instrument with loss-free optical elements, i.e., with perfect reflections, diffraction or dispersion efficiency as well as detector efficiency.
} 
Table 1 Wavelength bands as used in this article, together with example missions.

\begin{tabular}{llll}
\hline Name & & nominal range & mission \\
\hline X-ray & & $0.1 \mathrm{keV}$ to $10 \mathrm{keV}$ & XMM-Newton, Chandra, Rhessi, Yohkoh, Suzaku \\
$\quad$ soft X-ray & & $0.1 \mathrm{keV}$ to $3 \mathrm{keV}$ & Yohkoh, Hinode \\
extreme ultraviolet & EUV & $10 \mathrm{~nm}$ to $90 \mathrm{~nm}$ & EUVE, SOHO, TRACE, STEREO, Hinode, SDO, IRIS \\
far ultraviolet & FUV & $90 \mathrm{~nm}$ to $120 \mathrm{~nm}$ & FUSE \\
vacuum UV & VUV & $120 \mathrm{~nm}$ to $200 \mathrm{~nm}$ & IUE, HST \\
ultraviolet & $\mathrm{UV}$ & $200 \mathrm{~nm}$ to $450 \mathrm{~nm}$ & HST \\
visible & & $450 \mathrm{~nm}$ to $750 \mathrm{~nm}$ & HST, SOHO, STEREO \\
near infrared & NIR & $750 \mathrm{~nm}$ to $5 \mu \mathrm{m}$ & HST, AKARI \\
mid infrared & & $5 \mu \mathrm{m}$ to $30 \mu \mathrm{m}$ & JWST \\
far infrared & FIR & $30 \mu \mathrm{m}$ to $100 \mu \mathrm{m}$ & ISO,Spitzer, AKARI, Herschel \\
sub-millimetre & sub-mm & $100 \mu \mathrm{m}$ to $1 \mathrm{~mm}$ & COBE, WMAP,Herschel, Planck \\
\hline
\end{tabular}

The need for precise spectroradiometric calibration has been demonstrated once more by the fact that the uncertainties in the spectral irradiance (often called "flux distribution" in the references) of stellar standards are the dominant systematic error in measuring relative irradiances (often called "relative fluxes" in the literature) of redshifted supernovae Ia and, thus, in determining the nature of the dark energy that is driving the observed accelerating cosmic expansion (Sullivan et al 2011; Bohlin et al 2014).

For spectrometric telescopes in space, current spectroradiometric calibrations are based on a wide variety of techniques whose relationships to primary laboratory standards are not always evident. In this paper we elucidate and critically assess the pedigrees of calibration methods for the X-ray through the sub-millimetre wavelength ranges ${ }^{4}$ An example of a historical pedigree of the calibration of the spectroheliometer on the Apollo Telescope Mount (ATM) of the Skylab mission is given in Figure 1 .

The calibration pedigree for the NIRSpec instrument on the James Webb Space Telescope $(J W S T)$ in Figure 2 looks less complex, as it can rely directly on a primary standard. In the vacuum ultraviolet domain the primary standard would now be synchrotron radiation from a storage ring, which at the time of the calibration presented in Figure 1 had not yet been available.

The nomenclatures for wavelength bands are often arbitrary and not consistent from author to author; we therefore list in Table 1 our preferences, and some of the representative space missions flown more recently carrying instruments for the corresponding bands. Although the focus of this review is on spectrometric telescopes, we include a number of solar irradiance measurements, both spectral and total. These are among the best calibrated measurements from spacecraft and, therefore, provide a model for accurate astronomical spectroradiometric observations.

We consider the laboratory bases for radiometric calibration, discuss the transfer of the calibration to satellite instruments through measurements as well as the establishment of "celestial standards" 5 and point to the spectroradiometric calibration of some selected astronomical spacecraft and their instruments.

Because recent and future space missions will provide data on the Cosmos to an exquisite accuracy, the need for thorough calibration before, and continued monitoring after, launch of spectroscopic instrumentation has been receiving increased attention for several missions lately.

\footnotetext{
4 We leave out the gamma-ray and radio regimes which have their own, markedly different calibration methods (see, e.g., Kanbach et al 2013 Schönfelder and Kanbach 2013).

5 These should not be confused with the primary laboratory standards described in Sect. 2.1.
} 


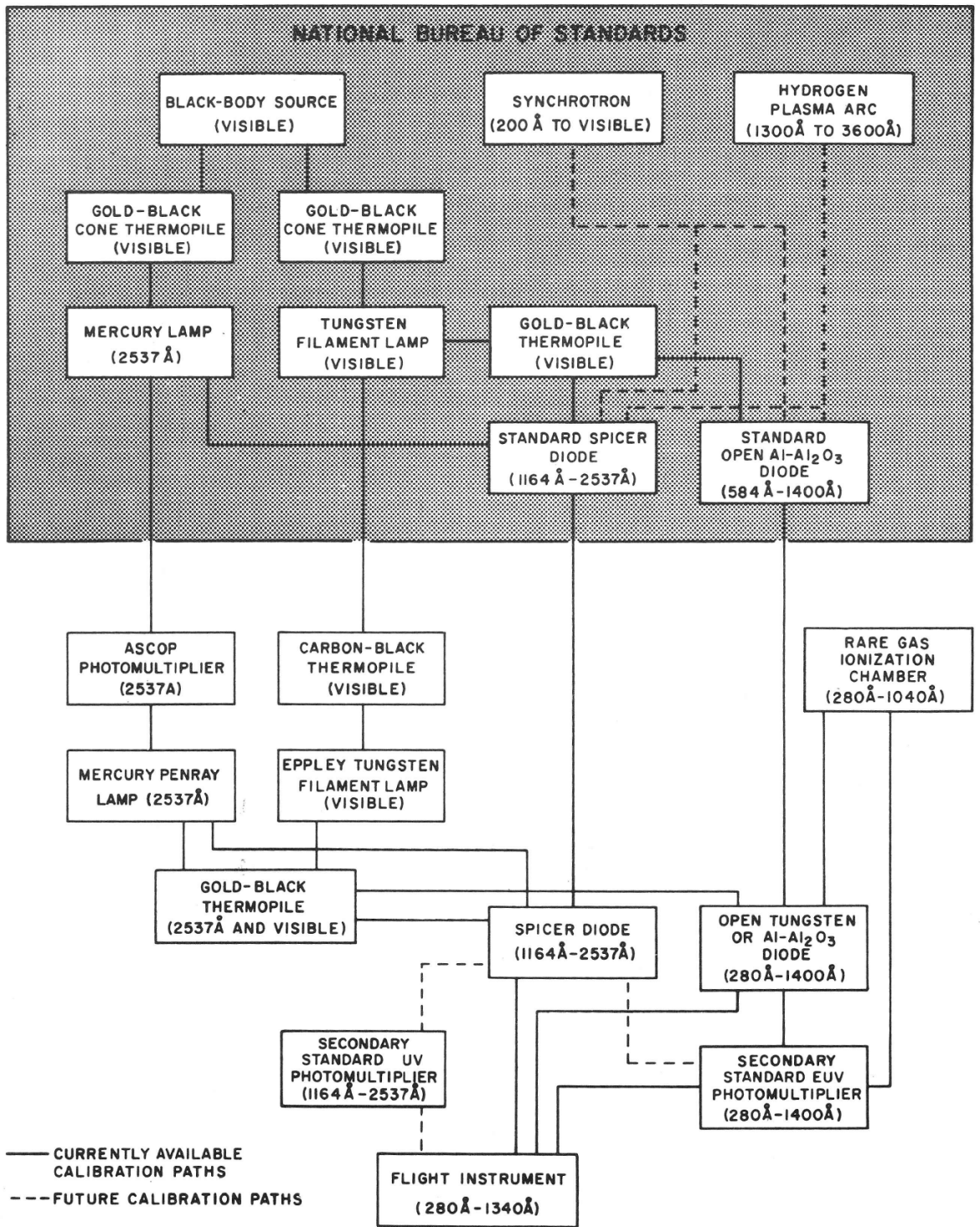

Fig. 1 Pedigree of the calibration of the photoelectric Harvard S-055 spectroheliometer on the Apollo Telescope Mount (ATM) of the Skylab mission (Huber et al 1974).

The Gaia mission ${ }^{6}$ for example, will provide a global stellar census of our Galaxy, which will result in the measurement of distances for more than 20 million stars up to a few kiloparsec away from Earth to one-percent accuracy (Lindegren et al 2008). A one- to three-percent radiometric accuracy would be compatible with measurements on such stars (cf., Pancino et al 2012).

For a duration of six years, starting ca. 2020, the Euclid mission will map at least $35.6 \%$ of the entire sky to derive the geometry and evolution of the dark universe. This will place stringent constraints on dark energy, dark matter, gravity, and cosmic initial conditions (Amendola et al 2013). Unprecedented radiometric precision and accuracy will be required: the relative radiometric

6 Gaia was launched on 19 December 2013 and began its five-year science phase on 29 July 2014. For its progress follow http://sci.esa.int/gaia 


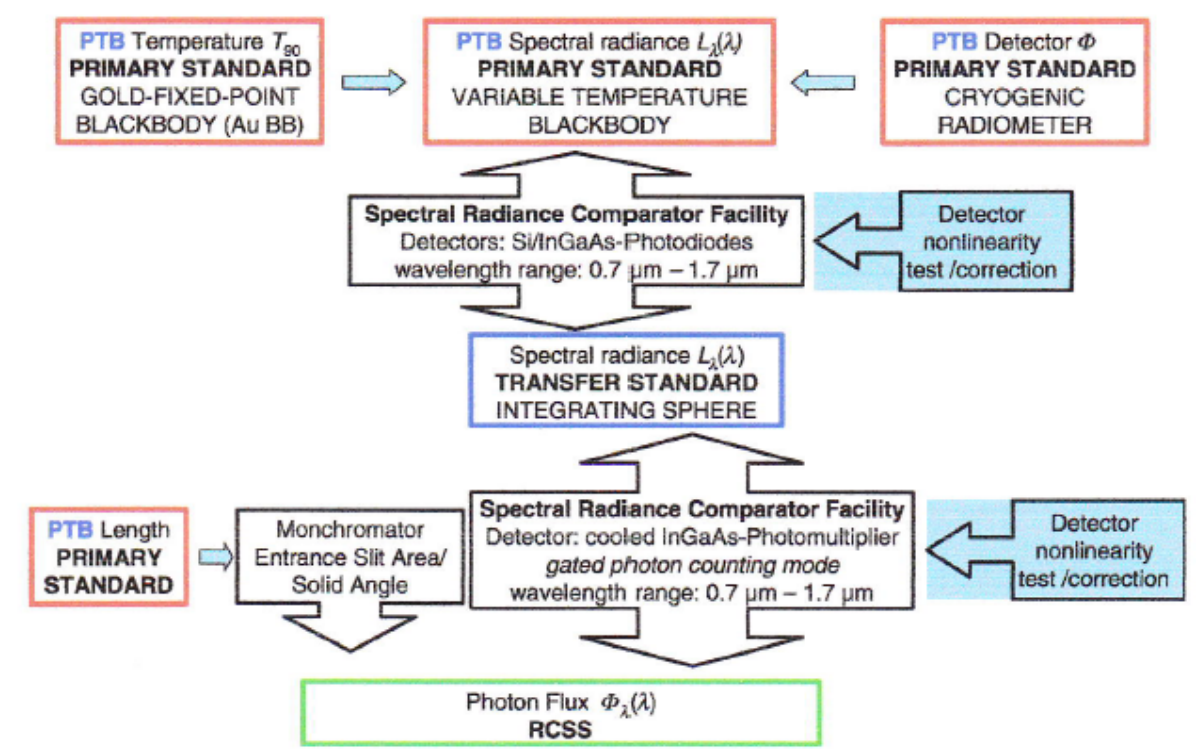

Fig. 2 Calibration pedigree for the radiometric calibration spectral source (RCSS), a very low photon flux radiation source operated under vacuum and cryogenic conditions. It will be used for testing and calibrating the near-infrared multi-object dispersive spectrograph NIRSpec, to be flown on the JWST, in the wavelength range $0.7 \mu \mathrm{m}$ to $5 \mu \mathrm{m}$ (Taubert et al 2009).

uncertainty in imaging with its Near-infrared Spectrograph and Photometer (NISP), for example, shall be less than $0.5 \%$, and it is a mission goal to obtain an absolute radiometric calibration better than $3 \%$ (Laureijs et al 2012).

Moreover, it will be a major challenge to maintain a stable radiometric response on ESA's Solar Orbiter (to be launched in 2018), which will observe the Sun from about 0.15 au, i.e., from half the distance between Mercury and the Sun. In the 1960s already it was noted that the effective area of the normal-incidence vacuum-ultraviolet (VUV) spectroheliometer on OSO-4 changed during laboratory calibration and again before early observations in space (Reeves and Parkinson 1970). Degradation, with spectrally different rates, was registered during further exposure in orbit on $O S O-4$ and on $O S O-6$ (Huber et al 1973). In contrast, no degradation of the responsivity on grazing-incidence spectrometers was found, except in some cases, where the reduction could be ascribed to loss of responsivity of open-structure detectors (cf., Neupert et al 1969 Hinteregger and Hall 1969). Moreover, comparisons between the responsivity of the grazing-incidence spectrometer on $O S O-4$ and a sounding rocket measurement by the University College group (Bowen et al 1971) had shown perfect agreement of the measured irradiance by the satellite and rocket instruments (cf., Figure 3) 7 The responsivity of the follow-on normal-incidence instrument S-055 on the Apollo Telescope Mount on Skylab (Reeves et al 1977a) was therefore monitored by use of a "celestial standard", namely a "quiet area" in the centre of the solar disk, and checked by three calibration-rocket flights (Timothy et al 1975), which simultaneously observed the same "quiet area". The results (Reeves et al 1977b) are shown in Figure 4. This comparison at the same time gives an indication on the errors one may have to contend with, when using a "quiet" area in the centre of the solar disk as "celestial standard" to monitor instrument responsivity. It was generally concluded that the main reason for the loss of sensitivity of normal-incidence VUV instruments

\footnotetext{
7 In spite of grazing-incidence optics being much less sensitive to contamination, and therefore having a better radiometric stability, normal-incidence instrumentation is usually preferred - at least as long as surfaces with sufficient reflectivity are available, because the optical design of normal-incidence optics is considerably less difficult.
} 


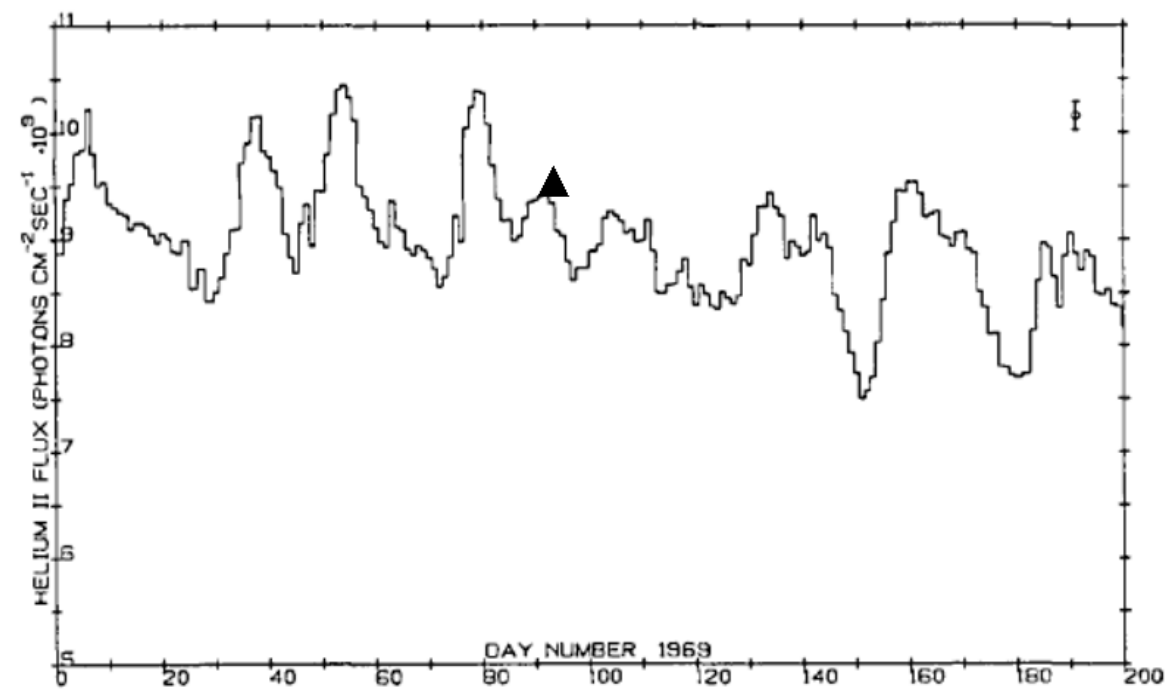

Fig. 3 The long-term solar irradiance variations in the HeII Lyman- $\alpha$ line as measured in 1968 by a grazingincidence spectrometer on the $O S O-4$ satellite. A comparison with the irradiance measured on day 93 by two calibration-rocket spectrometers (black triangle) showed that the responsivity of the satellite spectrometer had not changed (Bowen et al 1971). The variations in the signal stem from the 28-day solar rotation, which makes active regions appear and disappear on the solar disk. The active regions are also waxing and waning, on different time scales.

was polymerisation of minute layers of volatile substances on the optical surfaces. Based on the experiences with other early solar space observatories, a special effort was undertaken to maintain an extensive cleanliness programme for the SOHO instruments and spacecraft (Pauluhn et al 2002). More recently, these kind of studies have been taken up again by BenMoussa et al (2013) who comprehensively analysed the behaviour of numerous instruments designed to observe the Sun in space and drew renewed attention to this problem.

Missions whose primary aim is solar physics can (and will) insist on clean platforms. Monitoring the solar EUV irradiance for use in modelling the thermosphere and the ionosphere, however, is an operational issue that cannot wait until a clean platform becomes available: real-time measurements of the solar VUV irradiance provide data needed for ionospheric modelling that in turn permits correcting propagation delays of navigation signals from space to Earth 8 In an aperçu on the history of VUV solar-irradiance measurements since 1946, Schmidtke (2015) points out that it was only at the end of the 20th century that enough progress had been made to solve the serious problem of degradation. The use of a primary standard in orbit now permits measuring the solar VUV irradiance with sufficient radiometric accuracy. As a consequence, a data set covering a full 11-year solar cycle is now available for the first time.

In their report about measurements over eight years of the VUV solar irradiance by SolACES on the International Space Station $(I S S)$ Schmidtke et al (2014) describe "a very strong up-anddown variability of the spectrometric efficiency by orders of magnitude", which is assumed to stem at least in part from contamination by supply missions. The use of an in-orbit absolute standard nevertheless led to the reliable results now available.

Finally, we should draw attention to the summarising report by Schläppi et al $(2010)$, in which the environments of several spacecraft - and in particular of Rosetta-during their trajectories and

8 By adding airglow and auroral-emission monitoring, the impact of space weather on the terrestrial thermosphere/ionosphere can be studied as well, and used to investigate real-time space weather effects. This in turn helps to derive detailed correction procedures for the evaluation of global navigation satellite system signals. 


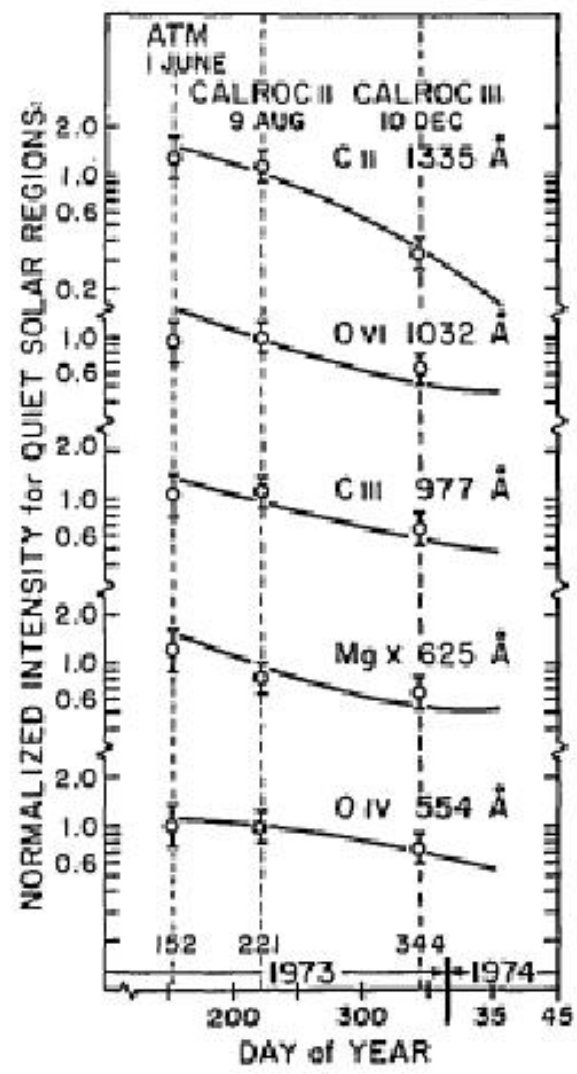

Fig. 4 Comparison of the apparent relative calibration changes of the Harvard S-055 spectroheliometer on the Apollo Telescope Mount (ATM) observed in orbit and the results of the preflight and rocket measurements. (From Reeves et al (1977b)).

operation in space have been studied and interpreted in detail. The authors conclude that "the most important process of outgassing during the first 200 days of a mission is desorption of water from exposed surfaces of the spacecraft." For a spacecraft at $1 \mathrm{au}$, the halflife of the density associated with desorption was observed to be ca. 21 days. After several years in space, decomposition of the material, mostly attributed to solar UV radiation, begins to play a role. The contamination of sensitive surfaces may vary considerably between different spacecraft, and between instruments on the same spacecraft; it can also vary with time, as the spacecraft attitude changes and payload operations take place. In general, shadowed parts contain mostly water, whereas parts that are mostly in the Sun may exhibit a large contribution from decomposition of, for example, lubricants. In spite of strong efforts to avoid contamination by a careful design and selection of materials, there remains a risk of measurements falsified by contamination of optical surfaces. In the long range, as more and more accurate spectroradiometry will be needed to keep pace with progress in astronomy, the use of primary standards in orbit will be required. Source standards will then probably become the preferred kind of standard.

\section{Laboratory basis for spectroradiometry and its transfer to space}

Spectroradiometry that is performed on an astronomical object to derive physical properties must be based on primary laboratory standards, i.e., on absolute radiation sources or detectors 9 The criterion for primary standards is that they give results directly in Système International (SI) units

\footnotetext{
9 A brief summary of Section 2 has been given earlier by Huber et al (2013).
} 
without the need for any calibration relative to the quantity being measured. One must be able to write the complete operating equation for the standard without any unknown or empirically determined constants or functions that depend upon the quantity being measured (Quinn 1997). These standards are realised in the laboratory; $\mathrm{Cook}(1994)$ has discussed the fundamental necessity for this approach.

In this section we discuss the current primary standards for radiometry, ways of applying spectroradiometric calibration to space instruments, and methods for monitoring changes in these calibrations in orbit. In Section 3 we will then discuss celestial standards for astronomical spectroradiometry and the spectra of model stellar atmospheres for white dwarfs. These modelled spectra are used to extend calibration capability beyond the wavelength and irradiance ranges of the astronomical standards.

\subsection{Primary radiometric standards}

Due to the large ranges of intensity and energy (or wavelength) of electromagnetic radiation, practical considerations and current technology have resulted in four primary standards for absolute spectral radiometry: two emission or source standards, viz., black bodies, and electron (or positron) storage rings, and two detector standards, viz., cryogenic radiometers and double-ionisation chambers.

Black bodies (cf., Kaase et al 1984) are emission standards based on thermodynamics: the radiation from a black body is related to its temperature by the Planck law, where the operational temperatures are normally those of melting points of appropriate metals (Hartmann 2009).

Electron (or positron) storage rings (cf., Madden et al 1992) are standards based on electrodynamics. The Schwinger (1949) formula is used to calculate the synchrotron radiation emitted by the accelerated charged particles. Inputs required for the calculation are the energy and current of the stored beam as well as the magnetic induction at the point where the radiation is emitted; small corrections are required to account for the finite vertical extent and divergence of the particle beam.

Cryogenic electrical substitution radiometers (ESR, cf., Möstl 1991) are detector standards that are based on accurate current and voltage measurements. The temperature increase of an irradiated cavity cooled to liquid helium temperatures is compared by a null method with the temperature increase caused by deposition of accurately measured electrical power into an equivalent cavity. Although corrections for reflections, scattering and diffraction must be made, cryogenic radiometers are accepted as the most accurate radiometric standards (Fox and Martin 1990; Foukal et al 1990; Hoyt and Foukal 1991: Quinn and Martin 1991a b).

Rare-gas double-ionisation chambers are detector standards based on the fact that the photoionisation yield of rare gases is unity over the wavelength range $30 \mathrm{~nm}$ to $102 \mathrm{~nm}$ (Samson 1964: Samson and Haddad 1974; Samson and Ederer 2000). Ionisation chambers are the only primary standards that have been used in space to date (Carlson et al 1984; Schmidtke et al 2014), though non-cryogenic ESRs, which are less sensitive and accurate than the cryogenic variety, have been used to measure and monitor the solar constant (see Sect. 4).

The state of agreement between the radiometric scales realised by the first three primary standards mentioned above was discussed by Stock et al (1993). Their general uncertainty analysis indicated that black bodies and cryogenic radiometers produced and detected spectral radiant power with relative uncertainties of $0.08 \%$ and $0.007 \%$, respectively ${ }^{10}$ Thornagel et al (1996) measured emission from an electron storage ring with a cryogenic radiometer and found agreement between the two primary standards within $0.3 \%$ at a photon energy of $15 \mathrm{keV}$ and $0.08 \%$

\footnotetext{
10 Unless otherwise indicated, all uncertainties in this paper are given in terms of one standard deviation, i.e., $68 \%$ confidence limit (coverage factor $k=1$ ).
} 
for visible radiation $\sqrt{11}$ Hollandt et al (2005) have summarised further such comparisons; these included a comparison between synchrotron radiation from the electron storage ring BESSY I with radioactive standards (Arnold and Ulm 1994).

\subsection{Metrology light sources}

Today, user facilities, such as the Metrology Light Source (MLS) at the PTB in Berlin ${ }^{12}$ (Klein et al 2009) or the Synchrotron Ultraviolet Radiation Facility SURF-III ${ }^{13}$ at NIST ${ }^{14}$ (Arp et al 2002) which can accommodate space hardware for radiometric calibrations with synchrotron radiation, are in operation. A dedicated beamline at the MLS in Berlin and a corresponding calibration chamber in a cleanroom - both part of installations that can be used by customers - are shown in Figures 5 and 6 .

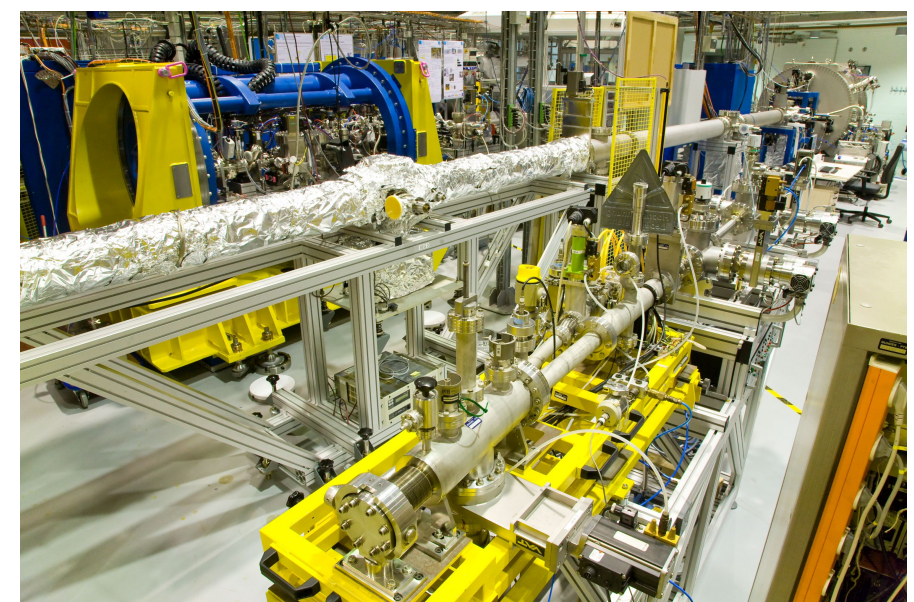

Fig. 5 View from the storage ring downstream at the metrology beamline of the MLS at PTB. The vacuum tank for calibration (also shown in the next figure) is seen at the right upper corner of the image. A transportable VUV transfer source based on a window-less hollow-cathode discharge that has been developed for the calibration of $\mathrm{SOHO}$ instruments in the home laboratories of the Principle Investigators, can also be brought into the beam; it is seen on the lower right side. (From Gottwald et al (2014), with permission by PTB).

\subsection{Transfer standards}

It is often practical to use simple, stable sources and detectors, known as transfer standards, that are themselves calibrated in the laboratory by comparison with the primary standards. Examples

\footnotetext{
11 The Bureau International des Poids et Mesures (BIPM, http://www.bipm.fr) has the mandate to provide the basis for a single, coherent system of measurements - traceable to the International System of Units (SI) - throughout the world. The Bureau was set up by the Convention of the Metre and operates under the exclusive supervision of the Comité International des Poids et Mesures (CIPM). The Committee's principal task is to ensure worldwide uniformity in units of measurement, particularly between national measurement standards, but the CIPM also takes on the more fundamental task to arrange for and monitor comparisons that determine the accuracies with which the individual primary standards are realised.

12 http://www.ptb.de/mls/index.html

13 http://www.nist.gov/pml/div685/grp07/surffacility.cfm

14 the US National Institute of Science and Technology (earlier National Bureau of Standards, NBS)
} 


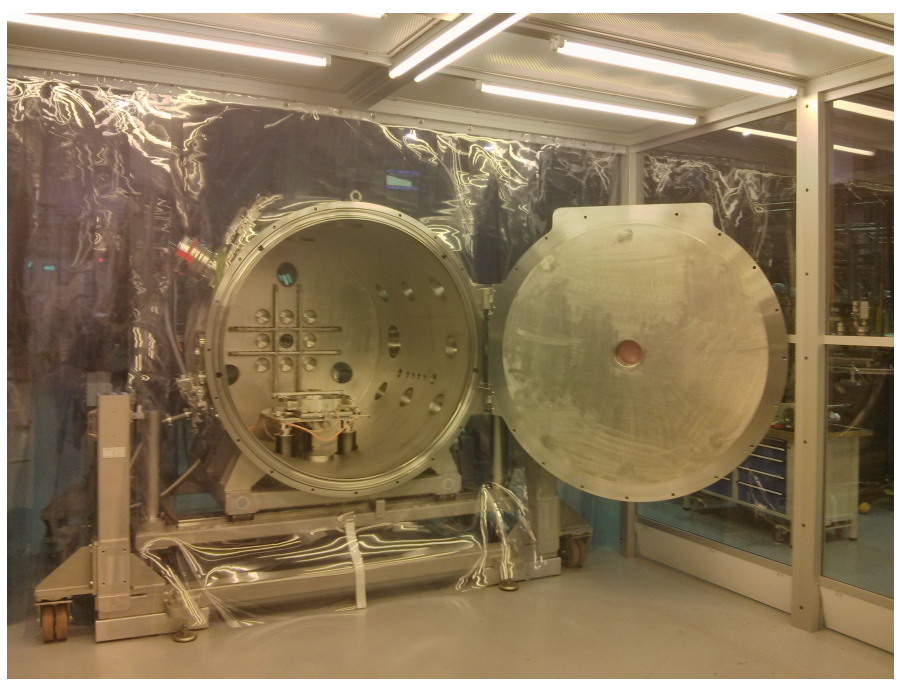

Fig. 6 The vacuum tank for calibration of space instruments at the metrology beamline at the MLS. The tank is embedded in a cleanroom environment to enable the handling of flight hardware directly at the beamline. (From Gottwald et al (2014), with permission by PTB).

include argon arcs (Bridges and Ott 1977), bare metal photoemissive diodes (Saloman 1978), $\mathrm{D}_{2}$ lamps (Klose et al 1988), tungsten-filament incandescent lamps (Waters et al 1988), hollow-cathode (Danzmann et al 1988; Hollandt et al 1994) and Penning (Heise et al 1994) discharges, and silicon trap detectors (Goebel et al 1996) and photodiodes (Kuschnerus et al 1998; Canfield et al 1998).

\subsection{Radiometric calibration of spectroscopic space telescopes}

The procedures used for radiometric calibration of spectrometric space telescopes may be divided into four categories:

(i) Pre-flight calibration in the laboratory at the component and/or subsystem levels or in end-toend (i.e., from entrance aperture to detector output) tests using primary or transfer standards. This approach was followed for the spectroheliometers on the ESA/NASA Solar and Heliospheric Observatory SOHO (Fleck et al 1995) and for the X-ray missions Chandra (Weisskopf et al 2000) and XMM-Newton (Jansen et al 2001).

(ii) Calibration in orbit by celestial standards once these are established through observations or by theoretical models (Bohlin et al 2014). For example, the calibration of the Hubble Space Telescope HST (Bohlin 2007) or of the solar Interface Region Imaging Spectrograph IRIS have been largely done this way (De Pontieu et al 2014). Several instruments on SOHO, in particular SUMER and UVCS, have used stars and planets for monitoring the radiometric calibration or to extend the calibration range to longer wavelengths where no ground calibration was available (Schühle et al 2000 Lemaire 2002: Gardner et al| 2002).

(iii) Calibration of an orbiting spectrometric telescope by comparing its observations with simultaneous ones made by a similar "underflight" instrument on a sub-orbital rocket or the Space Shuttle. The latter instrument is then recalibrated in the laboratory after use in space to minimise uncertainties stemming from changes in effective area. As mentioned above, the radiometric calibration of the ATM spectroheliometer on Skylab has been checked three times with this method (Timothy et al 1975; Reeves et al 1977b), see also Figure 4 . The radiometric accuracy of observations with the Hopkins Ultraviolet Telescope (HUT, Davidsen et al 1992) 
was established in this way and they were then used to validate modelled spectra used as celestial standards (Kruk et al 1997). The Solar Extreme Ultraviolet Experiment (SEE) on NASA's TIMED satellite also used three sounding-rocket flights to follow changes in its radiometric calibration. Rocket underflights have also been used extensively for monitoring the calibration and performance of the $S O H O$ VUV instruments. In particular, they have been extremely useful to track changes after $S O H O$ 's temporary loss of attitude in 1998, see, e.g., Wilhelm (2006) and references therein.

(iv) Calibration by use of a primary or transfer standard that is carried into and operated in orbit as part of the scientific payload ${ }^{15}$ Standards carried into orbit can seldom be used to assure an end-to-end calibration, but are employed for monitoring the stability of a crucial part of an instrument, such as a detector. An exception is the Midcourse Space Experiment (MSX), which performed in-orbit infrared radiometry by releasing calibrated $2 \mathrm{~cm}$ diameter spheres, whose size and emissivity had been precisely measured in the laboratory, and whose temperature could be determined to $\pm 1 \mathrm{~K}$ (Price et al 2004). Instruments devoted to studying total solar irradiance, such as the Variability of Solar Irradiance and Gravity Oscillations (VIRGO) experiment on $S O H O$ and the Spectral Irradiance Monitor (SIM) on the SORCE satellite do use electrical substitution radiometers to directly calibrate their measurements in space ${ }^{16}$ Moreover, rare-gas ionisation chambers, which are primary radiometric detector standards, are operating in the SolACES experiment (Schmidtke et al 2014).

Nowadays, a combination of the described techniques is often used. However, in view of: $(i)$, the multitude of observing modes and the concomitant large parameter space that should be covered during a calibration, $(i i)$, concerns about the differences between conditions during ground testing and those of in-orbit operations, and ( iii), the inevitable changes in performance over time, it has become common practice to employ instrument models that use well-characterised properties of optical components, coatings, and contaminants to interpolate between measured benchmark values of instrument performance and to predict time behaviour (cf., Rosa 1997; Ballester and Rosa 1997, and discussion of Chandra and XMM-Newton in Sect. 4).

\subsection{Monitoring spectoradiometric response in space}

Changes in the effective areas of spectrometric telescopes on spacecraft can be caused by exposure to contaminating pre-launch environments and material outgassed from the instrument or spacecraft itself. Unless extreme care is exercised, degradation can take particularly dramatic forms for solar telescopes, which are directly exposed to the harsh electromagnetic and energetic particle emission of the Sun.

It is difficult to detect whether changes in spectroradiometric efficiency have occurred between laboratory measurements and first light in orbit unless an on-board calibration source that tests the overall system from end to end or reliable celestial standards are available. In order to monitor changes that occur in space, users of spaceborne spectrometers often define an appropriate ensemble of non-variable objects that are observed at regular intervals. However, this procedure is not without complications for solar and X-ray telescopes where the objects observed are variable; on-board calibration systems or underflights remain the best options in these cases. Regular measurements of common targets with several instruments in common wavelength ranges in specifically designed

\footnotetext{
15 An additional concept, viz., having emission transfer standards on the International Space Station (ISS), or on a small calibration satellite orbiting the Earth, with the purpose of calibrating co-orbiting satellites in the extreme-ultraviolet range has been suggested (Smith et al 1991).

16 In contrast to the cryogenic electrical substitution radiometers, where corrections covering non-ideal performance nearly vanish owing to the low operating temperature, ESRs used at higher temperatures require a careful evaluation of outside influences, such as heating of baffles. Besides, for ESRs to be used in space, such tests are complex.
} 
campaigns have proven extremely useful as well. A recent account of in-flight monitoring of the performance of solar instrumentation has been given by BenMoussa et al (2013).

\section{Model-supported celestial standards and beyond}

Spectroradiometry - from the VUV through the NIR - of astronomical night-sky objects relies most commonly on the radiometric calibration of the spectra of a small set of stars, the so-called celestial primary standards - with Vega $(\alpha$ Lyr) as the original primary standard. The state-ofthe-art calibration of these celestial standards is based on direct, ground-based comparisons of the irradiance of Vega with that of laboratory standards, viz. black bodies or calibrated lamps, at the wavelength of $555.6 \mathrm{~nm}$ (Oke and Schild 1970; Hayes and Latham 1975, Tüg et al 1977; Hayes 1985; Mégessier 1995) ${ }^{17}$ The calibration was then extended to other wavelength domains by stellar atmosphere models. This was rather successful when going to shorter wavelengths by use of model spectra of hot white dwarfs (WD) with pure hydrogen atmospheres. Calibration in the infrared to $35 \mu \mathrm{m}$ was established in a series of 14 papers in the Astronomical Journal that was initiated by Martin Cohen in 1992 (Cohen et al 1992a). This effort involved measurements from the ground, by the Kuiper Airborne Observatory, by the Low Resolution Spectrometer on the IRAS satellite, and by stellar atmosphere programmes (see, e.g., Cohen et al 1992b, 2003).

Three additional papers in this series, by Price et al (2004), Engelke et al $(2006)$, and Engelke et al (2010) reported a physics-related calibration performed in orbit (the details of which will be discussed below) and associated improvements to the catalogue of standard stars. Engelke et al (2010) extended the spectral range covered by their standard stars to shorter wavelengths, i.e., to $350 \mathrm{~nm}$. Rieke et al (2008) published another extensive report on calibration in the infrared; they had determined an absolute radiometric calibration for the $24 \mu \mathrm{m}$ band of the Multiband Imaging Photometer on Spitzer, and recommended adjustments to calibrations of the Infrared Array Camera on Spitzer (Fazio et al 2004), to the (ground-based) Two Micron All Sky Survey (2MASS), and to the IRAS (astro)photometry. Rieke et al also showed that integrating measurements of the Sun with those of solar-type stars led to an accurate estimate of the solar irradiance spectrum from $1 \mu \mathrm{m}$ to $30 \mu \mathrm{m}$ that appears to agree with theoretical solar models.

Blackwell and his group at Oxford, who had been performing accurate astronomy-related measurements in the 1970s - originally with measurements of oscillator-strengths for abundance determinations and concomitant testing of stellar atmosphere models and nuclear astrophysic: ${ }^{18}$ - later determined Vega's irradiance at the Observatorio del Teide out to the infrared at $\lambda=4.92 \mu \mathrm{m}$ by comparing it with that of a furnace (cf., for example, Mountain et al 1985; Booth et al 1989).

Observations of Vega by the IRAS satellite (Neugebauer et al 1984) that went further into the infrared have shown that Vega's spectrum exhibits an infrared excess beyond $12 \mu \mathrm{m}$, because solid particles form a warm surrounding of this star (Aumann et al 1984). Moreover, a decade ago, an interferometric study revealed that $\alpha$ Lyrae is a fast rotator seen almost pole-on (Peterson et al 2006). Such deviations from model assumptions of the environment of Vega and of the behaviour of the star itself brought home the general danger of trying to rely on a stellar model and under-

\footnotetext{
17 Spectroradiometric calibrations - at the time called determining the "spectral energy distribution" (SED) of stars - had been performed starting in the 1910s with visual and photographic comparisons with assumed stellar models. Direct comparisons with calibrated standard lamps started at the end of the 1930s at Ann Arbor and continued in the 1940s with extensions to the infrared, and by going to higher altitude, for example at Jungfraujoch, into the ultraviolet as well. The results were usually expressed as colour temperatures and in magnitudes (see, for example, Code 1960).

18 The Oxford group had been working on precision laboratory astrophysics early on. In the 1970s they experimentally determined transition probabilities with one-percent accuracies, when such measurements normally had uncertainties of $\pm 10 \%$ or more. Not surprisingly then, Blackwell chose the topic of "Uncertainty in Astronomy" for his Presidential Address to the Royal Astronomical Society (Blackwell 1975).
} 
lined the care that should be exercised in choosing a standard star if one wanted to extrapolate radiometric properties to hitherto uncalibrated wavelength regions.

Engelke et al (2010) have described the calibration history of (astro) photometry that has been associated with Vega 19 They analysed the voluminous work that had gone into testing Vega's use as a celestial primary standard in the wavelength range between $350 \mathrm{~nm}$ and $35.0 \mu \mathrm{m}$ and concluded that Vega should be replaced as primary celestial standard for (astro)photometry by 109 Vir in the visible and by Sirius ( $\alpha \mathrm{CMa})$ in the infrared. Engelke et al also used results of a direct calibration of infrared irradiances in space by the Midcourse Space Experiment. As part of the MSX, Price et al (2004) have compared the irradiance of a number of infrared standard stars with that of five emissive reference spheres that were ejected at various times during the mission. They describe the calibration properties of the released spheres as follows: "The physical properties of the $2 \mathrm{~cm}$ diameter spheres, such as size and emissivity, had been precisely measured in the laboratory. The energy balance equation between the total flux absorbed and that emitted by the sphere is solved to obtain the time-dependent temperature of the sphere under the assumption that the sphere radiates as a black body with the measured wavelength-dependent emissivity." This resulted in absolutely calibrated values with a claimed standard uncertainty in the one-per-cent range for five wavelengths between $4.3 \mu \mathrm{m}$ and $21.3 \mu \mathrm{m}$.

Based on a comparison of data from MSX measurements with data from the Short Wavelength Spectrometer of ESA's Infrared Space Telescope (ISO, Kessler et al 1996), Engelke et al (2006) created new spectra for stars that had earlier been selected as standards. These new spectra led to model temperatures and stellar angular diameters that, on the one hand, compared favourably with independent measurements (cf., e.g., Mozurkewich et al 2003), but, on the other hand, led to irradiances that were lower by $4 \%$ to $7 \%$ at wavelengths between $1 \mu \mathrm{m}$ and $4 \mu \mathrm{m}$ than those found earlier by Cohen.

Figure 7 shows a comparison between the physics-related stellar irradiances mentioned above with a model-derived stellar energy distribution (SED). The uncertainties of the ground- and spacebased measurements are shown. Those of the tracings of the SED, although not shown, are assumed to be smaller than those of the direct measurements. Clearly, using a stellar atmosphere model to interpolate between physics-related measurements is a pragmatic approach, but inherently retains the danger of circular conclusions if other models are later to be compared with this model.

In order to put the calibration of stars on a solid, physics-based procedure, NIST has developed a Telescope Calibration Facility (TCF) with the aim of achieving SI-traceable spectroradiometric measurements of stellar irradiances with an accuracy of $0.5 \%$ (so-called "SI stars") for groundbased and space-borne observations (Smith et al 2009). This approach has been made possible not only by improvements in radiometry itself but also by advances in measuring and modelling the interfering effects of the terrestrial atmosphere.

The TCF measurements make use of a redundant system of calibrated detectors and sources. Initially, a reference telescope-spectrometer system is calibrated in the Telescope Calibration Facility. This reference telescope is taken to a large astronomical telescope, and both these telescopes then observe a distant calibrated source. If, in a next step, both telescopes measure a given star's irradiance, they should also obtain the same measured value.

This value is, however, still only the "ground-level irradiance" rather than the value that prevails above the atmosphere. A further step is then taken to assess and subsequently eliminate the influence of the atmosphere above the telescopes. To determine the transmittance of the atmosphere one uses inputs from both LIDAR and atmospheric modelling. Assuming stable stars, repeated measurements under different atmospheric conditions will then help to assess the uncertainty caused by

\footnotetext{
19 In reviewing the literature relating to a potential variability of Vega, Bohlin $(2014)$ came to the conclusion that there was not enough support for this claim. Nevertheless, Butkovskaya et al (2011) and Butkovskaya (2014) did not exclude a long-term variability of Vega. The conclusion was that a (very minor) 21-year variability is "most probable". Böhm et al (2015) have recently observed starspots on Vega.
} 


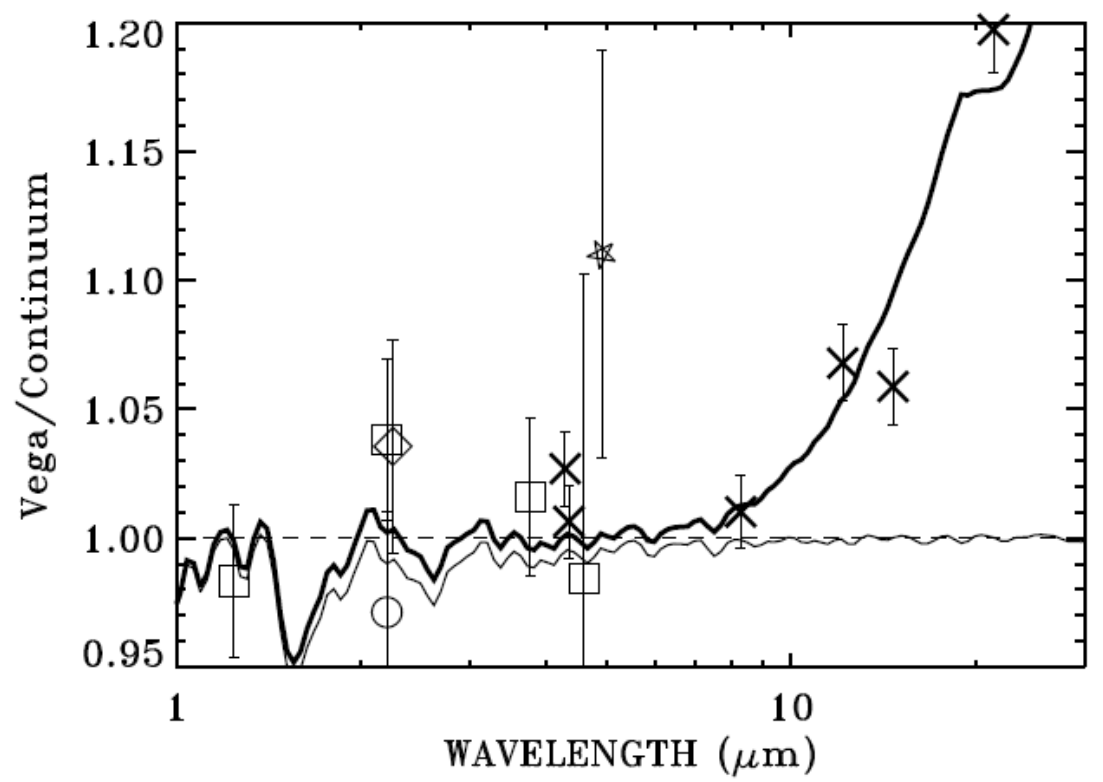

Fig. 7 Comparison of measured infrared absolute irradiances with the modelled stellar energy distributions derived by Bohlin (2014). The light line represents a model with a photospheric temperature of $9400 \mathrm{~K}$ at a resolution of $\mathrm{R}=20$; the heavy line includes the emission from the dust ring. The physics-based measurements obtained on the ground by Selby et al (1983) are represented by a circle, those by Booth et al (1989), by Mountain et al (1985) and by Blackwell et al (1983) by a diamond, a star and squares, respectively. The crosses display measures obtained 20 years later in space by Price et al (2004) from the MSX. (After Figure 9 in Bohlin et al (2014), with permission.)

correcting for atmospheric transmittance. In this way, it is hoped that the "above-the-atmosphere" irradiance of standard stars prevailing in space can be established with sub-percent accuracy for $1 \mathrm{~nm}$ resolution elements over the wavelength interval $350 \mathrm{~nm}$ to $1.05 \mu \mathrm{m}$ (McGraw et al 2012).

In preparation for the US National Academy's "Astro2000" Decadal Survey, Kent et al (2009) wrote a White Paper entitled "Photometric Calibrations for 21st Century Science", where they criticised the traditional spectroradiometry in astronomy: "Our traditional standard star systems, while sufficient until now, need to be improved and extended in order to serve future astrophysics experiments." They therefore advocated "a program to improve upon and expand the current networks of spectrophotometrically ${ }^{20}$ calibrated stars to provide precise calibration with an accuracy of equal to and better than $1 \%$ in the ultraviolet, visible, and near-infrared portions of the spectrum with excellent sky coverage and large dynamic range."

A sub-orbital programme, "ACCESS: Absolute Color Calibration Experiment for Standard Stars" (Kaiser et al 2008a), has subsequently been approved. This programme aims to transfer irradiance represented by absolute detector standards to additional standard stars with better than $1 \%$ precision over the spectral range $350 \mathrm{~nm}$ to $1.7 \mu \mathrm{m}$ with a spectral resolving power of ca. 500. It is planned to repeatedly fly the ACCESS to directly measure the irradiance of six target stars (including Sirius und Vega), whereby each of the target stars is to be observed by two separate rocket flights to ensure repeatability (Kaiser et al 2014). Comparing the results of TCF (aim \pm $0.5 \%$ ) with those obtained by ACCESS (aim $\pm 1 \%$ ) will hopefully lead to a reliable system of standard stars.

20 "spectroradiometrically" in our terminology 
3.1 Vega, Sirius and the process of radiometrically calibrating the Hubble Space Telescope

Bohlin et al (2014) have presented a well-documented description of the elaborate procedures that have been used to calibrate HST. These have led to today's most advanced database for spectroradiometry from the ultraviolet to the mid-infrared wavelength range (110 $\mathrm{nm}$ to $35 \mathrm{\mu m}$ ), i.e., to the CALSPEC database, which will, in future, support the operation of the James Webb Space Telescope (Bohlin 2014).

The calibration started with the above-mentioned ground-based comparisons of Vega's irradiance at the wavelength of $555.6 \mathrm{~nm}$ with standard light sources and was extended to shorter and longer wavelengths by stellar models. Figure 8 illustrates the current r.m.s. uncertainty in Vega's irradiance resulting from the use of models over the wavelength range $100 \mathrm{~nm}$ to $25 \mu \mathrm{m}$

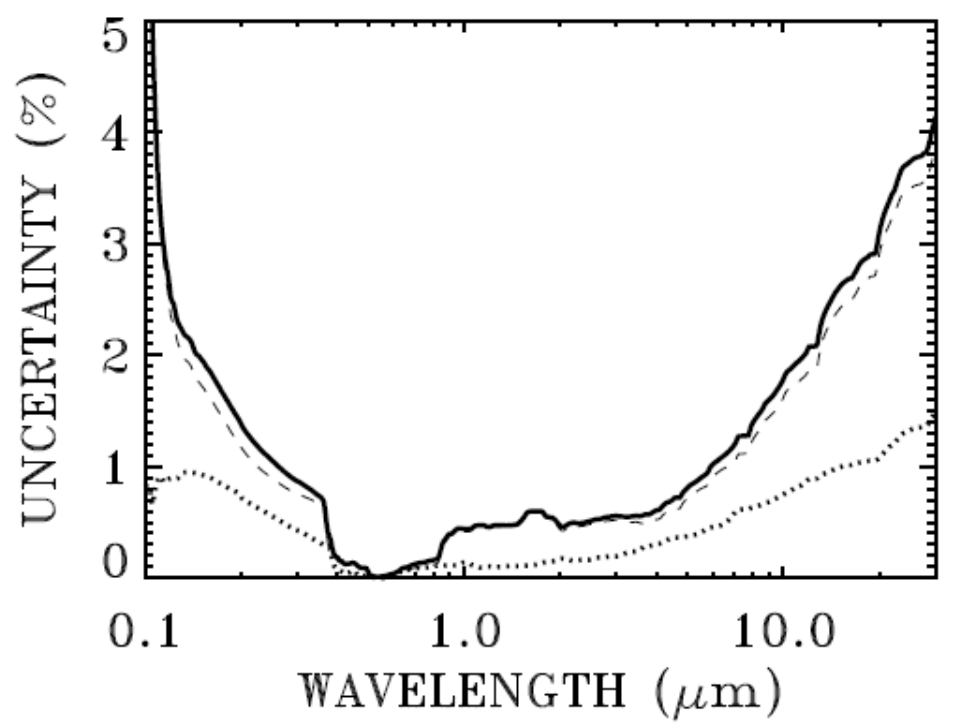

Fig. 8 The uncertainty of the irradiance (beyond that owing to the ground-based comparison with a laboratorybased standard at $555.6 \mathrm{~nm}$ ) for various model assumptions. Dotted line: r.m.s. uncertainty in the white-dwarf irradiance scale from formal errors in the effective temperature used in the original model. Dashed line: The r.m.s. uncertainty as determined from differences between three pairs of each two independently calculated sets of pure hydrogen NLTE models, where the three pairs of models have the same $T_{\text {eff }}$ and $\log g$. Differences arise from errors in one or both sets of calculations that should give the same results. Heavy solid line: Combination in quadrature of these two uncertainties. Owing to the definition of the scale, the relative uncertainty at $555.6 \mathrm{~nm}$ is zero by definition. (After Figure 14 in Bohlin et al (2014), with permission).

As a complete ground-based end-to-end calibration of the $H S T$ telescope and its instruments was not available, Bohlin and his collaborators were facing a gigantic task in spectroradiometrically calibrating this arguably most important space telescope. They chose an in-orbit calibration supported by use of atmosphere models of selected standard stars, supported by physics-related measurements that were available.

This meant compounding the difficulties that one has to contend with after launch of a perfectly calibrated spectrometric telescope, namely

\footnotetext{
21 As pointed out before, Vega has now been replaced by the primary standard stars 109 Vir in the visible and by Sirius ( $\alpha \mathrm{CMa})$ in the infrared, cf., Engelke et al (2010).
} 
- a changing responsivity during space-operations, resulting from

- a changing reflection of optical surfaces,

- changes in detection efficiencies and in non-linearities, as well as

- a degrading of charge-transfer efficiency (CTE) in CCDs, which may also be complicated by a change of performance across the CCD (i.e., a "changing flat-field"),

by the problems inherent in spectroradiometrically calibrating a spectroscopic telescope that had not been fully calibrated before launch by relying on standard stars, namely

- the complications arising, when the spectral resolution of a standard star's spectral energy distribution differs from that of an unknown star,

- partially unknown non-linearities in detectors, and

- scattered light in the spectrometers.

A peculiarity of $H S T$ was that its instruments could be replaced, exchanged or even repaired in orbit. Indeed, astronauts visited the spacecraft on the occasion of five Servicing Missions (SM1 (1993), SM2 (1997), SM3A (1999), SM3B (2002), and SM4 (2009)). Obviously, this brought enormous benefits to the mission, not the least being the replacement of one of the instruments by optics (Bottema 1993) that corrected the initial spherical aberration of the primary mirror. Other benefits were the repair of the Advanced Camera for Surveys (ACS) and of the Space Telescope Imaging Spectrograph (STIS). On the other hand this also required additional efforts for the inorbit calibration of the newly-installed instruments, and prevented continuous monitoring of the radiometric performance of defective instruments. Figure 9 illustrates the change with time in responsivity that the $H S T$ instrument STIS has experienced in orbit: an initial improvement of efficiency and later decay of efficiencies, as well as the occurrence of a data gap. ${ }^{22}$

The description by Bohlin et al (2014) of the HST calibration begins with an in-depth discussion of the comparison of stars to "Laboratory Flux Standards", i.e. "laboratory-pedigreed" stellar irradiances. The initial wavelength range being discussed is the ultraviolet below the atmospheric cut-off at $330 \mathrm{~nm}$; calibration attempts for this wavelength range started in the late 1960s, mostly by rocket flights.

Space astronomers at that time - many of them being experimental physicists - were keen on working with instruments that had a calibration traceable to laboratory standards. Bless et al (1976) judged the initial calibration of the Wisconsin equipment package (Code et al 1970) on NASA's Orbiting Astronomical Observatory (OAO-2) to be rather insecure "because of the long time (18 months) required by spacecraft testing between instrument calibration and launch, and because of the feeble far-ultraviolet light sources available to them in the mid-sixties." They therefore measured the irradiance of $\alpha$ Vir, $\eta \mathrm{UMa}$, and $\alpha$ Leo in several pass bands within the wavelength range $137 \mathrm{~nm}$ to $292 \mathrm{~nm}$ during the flight of an Aerobee sounding rocket.

In a pioneering effort, Bless et al (1976) had calibrated the telescope for (astro)photometry on this sounding rocket by use of synchrotron radiation as standard. The electron storage ring at the

\footnotetext{
22 cf., http://hubblesite.org/the_telescope/team_hubble/servicing_missions.php as well as the caption of Figure 9 Note, however, that HST was not the first scientific satellite to be repaired in orbit. At launch in 1973 the Skylab space station had lost a so-called micrometeorid shield during launch. This shield would also have been a thermal shield of the astronauts' living space. Upon their arrival at Skylab the first crew was able to mount and deploy a heat shade, which saved the mission. In 1984 the Solar Maximum Mission also underwent repair in orbit, when it was visited by the space shuttle Challenger. Another example of a data gap stems from the $S O H O$ mission, which experienced an intermediate time-out as well. Contact with the spacecraft was lost in August 1998 after a sequence of incorrect commands during what should have been a routine manoeuvre. Four weeks later a powerful radar signal from Earth produced a faint echo from the spacecraft indicating that $S O H O$ had not drifted away from its position in its L1-halo orbit after loss of contact. It was slowly rotating and angled in such a way that sunlight was going to fall on its solar cells during the following months. Normal operations could then be resumed after an extended turn-on and test period. The responsivity of many instruments had changed - out-baking in the absence of thermal control had, in fact, improved the responsivity of some instruments (cf., Pauluhn et al 2002). In this context we recall the earlier mentioned reference about the outgassing of a spacecraft after launch (Schläppi et al 2010).
} 


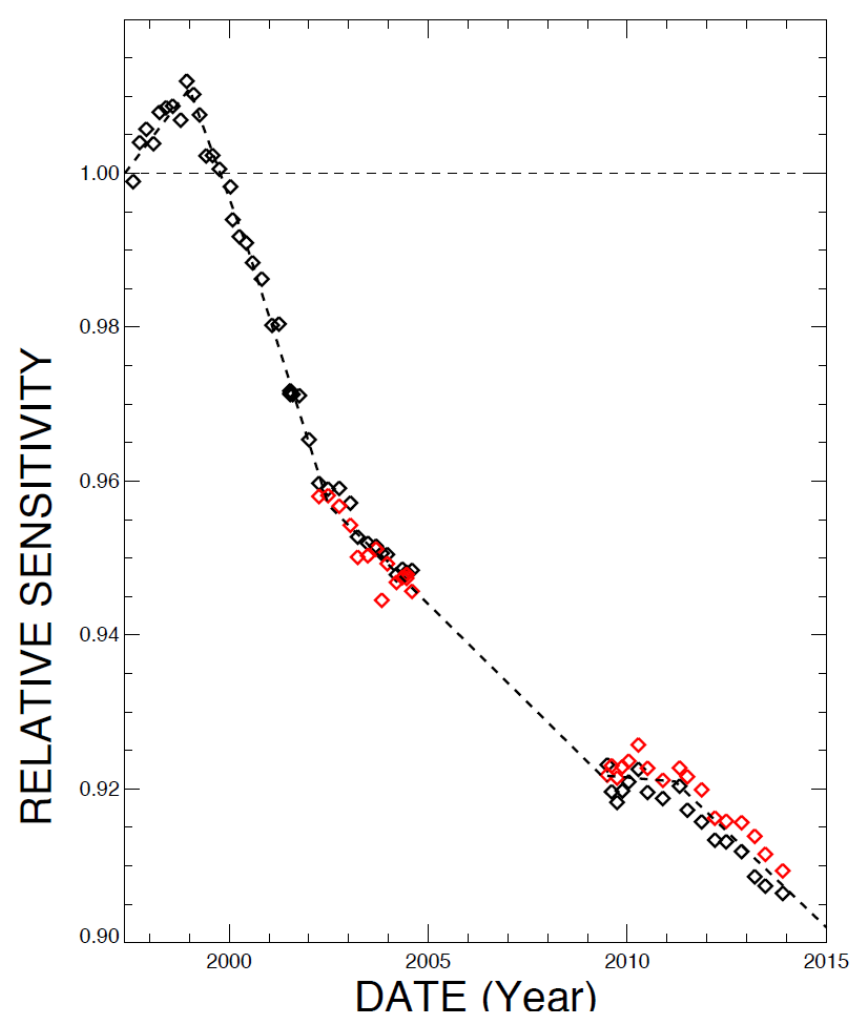

Fig. 9 The time-behaviour of the responsivity of the Space Telescope Imaging Spectrograph (STIS) during observations in space as observed by monitoring a standard star. The black diamonds are data - relating to the wavelength range $200 \mathrm{~nm}$ to $300 \mathrm{~nm}$ and corrected for charge transfer efficiency losses - from the centre of the CCD. The red diamonds correspond to measurements taken at a position near the read-out amplifier. The five-year gap in the data corresponds to the waiting time until STIS could be repaired during SM4. (After Figure 1 in Bohlin et al (2014), with permission.)

physics department of the University of Wisconsin with electron-energy of $240 \mathrm{MeV}$ generated a spectrum resembling that of a B5-star, i.e., the kind of stars to be calibrated. This largely eliminated problems with scattered light. Bless et al performed complete absolute calibrations before and after the flight. In addition, they had prepared "field calibration units", which provided checks of the rocket instrument's responsivity whenever judged beneficial. Field checks could be made three days before, and the day after the flight.

Bless et al then revised the responsivity of the $O A O-2$ (astro)photometers according to the results of the rocket flight. Figure 10 compares the spectral stellar irradiance of $\eta$ UMa obtained in this way with that of the modern CALSPEC database. The agreement within roughly $\pm 10 \%$ is remarkable, particularly as it also substantiates the original uncertainty estimate.

In 1975, a series of laboratory-pedigreed, extraterrestrial measurements of ultraviolet stellar irradiances that came from groups working in Belgium, the Netherlands, the UK, and the US had been available for a comparison.

Aalders et al (1975) at the University in Groningen had calibrated the Astronomical Netherlands Satellite $(A N S)$ based on a detector standard, namely an EMI phototube that had been maintained at the University of Wisconsin. Their estimated calibration uncertainty was $\pm 30 \%$. 


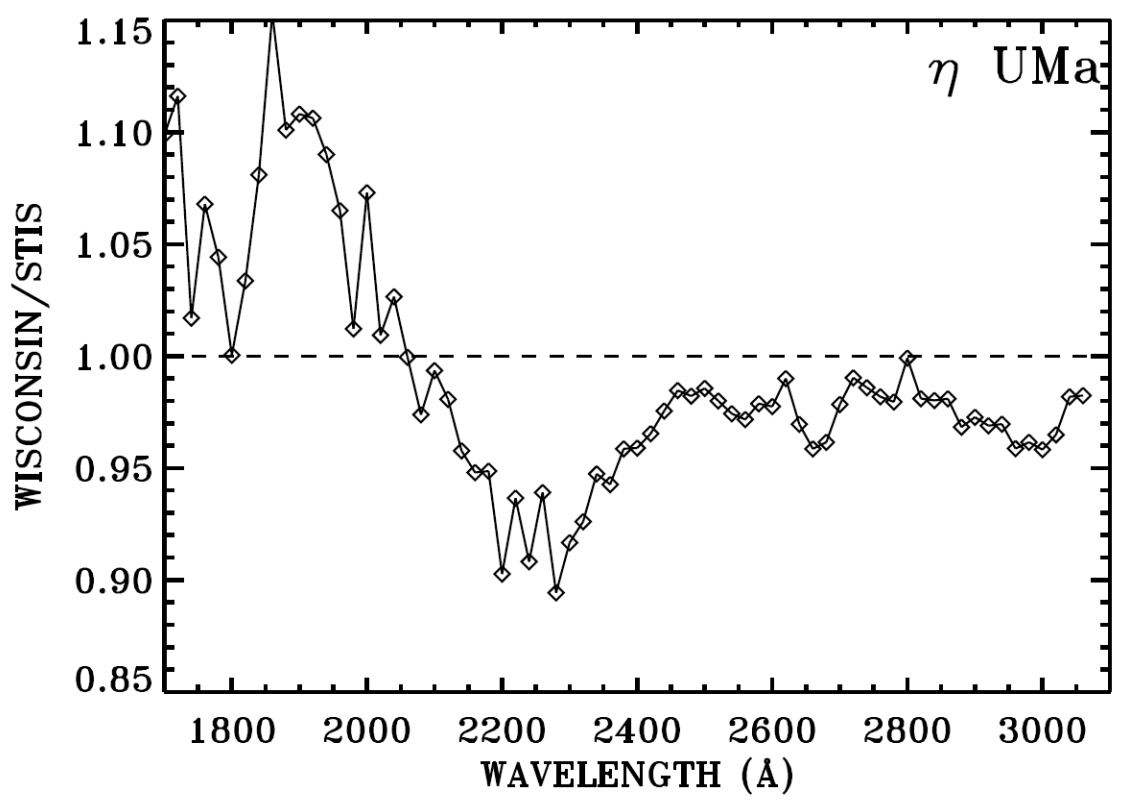

Fig. 10 The spectral stellar irradiance of $\eta$ UMa according to the calibration of $O A O-2$ with a rocket flight carrying an (astro)photometric telescope that had been calibrated by use of synchrotron radiation is compared here with data obtained with HST using the CALSPEC database as calibration file. (After Figure 2 in Bohlin et al (2014), with permission.)

Calibrations of the Sky Survey instrument on ESA's TD-1 satellite by Humphries et al (1976) had been performed independently in Edinburgh and Liège, based on fundamentally different laboratory standards. The uncertainty estimate of the overall calibration was $\pm 20 \%$.

A Johns Hopkins instrument (Henry et al 1975) mounted in the service module of Apollo 1\%, observed low-resolution ultraviolet spectra from lunar orbit. It had been calibrated by use of photodiodes provided by the NBS ${ }^{23}$ The estimate of the calibration uncertainty was $\pm 10 \%$. There was a decline of the responsivity owing to the substantial flux of ultraviolet radiation reflected from the lunar surface, which could, however, be corrected.

At Colorado, Bohlin et al (1974) had measured the spectral irradiance of $\alpha$ Lyr, $\eta$ UMa, and $\zeta$ Oph in the wavelength range $170 \mathrm{~nm}$ to $340 \mathrm{~nm}$ by a rocket flight in 1972 . The standards used in this case were a tungsten standard lamp and two caesium-telluride photodiodes, both calibrated by the NBS. Strongylis and Bohlin (1979) later revisited and corrected these results, and estimated that the uncertainties of the corrected results ranged from $+27 \% /-17 \%$ at $175 \mathrm{~nm}$ to $+15 \% /-12 \%$ at $335 \mathrm{~nm}$.

The four graphs of Figure 11 show ratios between the absolute ultraviolet irradiances of stars measured by the four experiments described above and the corresponding ones obtained by OAO-2. Taken together, these early measurements indicate that the uncertainty estimates were realistic, with one exception, but, unfortunately, still rather sizeable.

This discouraging situation began to improve after observers had studied white dwarfs with IUE. Greenstein and Oke (1979) recognised from studies with the IUE spectroscopic telescope that effective temperatures of degenerate stars - determined by fitting line profiles of the Ly- $\alpha$ absorption - matched those obtained by (astro)photometry in the visible, and also those obtained from the $A N S$ in the ultraviolet, rather well. They thus concluded that an adaptation of the

23 The U.S. National Bureau of Standards, now called National Institute of Standards and Technology (NIST). 


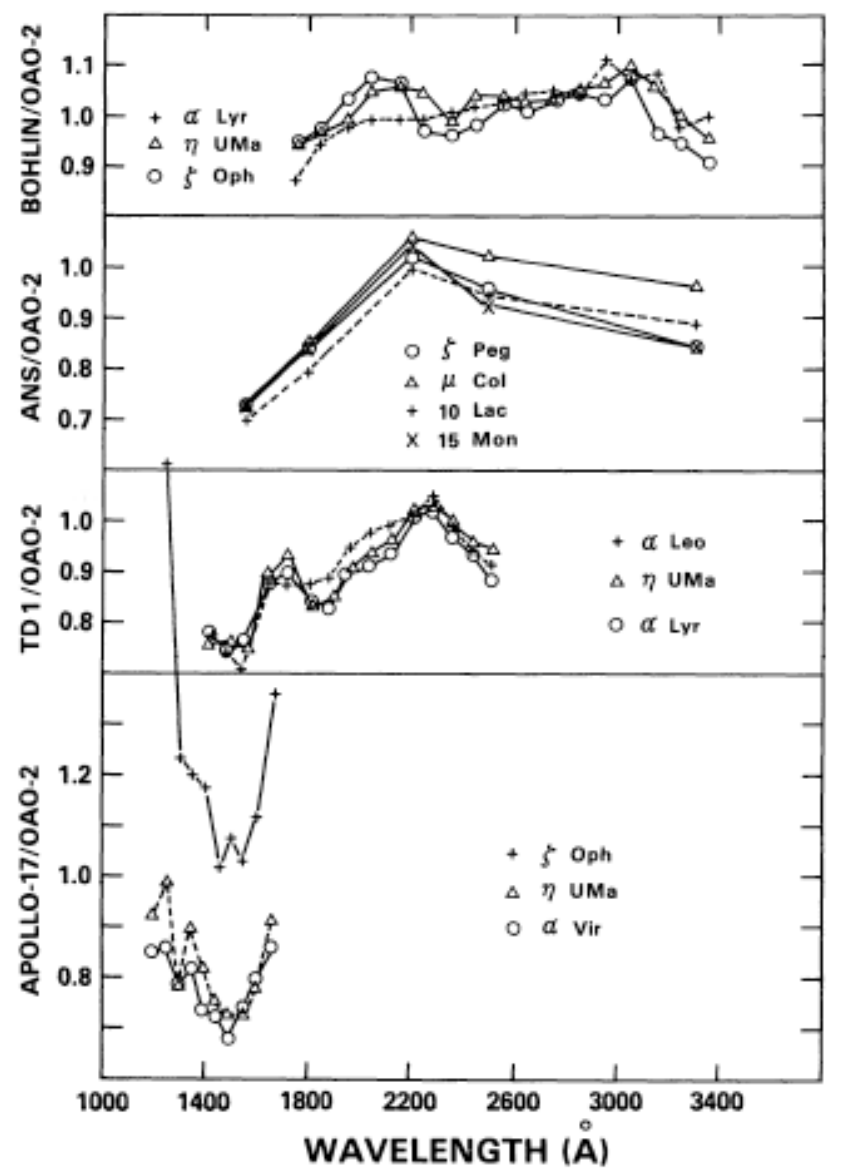

Fig. 11 A comparison of absolute ultraviolet irradiances of four independent space telescopes with those derived from the OAO-2 satellite (Bless et al 1976). Bohlin: Strongylis and Bohlin (1979); ANS: Aalders et al (1975); TD-1: Humphries et al (1976); Apollo-17: Henry et al (1975). Note that the estimated uncertainties of the various measurements, with the exception of those by Henry et al turned out to be realistic. Note however, that the latter had singled out the data points relating to $\zeta$ Oph as being particularly uncertain. (After Figure 4 in Strongylis and Bohlin (1979), with permission.)

IUE calibration was needed. They presaged, "If we accept that theoretical predictions should be correct, corrections to the absolute IUE calibration derived are an upward shift of (3 to 5) \%, with irregular residuals attaining $\pm 7 \%$ '24 Finley et al (1984) later proposed a change of the responsivity of $I U E$ by about ten per cent, after they, too, had found that effective temperatures of white dwarfs obtained from visual magnitude measurements and ultraviolet irradiances measured by IUE were incompatible. Holberg et al (1986) confirmed these earlier conclusions. They had determined temperatures of the atmospheres of white dwarfs by fitting Ly- $\alpha$ profiles obtained from $I U E$ observations and derived from them a correction of the spectroradiometric calibration of IUE. Following a further analysis of the spectra of seven hot DA white dwarfs, Finley et al (1990) suggested that white-dwarf calibration should serve as the calibration for IUE (and HST).

24 The idea that "if we accept that theoretical predictions should be correct ..." was later followed, and has been guiding the calibration of $I U E$ and $H S T$ up to today. Improvements of the models (improved gravity values, non-LTE calculations, for example), which have taken place in the mean time will be mentioned below. 
They thus affirmed Greenstein and Oke's 1979 "conjecture", namely that if one accepted "that theoretical predictions should be correct", this would be an expedient for the absolute calibration of $I U E$.

In preparation for the launch of $H S T$, Bohlin et al (1990) presented 37 "standard stars", whose absolute ultraviolet spectral irradiance distributions for the wavelength range $115 \mathrm{~nm}$ to $330 \mathrm{~nm}$ had been derived by use of the latest in-orbit calibration of $I U E$. There was agreement with the $O A O-2, T D-1$ and $A N S$ data to a few per cent for stars in common. The transition to groundbased data near the atmospheric cut-off between $325 \mathrm{~nm}$ and $340 \mathrm{~nm}$ (Hayes and Latham 1975. Oke and Gunn 1983) implied an uncertainty of about $3 \%$. These 37 standard stars, which had an appropriate distribution across the sky, a range of $10^{4}$ in brightness and a good coverage of spectral types, defined the first ultraviolet calibration of HST. At the time, a comparison with white-dwarf model atmospheres indicated an agreement of about $10 \%$ to $15 \%$.

At the 1993 HST Calibration Workshop, which took place shortly before the originally intended imaging performance of $H S T$ was to be restored during HST Servicing Mission 1, a consensus had developed that one should set the radiometric calibrations of IUE and HST onto the same scale, and also that spectra of further white dwarfs should be obtained for the purpose of improving the absolute calibration with the HST Faint Object Spectrograph (Blades and Osmer 1994).

Bohlin et al (1995) then performed such observations on three additional white dwarfs with the Faint Object Spectrograph of HST, and showed that the spectral irradiance of these three newly observed stars agreed with the model spectra within about $2 \%$. As the (astro)photometry in the blue and visual agreed within better than $1 \%$ in the average as well, these authors recommended that four white dwarfs be primary reference standard stars, and recommended their use for all ultraviolet and optical absolute calibrations over the wavelength range from $100 \mathrm{~nm}$ to $1 \mu \mathrm{m}$ The standard stars in question are G191-B2B, GD 71, GD 153 and HZ 43, whereby ground-based observers should note that HZ 43 has a red companion about $3^{\prime \prime}$ away.

In 1997, Kruk et al published reassuring results: they had observed the hot dwarf star G191-B2B with the Hopkins Ultraviolet Telescope during the Astro-1 space shuttle mission in 1990. HUT's postflight calibration was based on synchrotron radiation and NBS photodiodes as laboratory standards, and showed that the synthetic spectrum of G191-B2B and the effective area of HUT gave results that were consistent within the laboratory measurement uncertainties. Kruk et al thus concluded, "These results validate the use of white-dwarf models as absolute flux standards in the far-ultraviolet, especially for wavelengths shortward of Ly- $\alpha$, where previous experiments often disagreed by large factors."

The calibration for the Astro-2 mission of HUT confirmed these findings: synthetic spectra based on model parameters derived from fits to ground-based spectra - differed from the observations interpreted by use of the laboratory calibration by less than $3 \%$ at all wavelengths (except at the cores of the Lyman lines). In a consistency check, the spectrum of an additional star (BD $+75^{\circ} 325$ ) measured by HUT was found to differ from that observed by HST's Faint Object Spectrograph by at most $5 \%$ in the wavelength region covered by both observations. Kruk et al (1999) therefore declared that their results being "consistent within the laboratory measurement uncertainties, demonstrate that pure hydrogen white dwarfs ... may be used as primary flux standards down to the galactic Lyman edge."

Another experimental confirmation of the white-dwarf calibration quality has been reported by Snow et al (2013): the Solar Stellar Irradiance Comparison Experiment (SOLSTICE) on the $S O R C E$ (Solar Radiation and Climate Experiment) spacecraft is an instrument (McClintock et al 2005a) that is aimed at both solar and stellar irradiance measurements. It observes the Sun and

25 This recommendation, with an extension into the infrared, was made in view of the STIS, which covered the wavelength range $115 \mathrm{~nm}$ to $1 \mu \mathrm{m}$ and was eventually installed on board HST during SM 2 in 1997 . The data for the longer wavelengths were based on observations by Oke (1990) with the $5.1 \mathrm{~m}$ Hale telescope on Palomar Mountain that were later slightly corrected by use of HST FOS observations (Colina and Bohlin 1994). 
stars by the same optics and detectors by changing the size of the entrance aperture and of the exit slit. SOLSTICE was calibrated over the wavelength range $115 \mathrm{~nm}$ to $320 \mathrm{~nm}$ on the SURF-III storage ring with an uncertainty of about $3 \%$ and transferred this laboratory calibration in an early on-orbit observing campaign to 18 stars, before any observations of the Sun would initiate a degradation of the responsivity (McClintock et al 2005b). Figure 12 shows a comparison of absolute irradiances measured by SOLSTICE for three common stars in the CALSPEC database, and again supports both the white-dwarf calibration procedure and the realistic uncertainty estimate of the SOLSTICE laboratory calibration.

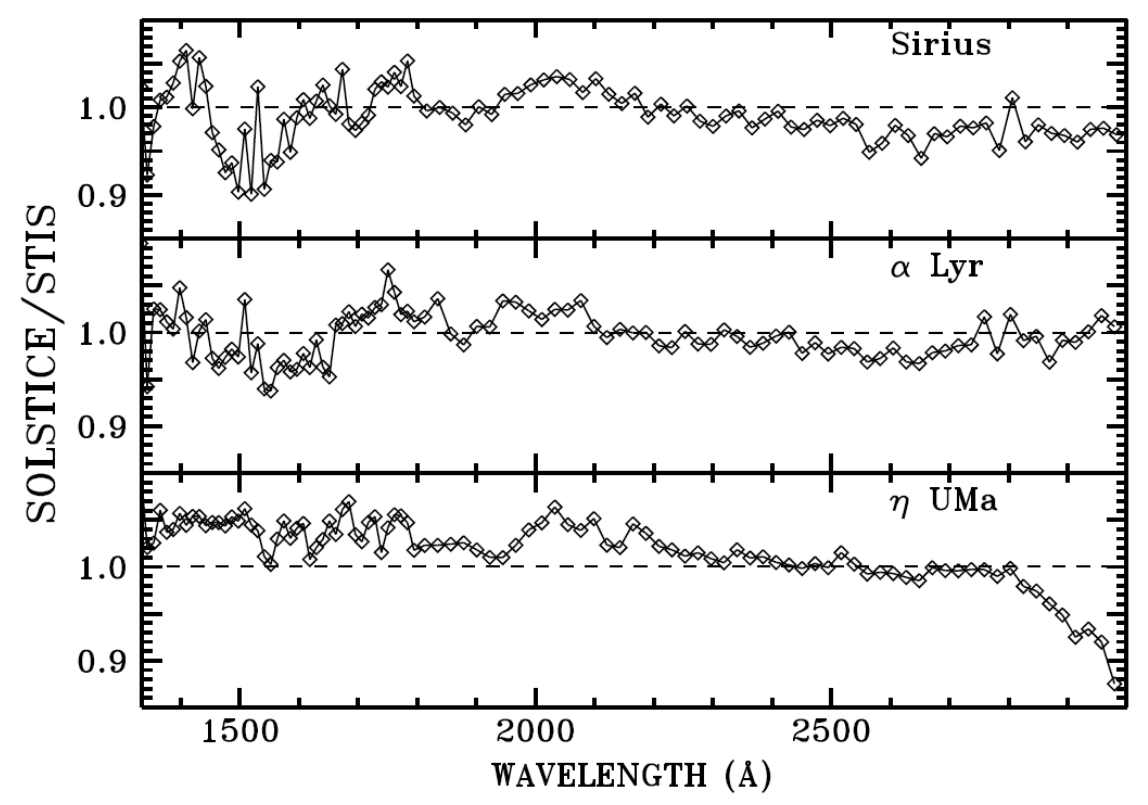

Fig. 12 Comparison of SOLSTICE irradiances for three stars with CALSPEC baseline data (STIS/HST irradiances for $\lambda>170 \mathrm{~nm}$ and $I U E$ irradiances at shorter wavelengths) demonstrates that the values of the synchrotron-based irradiances of SOLSTICE and those of STIS rarely differ by more than $\pm 6 \%$, i.e., $2 \sigma$ of the uncertainty of the laboratory calibration of SOLSTICE. (After Figure 6 in Bohlin et al (2014), with permission.)

In the above text we have addressed the ultraviolet wavelength range in detail, because it was the first domain to be spectroradiometrically calibrated and also because it may be the experimentally most demanding area, when one tries to calibrate the responsivity of traditional space telescopes, i.e., telescopes giving access to the ultraviolet, visible, and infrared ranges of the electromagnetic spectrum. The calibration of vacuum-ultraviolet instrumentation was initially also the most uncertain, not the least because contamination of the surfaces of optical elements and detectors was (and actually still is) difficult to control. The visible part of the spectrum and its extension to ca. $1 \mathrm{\mu m}$ will soon have a more accurate calibration when data from the NIST TCF and from the ACCESS will become available. As mentioned in the introductory paragraph, spectroradiometry in the infrared out to $35 \mu \mathrm{m}$ seems to be settled at an uncertainty level for the irradiance of standard stars around two to three per cent thanks to the untiring efforts of the community (Rieke et al 2008) and particularly the series of 14 papers initiated by Martin Cohen (Cohen et al 1992a) ${ }^{26}$ Realistically, however, the physics-related measurements on the Midcourse

26 Note that the Astrophysics Data System ADS gives access to all papers in a series, whenever one calls up one of the individual papers. 
Space Experiment reported in the last three papers of the series (Price et al 2004 Engelke et al 2006, 2010) tied down the calibration scale to the two-per-cent uncertainty level.

Rather than describing the extended efforts that went into modelling stellar atmospheres, particularly those of white dwarfs with hydrogen atmospheres - exploiting non-LTE effects, determining effective temperatures of stellar atmospheres from line-profile analysis, and deriving stellar gravity via stellar-diameter measurements - we refer to Bohlin et al (2014).

The outcome of these efforts is the CALSPEC archive (Bohlin 2014), which presents a standard calibration scale for use with HST and future JWST observations and which reaches from the vacuum ultraviolet far into the infrared. The calibration scale is backed up by an extensive modelling effort. The spectrum of Sirius calculated by Kurucz $(1979,1993,2013 \sqrt{27}$ for a model with $T_{\text {eff }}=9850 \mathrm{~K}, \log g=4.3$ and an abundance ratio $[\mathrm{M} / \mathrm{H}]=+0.4$ agrees extremely well with the spectrum of Sirius as shown by Bohlin. Remarkably, because the spectrum was calculated long before CALSPEC was established, the original 1993 Kurucz model agrees with the 2013 update within better than $1 \%$ longward of $180 \mathrm{~nm}$. At shorter wavelengths, the update removes some emission lines and, as a consequence, now also fits the observed irradiance significantly better.

As a reminder of the difficulty of radiometric calibration, we may recall in conclusion the optimistic view that Bless et al expressed in 1967, "It appears likely that within the next few years the absolute energy distribution of stars will be as well known in the ultraviolet as in the visual region of the spectrum." Although the path to knowing the absolute energy distribution in the visible on the $0.5 \%$ or at least $1 \%$ level is still ahead of us, the way to a somewhat satisfactory knowledge of the ultraviolet and infrared calibration was much longer, and not principally guided by laboratory measurements. Until a calibration based exclusively on laboratory standards is available, we must remember that the calibration of space telescopes is still resting to a considerable amount on the use of stellar model atmospheres, and, as observations are often supposed to test new models, we must be aware that one has arrived at the hitherto derived calibrations by what, in effect, is a giant bootstrap procedure. This inevitably will lead to testing of models by models, which may lead to circular conclusions.

\section{Examples of calibrated instruments aboard spacecraft}

\subsection{X-ray instruments}

High-resolution X-ray spectroscopy of cosmic sources is the key to understanding physical processes in hot astrophysical plasmas: it provides the essential tools for determining temperatures, densities, element abundances and ionisation stages, and flow and turbulent velocities. Concomitant studies of absorption features provide information about line-of-sight interstellar gas and dust. The absolute radiometric responsivities of X-ray telescopes and spectrometers cannot be unambiguously determined by use of celestial standards because absorption by interstellar gas confounds comparisons with model spectra, because the models themselves are compromised by trace amounts of helium and metals in the real atmospheres, and, finally, and perhaps most fundamentally, because most astronomical sources of EUV and X-rays are variable.

As a consequence of such complications, pre-launch spectroradiometric responsivity characterisation and effective prevention of contamination of the optical elements of the flight models are essential for astronomical EUV and X-ray missions. The accuracy of such determinations directly limits the astrophysical analyses made from them: in order to derive the differential emission measure or the elemental abundances and ionisation stages, relative line and continuum intensities must be known to within a few percent. As a further example, the studies of the Sunyaev-Zel'dovich effect require $\pm 1 \%$ radiometric accuracy. Such constraints drive the calibration requirements.

27 cf., http://kurucz.harvard.edu/stars/ 
Instruments are designed to image far-away sources through the entire entrance aperture. Thus, ideally, spectroradiometric calibrations of telescope-spectrometer combinations would involve irradiation of the full telescope mirror with a parallel beam of radiation with known spectral content. In the absence of an appropriate X-ray collimator this is difficult to achieve in practice, and leads to a finite source-to-telescope distance in the laboratory with the corresponding geometries. In general, the calibration is performed at long-beam X-ray facilities, i.e., with a limited source distance, and the on-ground calibration has to be extrapolated to in-orbit conditions.

Moreover, after launch, the radiometric calibration of high-energy astronomy missions quite often differs from that measured on the ground - not unlike the situation found in other wavelength ranges. As a rule, further changes then occur in the course of the mission, as frequently observed during science operations. In 2006, therefore, an International Astronomical Consortium for High Energy Calibration (IACHEC) ${ }^{28}$ was founded with the aim of harmonising the calibration of high-energy astrophysics missions. This forum is endorsed and led by the user group of the two major missions, XMM-Newton and Chandra, and is funded by the experimenters of participating missions. In the framework of IACHEC, a sustained effort is undertaken to inter-calibrate highenergy missions by use of a set of "standard candles" and, in this way, to minimise calibration uncertainties (Sembay et al 2010). In the following, we will trace the development of instrumentation and calibration methods in the course of time by describing some example missions with their calibration measures and status.

Uhuru (1970 to 1973; Giacconi et al 1971) was the first satellite launched specifically for the purpose of X-ray astronomy, using two sets of proportional counters and observing in the range from $2 \mathrm{keV}$ to $20 \mathrm{keV}$. Information on this and other previous missions can be found on the webpage of the High Energy Astrophysics Science Archive Research Center (HEASARC) which is the primary archive of NASA's (and other space agencies') missions studying electromagnetic radiation from extremely energetic cosmic phenomena ${ }^{29}$ In particular its database stores information (such as targets and sequences) and files for calibration and cross-calibration of high-energy astronomical instrumentation 30

ESA's EXOSAT (1983 to 1986; Taylor et al 1981 ${ }^{31}$ covered the energy range between $0.05 \mathrm{keV}$ and $50 \mathrm{keV}$. It was followed by the ROSAT mission (Röntgensatellit, 1990 to 1999; Aschenbach et al 1981), carrying a German-built imaging X-ray Telescope (XRT) with three focal-plane instruments, two Position Sensitive Proportional Counters (PSPC), and the US-supplied High Resolution Imager (HRI). The X-ray mirror assembly was a grazing-incidence four-fold nested Wolter I telescope with an $84 \mathrm{~cm}$ diameter aperture and $240 \mathrm{~cm}$ focal length. The XRT assembly was sensitive to X-rays between $0.1 \mathrm{keV}$ and $2 \mathrm{keV}$. In addition, a British-supplied extreme ultraviolet telescope, the Wide Field Camera (WFC), was coaligned with the XRT and covered the energy band from $0.042 \mathrm{keV}$ to $0.21 \mathrm{keV}$. Calibration had, to a large part, been performed at the PANTER facility ${ }^{32}$ of the MaxPlanck-Institut für extraterrestrische Physik (MPE) in Garching (Freyberg et al 2005), documents are available at the websites of the MPE ${ }^{33}$ and HEASARC ${ }^{34}$ The ROSAT Users' Handbook (Briel et al $1997{ }^{35}$ contains additional, more detailed information.

\footnotetext{
28 Updated general information on IACHEC is available on the web site http://web.mit.edu/iachec/. Results of the IACHEC collaboration are published as refereed papers and made accessible through http://web.mit.edu/iachec/ papers/

29 http://heasarc.gsfc.nasa.gov/

30 http://heasarc.gsfc.nasa.gov/docs/heasarc/caldb/caldb_intro.html and http://heasarc.gsfc.nasa.gov/docs/heasarc/ caldb/caldb_xcal.html

31 http://www.cosmos.esa.int/web/exosat/home

32 http://www.mpe.mpg.de/heg/panter

33 http://www.mpe.mpg.de/xray/wave/rosat/doc/calibration/index.php

34 http://heasarc.gsfc.nasa.gov/docs/heasarc/caldb/caldb_docs_rosat.html

35 http://www.mpe.mpg.de/xray/wave/rosat/doc/ruh/rosathandbook.php
} 
The Italian-Dutch BeppoSAX mission (1996 to 2003) comprised five instruments to cover the range from $0.1 \mathrm{keV}$ to $300 \mathrm{keV}$, four X-ray telescopes working in conjunction with one of the following detectors: the Low Energy Concentrator Spectrometer (LECS), the Medium Energy Concentrator Spectrometer (MECS), the High Pressure Gas Scintillator Proportional Counter (HPGSPC), and the Phoswich Detection System (PDS), as well as two units of a Wide Field Camera (WFC) ${ }^{36}$ Ground calibration of the LECS and MECS had been done at beamlines of the BESSY and PANTER facilities, component by component, and at the instrument level (Parmar et al 1997, Boella et al 1997). The HPGSPC and the PDS had been calibrated by use of radioactive sources; the PDS included a movable calibration source Co ${ }^{57}$ (Santangelo et al 1997). The Crab nebula and supernova remnants were used for flux cross-calibration in orbit ${ }^{37}$

The Rossi X-ray Timing Explorer, RXTE (1995 to 2012; Bradt et al 1993; Rothschild et al 1998) measured in the energy range between $2 \mathrm{keV}$ and $250 \mathrm{keV}$, by use of a Proportional Counter Array (PCA), the High Energy X-ray Timing Experiment (HEXTE), and an All-Sky Monitor (ASM). The HEXTE consisted of two clusters of four phoswich scintillation detectors, fast switching between background and targets with a sampling time of eight microseconds. Automatic gain control was provided by comparison with a radioactive source $\left({ }^{241} \mathrm{Am}\right)$ mounted in each detector's field of view.

Suzaku (formerly ASTRO-EII) is a Japanese X-ray astronomy satellite launched in 2005. It is carrying high-resolution, wide-band instruments for detecting signals ranging from soft X-rays up to gamma-rays $(0.3 \mathrm{keV}$ to $600 \mathrm{keV}){ }^{38}$ The payload systems are the X-ray Spectrometer (XRS), an X-ray calorimeter, the X-ray Telescope (XRT), the X-ray Imaging Spectrometer (XIS), and the Hard X-ray Detector (HXD). The X-ray calorimeter of Suzaku was the first such instrument to be flown in space and unfortunately stopped operation soon after launch due to failure of the cooling system. Description and database of the calibration are given at the webpages ${ }^{39}$

The X-ray Multi-Mirror Mission (XMM-Newton; Jansen et al 2001) was launched in 1999 as the second "cornerstone" of ESA's Horizons 2000 Programme (European Space Agency 1994). This space observatory performs high-throughput spectroscopy of cosmic X-ray sources. Its effective areas - over nearly its entire spectral domain, $0.1 \mathrm{keV}$ to $10 \mathrm{keV}(12.5 \mathrm{~nm}$ to $0.125 \mathrm{~nm})-$ are approximately $1000 \mathrm{~cm}^{2}$ and $100 \mathrm{~cm}^{2}$, respectively, for spectrometric imaging and high-resolution spectroscopy. XMM-Newton was calibrated by a combination of the three calibration methods, $(i)$ through (iii), enumerated in Section 2.4. There were pre-flight calibrations at the component and sub-system level and in end-to-end configurations. Specifically, the three telescope assemblies, each consisting of 58 Wolter type-I mirrors in a nested co-axial and confocal configuration, were tested in the PANTER facility of the MPE. Comprehensive numerical modelling was used to generate a calibration database from which the laboratory tests, taken with a finite source distance, could be extrapolated to in-orbit conditions (Gondoin et al 1996, 1998). The spectroradiometric calibration of XMM-Newton is monitored in orbit by regularly observing a selected set of sources and crosscalibration is carried out with contemporary X-ray observatories.

All three telescope assemblies have CCD arrays - the European Photon Imaging Cameras (EPIC) - in their focal plane. Two of them are equipped with MOS-CCDs (Turner et al 2001), and one uses pn-CCDs (Strüder et al 2001). These cameras had also been calibrated in several steps. First, their quantum efficiencies were determined by use of synchrotron light sources. Then, the effective area of the telescope-camera combination was calibrated in the PANTER facility. However, to minimise contamination of the flight mirrors, the end-to-end calibrations were carried out with the qualification-model rather than with the actual flight-model mirror assemblies.

\footnotetext{
36 Additionally, it carried a Gamma-Ray Burst Monitor (GRBM) of four CsI(Na) scintillators that were also used as active lateral shields of the PDS experiment. Its calibration is described by Amati et al (1997).

37 http://www.asdc.asi.it/bepposax/calibration.html https://heasarc.gsfc.nasa.gov/docs/sax/sax.html

38 http://www.astro.isas.jaxa.jp/suzaku/

39 http://www.astro.isas.ac.jp/suzaku/caldb/, http://www.astro.isas.jaxa.jp/suzaku/process/caveats/
} 
The two Reflection Grating Spectrometers on board XMM-Newton (den Herder et al 2001) are objective spectrometers, i.e., they have no slits. Each of them collects radiation from half the aperture of the two telescope assemblies that feed the MOS-CCDs. The third telescope assembly is unobstructed by gratings and feeds the pn-CCD camera. Each spectrometer uses an array of 182 plane gratings with variable ruling-frequency. Their calibration was performed in three stages. First, the individual gratings and the CCD detectors were characterised. Then measurements were made of the assemblies of the two reflection grating arrays with their focal-plane cameras. Finally, the effective area was determined - again in the PANTER facility - in end-to-end tests. With the finite source distance, the mirror assemblies and the reflection-grating array were under-illuminated, and, consequently, the measured effective area had to be corrected by use of ray-tracing calculations. The results on the overall effective area (measured on axis at six energies, but at one energy only off axis) agreed with the model calculations to within $5 \%$ in the first and second grating order (den Herder et al 2001). XMM-Newton also carries internal X-ray sources $\left({ }^{244} \mathrm{Cm}\right)$. Although these do not permit an end-to-end calibration in orbit, they are useful for monitoring changes in the stability of the instrument responsivities. In particular they can be used for absolute energy calibrations, to establish the system gain at the time, and under the conditions of, a given observation. In principle, the internal sources also permit the measurement of radiation damage in orbit, manifesting itself in a loss in charge-transfer efficiency. However, the damage monitoring turned out to be considerably more complicated in the $X M M$ case because the calibration source is also cool and thus a sink for contaminants.

An overview of the recent calibration status is given by de Vries et al $(2015)$ and gives an uncertainty of $10 \%$ for the effective area in the energy range $0.5 \mathrm{keV}$ to $1 \mathrm{keV}$, somewhat larger for higher-energy values, and up to $40 \%$ at the low-energy end. Water vapour contamination, resulting in a stable oxygen edge in the instrument response, has been observed, most probably due to the long period between purging the instrument, sealing, and the launch. Additionally, loss of effective area at longer wavelengths increasing over time, attributed to out-gassing of hydrocarbons by the carbon-fiber-reinforced structures of the telescope tube, has been found.

Cross-calibration between the different XMM-Newton instruments and between XMM-Newton and various other observatories such as Chandra, Suzaku, Swift, BeppoSAX, RXTE, are being performed on a regular basis (see, e.g., the $X M M$ calibration portal and the documents found there) ${ }^{40}$ Simultaneous observations of a regular X-ray burster, GS 1826-238, with Chandra and $R X T E$ resulted in agreement of the fluxes measured by PCA/RXTE and Chandra within their formal uncertainties; XMM-Newton's EPIC-pn measured (14.0 \pm 0.3$) \%$ less flux than the PCA/RXTE (Güver et al 2015).

The Chandra X-ray Observatory (CXO, launched 1999; Weisskopf et al 2002), formerly known as the Advanced X-ray Astrophysics Facility $A X A F$, carries a High Resolution Mirror Assembly (HRMA), two focal-plane instruments, the High Resolution Camera (HRC) and the Advanced CCD Imaging Spectrometer (ACIS), and two sets of transmission gratings, the High Energy Transmission Grating (HETG) and the Low Energy Transmission Grating (LETG). The gratings can be moved in and out of the optical paths to both detectors, permitting both spectroscopy and imaging to be performed. The combination of high resolution, large collecting area, and sensitivity to higher-energy X-rays makes it possible for Chandra to study extremely faint sources in crowded fields whose lower-energy radiation is sometimes strongly absorbed. Since transmission - away from absorption edges - increases with increasing energy, higher-energy X-rays are less affected by obscuring material so that the irradiance of the sources can be determined. At the same time the hypothesis that some of these sources may be "standard candles" can be tested. And, if such "standard candles" are found and calibrated, distances to nearby galaxies can be determined. These distances are a crucial step in the derivation of the Hubble Constant.

40 http://xmm2.esac.esa.int/external/xmm_sw_cal/calib/ 
Chandra has been calibrated at the X-ray and Cryogenics Facility (XRCF) 41 at the NASA Marshall Space Flight Center. The determination of the effective areas was a major undertaking. In order to meet its science objectives, the $C X O$ project started with a calibration goal of determining the effective areas of the various combinations of telescope mirror and focal-plane science instruments with overall uncertainties at the $\pm 1 \%$ level (Kellogg et al 1997) through an extensive programme of sub-system and end-to-end measurements and modeling similar to that discussed above for XMM. The effective area of the HRMA is given by Schwartz et al (2000); that for the ACIS and HRC focal plane detectors is given by Bautz et al (2000) and Murray et al (2000), respectively. The website provides links to references and the calibration status, as well as to the most recent calibration workshops ${ }^{42}$

The NASA Small-Explorer (SMEX) mission NuSTAR (Nuclear Spectroscopic Telescope Array, launched in 2012, Harrison et al 2013 ${ }^{43}$ is, for the first time, using focusing optics in the highenergy X-ray domain (3 keV to $79 \mathrm{keV}$ ) instead of coded apertures. It consists of two co-aligned grazing-incidence telescopes (of ten metre focal length) with specially coated optics and newly developed (hybrid pixel) detectors. For the ground calibration of the optics, the Rainwater Memorial Calibration Facility (RaMCaF) has been built, providing a long $(175 \mathrm{~m})$ high-energy beamline in order to approximate the situation in space (Brejnholt et al 2011, 2012). In-flight calibration has largely been using the Crab nebula (Madsen et al 2014, and references therein).

\subsection{EUV and FUV instruments}

Although primarily designed for observation of the atmospheres of solar system planets, the Voyager spacecraft (launched 1977; Broadfoot et al 1977) made many of the first observations of hot stars at EUV wavelengths. Spectroradiometric accuracy was a major goal of this work, but uncertainties in calibrations - based on sounding rocket observations - plagued the results (see Holberg et al 1991, and discussion therein).

Before the Hopkins Ultraviolet Telescope HUT (operated on two Space Shuttle missions in December 1990 and March 1995, cf., Section 3), however, there were no other useful spectroradiometric observations at these wavelengths from spacecraft. The calibrations of the ORFEUS I and II mission ${ }^{44}$, for example, were not based on direct calibration but on comparison to modelled spectra of G191-B2B and HZ 43, respectively (Hurwitz et al 1998 Barnstedt et al 1999). A comparison of fluxes from targets common to both ORFEUS missions indicated that the calibration of the Berkeley spectrometers differed by about $10 \%$ (Hurwitz et al 1998). The HUT observations (Kruk et al 1997: Dixon et al 2013) showed that the final Voyager calibration was accurate to $\pm 10 \%$ over the wavelength ranges $91 \mathrm{~nm}$ to $118 \mathrm{~nm}$.

The performance of the Extreme Ultraviolet Explorer (EUVE ${ }^{45} 1992$ to 2001; Bowyer and Malina 1991), which comprised four grazing-incidence telescopes with a number of thin, metal-film filters that defined six, partially overlapping, passbands between $6 \mathrm{~nm}$ and $75 \mathrm{~nm}$, is discussed by Sirk et al (1997). The pre-launch effective area of EUVE had been determined from laboratory measurements at discrete wavelengths by use of a transfer-standard photodiode. Effective-area values between the calibration points were modelled from measured values of mirror reflectivities, filter transmission, and detector responsivity. The differences between the measured and modelled

\footnotetext{
41 https://optics.msfc.nasa.gov/

42 http://cxc.harvard.edu/

43 http://www.nustar.caltech.edu/

44 Orbiting Retrievable Far and Extreme Ultraviolet Spectrometers, 1993 and 1996 on the Astro-SPAS, a reusable shuttle-launched space platform (Grewing et al 1998)

45 http://www.ssl.berkeley.edu/euve/index.html
} 
effective areas were about $\pm 30 \%$. The EUVE data are archived at HEASARC and MAST 46 Inorbit calibration using WD models HZ 43 and GD 153 (Sing et al 2002) has been shown to be consistent with $10 \%$ uncertainties except at the longest wavelengths $(\lambda>60 \mathrm{~nm})$.

The calibration of the Far Ultraviolet Spectroscopic Explorer (FUSE, 1999 to 2007, Sahnow et al 2000b) is based on WD models only (Sahnow et al 2000a). Originally, uncertainties had been calculated to be less than $10 \%$, however, repeated observations of standard white-dwarf stars showed that, while the instrument's responsivity had been roughly constant for the first two years of the mission, it later declined at a rate of $5 \%$ to $10 \%$ per year, (see also Conard et al 2000) ${ }^{47}$

\subsection{VUV and UV instruments}

The International Ultraviolet Explorer (IUE, 1978 to 1996; Boggess et al 1978) was a common project of NASA, the UK Science Research Council and the European Space Agency (ESA). As mentioned in Section 3, all archived data were recalibrated by use of the pure hydrogen WD G191$\mathrm{B} 2 \mathrm{~B}{ }^{48}$ The $I U E$ flux reference deviates by $6 \%$ from the current $H S T$ CALSPEC reference (see Bohlin et al 2014, and references therein).

The Hubble Space Telescope $H S T,{ }^{49}$ a NASA/ESA mission, has been launched in 1990, is still operating as of 2015, and may last until 2020. Its main instruments observe in the ultraviolet, visible, and near infrared. Five subsequent Space Shuttle missions repaired, upgraded, and replaced systems on the telescope, the last mission having taken place in 2009.

The current set of science instruments includes three cameras and two spectrographs. The calibration of $H S T$ has been described in Section 3. An overview of the standards used in the HST CALSPEC procedures is given by Bohlin et al (2014). Electronic versions of the UV spectra of all $H S T$ calibration standards as well as overviews over the instruments and their documentations and the proceedings from the calibration workshops can be found on the STScI Web pages 50

4.4 Visible and near-, mid-, and far-infrared instruments

The absolute calibration of visible and NIR spectrometric telescopes in space, follows a process similar to that used for the calibration of ground-based visible telescopes: A primary celestial standard is used to set the absolute flux scale and a small set of secondary standards is used in the calibration of the different instruments over their corresponding wavelength and responsivity ranges (cf., Section 3).

The infrared astronomy satellite $A K A R I$ (Murakami et al 2007, Yamamura et al 2010, before launch called Astro- $F)^{51}$ has been in operation from 2006 to 2011 . It has been developed by the Japan Aerospace Exploration Agency JAXA, in cooperation with institutes from Europe and Korea. Its primary mission was to survey the entire sky in near-, mid-, and far-infrared, from $1.7 \mu \mathrm{m}$ to $180 \mu \mathrm{m}$, through its $68.5 \mathrm{~cm}$ aperture telescope and with two kinds of instruments: the FIS (Far-Infrared Surveyor) for far-infrared observations and the IRC (InfraRed Camera) for near and mid-infrared observations. The calibration of the $A K A R I$ Far-Infrared Imaging Fourier Transform Spectrometer (FIS-FTS) has been described by Murakami et al (2010). It is based on

46 http://archive.stsci.edu/euve/

47 cf., http://fuse.pha.jhu.edu/analysis/calfuse_wp0.html;http://fuse.pha.jhu.edu/analysis/calfuse_wp1.html

48 IUE data are available from the Mikulski Archive for Space Telescopes (MAST), http://archive.stsci.edu/iue

49 http://www.stsci.edu/hst/ http://www.spacetelescope.org/

50 The two web sites http://www.nasa.gov/mission_pages/hubble/main/index.html and http://www.spacetelescope. org/about/general/instruments/give an in-depth overview of $H S T$, its history and its instruments.

51 http://www.ir.isas.jaxa.jp/ASTRO-F/Outreach/souti_e.html 
bright astronomical sources, such as stars, asteroids and planets. An internal blackbody source has been used for monitoring the detector responsivity over time. The relative uncertainty of the calibration of the continuum has been estimated to be $\pm 20 \%$ or better over the entire wavelengh range, and the absolute uncertainty is estimated to be better than $+40 /-60 \%$. For the IRC, the (astro)photometric accuracies using stars have been found to be better than $20 \%$ (Takita et al 2012, Arimatsu et al 2014).

ESA's Herschel Space Observatory (originally named FIRST, Far InfraRed and Sub-millimetre Telescope; Pilbratt 2008) has been launched in 2009, together with ESA's Planck mission. It has been equipped with the largest - i.e., $3.5 \mathrm{~m}$ diameter - single mirror built so far for a space telescope. Its operational phase ended in 2013 after the helium supply had been exhausted. Herschel's three instruments are the HIFI (Heterodyne Instrument for the Far Infrared), the PACS (Photodetector Array Camera and Spectrometer), and the SPIRE (Spectral and Photometric Imaging Receiver), covering the wavelength range from $55 \mu \mathrm{m}$ to $672 \mu \mathrm{m}$, combining spectrometry, imaging spectrometry, and imaging photometry. For calibration, three sets of celestial standards and associated models have been used: planetary models, stellar models, and asteroid models. Data in the models have been compiled from flyby missions, near-infrared and sub-millimetre ground-based observations, space-based observations, radar measurements, and known planetary atmosphere and stellar atmosphere conditions. The set of asteroids used for calibration fills the gap between the sub-millimetre and millimetre range covered by Mars, Uranus, and Neptune, and the mid-IR range covered by bright calibration stars. The thermophysical model predictions agree within $5 \%$ with the available (and independently calibrated) Herschel measurements (Müller et al 2014).

The HIFI high-resolution heterodyne spectrometer observes in the two bands: $157 \mu \mathrm{m}$ to $212 \mu \mathrm{m}$ and $240 \mu \mathrm{m}$ to $625 \mu \mathrm{m}$. The instrumentation for imaging in the infrared, in particular using superconducting mixers as detectors, has been described for example by Wild (2013) and Swinyard and Wild (2013). HIFI uses internal loads to determine sensitivities for each frequency setting, and very accurate frequencies can be established by the local oscillators. Mainly planets (Mars and Neptune) are used for calibration.

PACS consists of an imaging photometer and a medium-resolution grating spectrometer (Poglits h et al 2010). From a set of "fiducial stars" selected for calibration, five have been used for the final analysis: $\beta$ And, $\alpha$ Cet, $\alpha$ Tau, $\alpha$ Boo, and $\gamma$ Dra (Balog et al 2014). For the special observing mode of "chop-nod" 52 very high accuracy can be reached. The relative (astro)photometric calibration precision (repeatability) has been found to be $1 \%$ in the blue and green bands and up to $5 \%$ in the red band ${ }^{53}$ At this precision, inconsistencies among the stellar calibration models become noticeable, and the absolute calibration accuracy is mainly limited by the model uncertainty, which is $5 \%$ for all three bands (Nielbock et al 2013). For the principal observation mode (scan map, with a boustrophedon pattern) the relative accuracy varies from $0.5 \%$ in the blue band to $2 \%$ in the red. The systematic difference in flux calibration to the chop-nod mode amounts to less than $6 \%$.

The SPIRE instrument is an imaging photometer and imaging Fourier transform spectrometer (FTS, see also Griffin and Ade 2013), both operating over a frequency range of approximately $450 \mathrm{GHz}$ to $1550 \mathrm{GHz}(670 \mu \mathrm{m}$ to $190 \mu \mathrm{m})$. After correction for pointing errors, the absolute flux calibration has been found to be uncertain to less than $6 \%$, and for extended sources where mapping is required to at least $7 \%$, with a precision of about $1 \%$ (Swinyard et al 2014).

Cross-calibration programmes have shown that the agreement between the spectrometers, although based on different prime calibrators, e.g., PACS, using stellar models, and SPIRE, using

\footnotetext{
52 chopping and nodding in perpendicular direction, used to reduce background signal in particular for point-source photometry

53 These bands are centred around the wavelengths $56 \mu \mathrm{m}, 100 \mu \mathrm{m}$ and $160 \mu \mathrm{m}$, respectively. An in-depth description of the filter bandpasses and how they have to be used in comparison with other instruments, can be found on the PACS calibration web sites http://herschel.esac.esa.int/twiki/bin/view/Public/PacsCalibrationWeb.
} 
Uranus and Neptune, is better than $20 \%$. Evaluation of the data as well as update and improvement of the theoretical models is ongoing (Fulton et al 2014, Naylor et al 2014, Müller et al 2014) ${ }^{54}$

\subsection{Infrared and microwave instruments}

Ground-based absolute IR flux measurements with direct reference to laboratory standards, such as those by Selby et al (1983), Blackwell et al (1983), Mountain et al (1985), and Booth et al (1989), have been introduced in Section 3 together with the space-based absolute mid-infrared calibrations by the Midcourse Space Experiment. The latter were carried out by use of emissive reference spheres that were ejected and observed as point sources with calibrations based on laboratory data and basic physics (Cohen et al 2001, Price et al 2004).

Proceeding to the microwave region: studies of the Cosmic Microwave Background (CMB) and its polarisation require extremely high precision (cf., Lamarre and Dole 2013). In this wavelength range the Planck mission (2009 to 2013) ${ }^{55}$ refined the measurements of the CMB radiation of its predecessors COBE (Cosmic Background Explorer, 1989) ${ }_{56}^{56}$ and WMAP (Wilkinson Microwave Anisotropy Probe, 2001) [57 Within the NASA HEASARC web archive, the data center for CMB research, the Legacy Archive for Microwave Background Data Analysis ( $\Lambda$ AMBDA) 58 provides information on the missions and data.

The in-flight calibrations of WMAP and Planck have been done by comparing the raw data to the temperature signal expected from the known dipole anisotropy. This dipole anisotropy is resulting from the motion of the spacecraft relative to the Sun, as well as by motion of the solar system with respect to the CMB rest frame.

Planck carried two scientific instruments: the High Frequency Instrument, or HFI, and the Low Frequency Instrument, or LFI. LFI has been designed to produce high-sensitivity, multi-frequency measurements of the microwave sky in the frequency range of $27 \mathrm{GHz}$ to $77 \mathrm{GHz}$ (wavelength range $11.1 \mathrm{~mm}$ to $3.9 \mathrm{~mm}$ ). The instrument consists of an array of 22 tuned radio receivers located in the focal plane of the telescope. The radiometric calibration is based on the combination of the orbital dipole plus the solar dipole. The solar dipole provides a signal of a few millikelvin with the same spectrum as the CMB anisotropies that is visible throughout the mission. An internal reference load at $4 \mathrm{~K}$ is used for monitoring the calibration stability of the $30 \mathrm{GHz}$ radiometers. Additionally, the brightness temperature of Jupiter is compared. The calibration uncertainties of the $2015 \mathrm{LFI} /$ Planck data release are given as $0.20 \%$ at $70 \mathrm{GHz}, 0.26 \%$ at $44 \mathrm{GHz}$, and $0.35 \%$ at $30 \mathrm{GHz}$. These updated results agree very well with the data from the HFI instrument and within $1 \sigma$ with WMAP results (Planck Collaboration et al 2015b) 59

Similarly, HFI has been designed to produce high-sensitivity, multi-frequency measurements of the diffuse radiation permeating the sky in all directions in the frequency range of $100 \mathrm{GHz}$ to $857 \mathrm{GHz}$ (wavelength range $3.0 \mathrm{~mm}$ to $0.35 \mathrm{~mm}$ ). The instrument consists of an array of 52 bolometric detectors placed in the focal plane of the telescope. The components and subsystems of HFI have been calibrated and tested on the ground, the focal plane units have been characterised in the Saturne cryostat of the calibration facility of the Institut d'Astrophysique Spatiale in Orsay and, after being integrated, on the satellite in a cryogenic vacuum chamber at the CSL (Centre Spatial

\footnotetext{
54 http://www.cosmos.esa.int/web/herschel/home

55 http://www.cosmos.esa.int/web/planck

56 http://lambda.gsfc.nasa.gov/product/cobe

57 http://map.gsfc.nasa.gov

58 http://lambda.gsfc.nasa.gov

59 Preliminary results had indicated some discrepancy to $W M A P$, which could be eliminated by improved data analysis; for updated information see the webpages of the Planck Collaboration http://www.cosmos.esa.int/web/ planck/publications.
} 
de Liège). A preliminary absolute response was estimated during the focal plane calibration with an uncertainty of $10 \%$ (and a relative pixel to pixel calibration of $3 \%$ ). The pre-launch calibration of HFI had been carried out between September 2004 and August 2008 (Pajot et al 2010). The in-flight calibration of the submillimetre channels of HFI relies on the calibration of the FIRAS experiment on the $C O B E$ satellite, which had provided the most accurate radiometric calibration for extended sources in the millimetre and submillimetre wavelength range to date. FIRAS used an absolute black body to provide a flux calibration with an accuracy superior to $1 \%$ below $400 \mathrm{GHz}$ and $3 \%$ above (Mather et al 1999). To obtain the best calibration accuracy over the large range from $100 \mathrm{GHz}$ to $857 \mathrm{GHz}$, two different radiometric calibration schemes have to be used. The higher frequency data are calibrated by use of models of planetary atmospheric emission (Uranus and Neptune). The lower frequencies (from $100 \mathrm{GHz}$ to $353 \mathrm{GHz}$ ) are calibrated by use of the timevariable cosmological microwave background dipole, i.e., the dipole produced by the proper motion of Planck with respect to the rest frame of the CMB. This source of calibration only depends on the satellite velocity with respect to the solar system and permits an independent measurement of the amplitude of the CMB solar dipole, $(3364.5 \pm 0.8) \mu \mathrm{K}$, which is $1 \sigma$ higher than the WMAP measurement with a direction that is consistent between both experiments (Planck Collaboration et al 2015a). Uncertainties in the absolute radiometric calibration have been found to lie in the range from $0.54 \%$ to $10 \%$ from $100 \mathrm{GHz}$ to $857 \mathrm{GHz}$. Details of the subsequently improved postlaunch calibration can be found on the Planck webpages and especially in the publications of the Planck collaboration 60

\subsection{Solar spacecraft}

Solar physicists have striven towards spectroradiometric measurements of the solar spectrum from space since the 1960s. A series of eight Orbiting Solar Observatories $(O S O)$ was launched by NASA between 1962 and 1975. Instrumentation included spectrometers and radiometers for EUV and $\mathrm{X}$-ray measurements. The telescope mirror and the spectrometer of the photoelectric spectroheliometer on $O S O-6$ were separately calibrated before launch, the latter by use of transfer-standard photodiodes (Huber et al 1973). However, spatial non-uniformities in the performance of a concave grating used in the spectrometer calibration to produce a monochromatic beam of test radiation limited the pre-launch radiometric uncertainty of $O S O-6$ to $10 \%$. Such optical non-uniformities also affected the pre-launch calibration of CDS on the Solar and Heliospheric Observatory SOHO and the in-orbit performance of UVCS, also on $\mathrm{SOHO}$; both instruments will be discussed later in this section.

The calibration of the S-055 spectrometer on Skylab was similar to that of the OSO-6 instrument mentioned above (Reeves et al 1977a), and performance was monitored during the mission by means of underflights. Nevertheless, the ultimate S-055 radiometric accuracy was insufficient to disentangle variations in the VUV output of the Sun from changes in the instrument responsivity (cf., Figure 4 ).

\section{Total irradiance monitors}

A number of spaceborne instruments dedicated to total solar irradiance measurements have inorbit radiometric calibration capabilities that are directly traceable to laboratory standards, i.e., no models of astronomical objects are required. Because these instruments do not employ telescopes nor have spectral resolving capability, their calibration is not entirely relevant to that of

\footnotetext{
60 http://www.cosmos.esa.int/web/planck/publications
} 
astronomical telescope-spectroscopic combinations, which are the focus of this paper. Nevertheless, we discuss total solar irradiance monitors in order to demonstrate the advantages of using laboratory standards in orbit.

The most accurate radiometric instruments used for this purpose are electrical substitution radiometers, such as the Active Cavity Irradiance Monitors (Willson 1999) on the Solar Maximum Mission (SMM, 1980 to 1989, Bohlin et al 1980 Chipman 1981), the Upper Atmosphere Research Satellite (UARS, 1991 to 2005, Reber et al 1993), and on the Active Cavity Radiometer Irradiance Monitor Satellite (ACRIMSAT, launched in 1999, Willson 2014), TIM on SORCE (launched 2003, Kopp et al 2005), the Differential Absolute Radiometers DIARAD and PMO6-V instruments that are part of the Variability of Solar Irradiance and Gravity Oscillations package (VIRGO, Fröhlich et al 1997) on SOHO (Fleck et al 1995) and of the SOLAR package on the International Space Station ISS. These ESRs operate at about $300 \mathrm{~K}$ and, in principle, have measurement uncertainties far below the percent level. For the primary calibration, radiometers operating at liquid helium temperatures can reach an SI uncertainty of approximately $5 \times 10^{-4}$ (500 ppm, Willson 2014). $\mathrm{TIM} / S O U R C E$ states an accuracy of $1 \times 10^{-4}$, with a precision of better than $1 \times 10^{-6}$. Details on the ground and flight calibrations of TIM are described by Kopp et al (2005). However, in order to make the results of various measurements agree with the space absolute radiometric reference scale (SARR, Crommelynck et al 1995), significant adjustments, depending not only on the specific instrument optics and geometry (such as, e.g., apertures, filters, and slits) but also on apparent instrument degradations and changes in observing parameters, have been required. Fröhlich (2013), Willson (2014) and others have repeatedly discussed such adjustments, and the various measurements of the total solar irradiance from spacecraft have been combined into composite time series by several authors.

Recently, Fröhlich has undertaken a thorough re-analysis of the test measurements performed in the laboratory on the flight spare instruments VIRGO 1 and VIRGO 4 in order to revise the characterisation of the PMO6V-A and PMO6V-B instruments which are still operating on SOHO. Similarly, he revised the characterisation of the DIARAD radiometer and thus obtained a new absolute value of the VIRGO TSI 61

The resulting composite reaching back to 1978 (Fröhlich and Lean 1998; Fröhlich 2015a b) is shown in Figure 13. It is noteworthy that the discrepancy between the scales of TIM/SORCE and VIRGO/SOHO is now such that the TSI averaged over the last minimum determined by VIRGO, $(1359.66 \pm 2.47) \mathrm{W} \mathrm{m}^{-2}$, deviates from the $1360.52 \mathrm{~W} \mathrm{~m}^{-2}$ measured by TIM by less than half its uncertainty. Preliminary calculations of a composite time series combining wavelet methods and Bayesian statistics (e.g., Dudok de Wit 2011, 2014, von der Linden et al 2014) have led to similar findings.

\section{Spectral irradiance measurements}

Spaceborne spectrometers with true, on-board, spectral irradiance calibration capabilities that permit efficiency changes to be tracked from the time of laboratory calibration through integration, launch, and years of use in orbit have been the Solar Ultraviolet Spectral Irradiance Monitors (SUSIM; VanHoosier et al 1988; Brueckner et al 1993). These have monitored with modest spectral resolving power and without a telescope the full-disk VUV and UV solar spectral irradiance in the wavelength band that drives the photochemistry of the Earth's ozone layer. The SUSIMs, which have observed the Sun several times from the Space Shuttle and the UARS, comprised several spectrometers and a number of $\mathrm{D}_{2}$-lamp transfer standards. The initial spectrometer calibrations were established in the laboratory by direct comparison with synchrotron radiation. During orbital

61 The corresponding references are accessible through the webpage http://www.pmodwrc.ch/pmod.php?topic=tsi/ composite/SolarConstant 


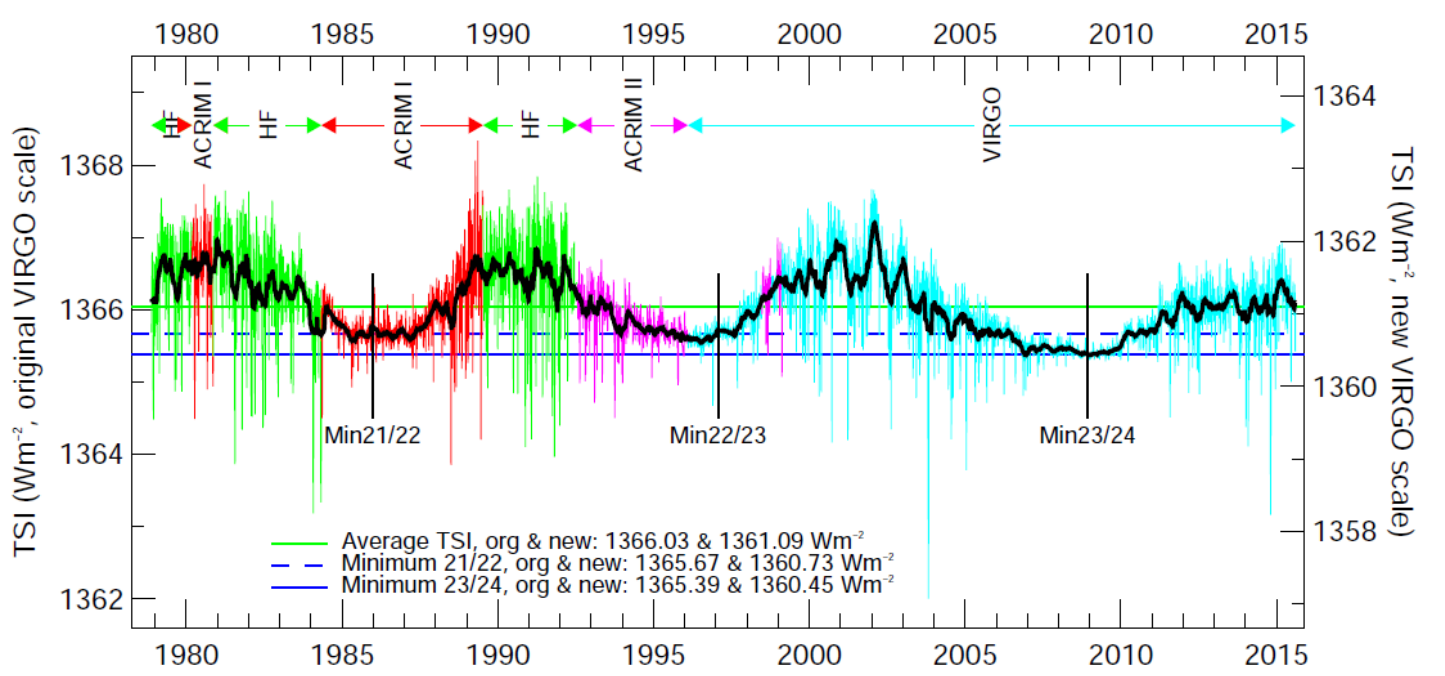

Fig. 13 The PMOD composite of total solar irradiance (TSI). Daily values (1978 to present) are plotted in different colours for the different experiments. The new and original VIRGO scales are shown on the right- and left-hand ordinates, respectively. Three numbers characterising the solar-activity cycles that have so far been covered by measurements from space are listed. (Courtesy of C. Fröhlich.)

operations, one of the spectrometers is not used to view the Sun, a procedure that could contribute to rapid degradation in performance, but only to monitor relative changes in the output of the $\mathrm{D}_{2}$ lamps, which have significantly different duty cycles, again to preserve the laboratory calibration on the lamp by less frequent operation.

The Solar Stellar Irradiance Comparison Experiment SOLSTICE (Rottman and Woods 1994) has also been part of the $U A R S$ instrument complement and monitored the full-disk solar spectral irradiance with approximately the same resolution and spectral range as SUSIM. SOLSTICE was thoroughly calibrated by use of synchrotron radiation before launch, but had no on-board calibration capability per se. Instead SOLSTICE tracked changes in its detection efficiency by comparing solar irradiances to those of hot stars. The set of these is large enough that changes in the instrument can be disentangled from unexpected stellar variability, which is thought to be negligible in the stars chosen, by comparing each star to the ensemble average. Difficulties with SOLSTICE measurements, as with similar other instruments, are: $(i)$, possible undetected change in the instrument efficiency between pre-launch calibration and in-orbit observations, and, (ii), the large dynamic range, about $10^{8}$, over which accurate radiometry is required. This range is accommodated by using different exposure times and a wide range of spectrometer entrance and exit apertures. However, the latter differences mean that the solar and stellar observations use different fractions of the spectrometer optics, which may be non-uniform in performance across their surface or which may change properties at different rates as a function of time. The UARS SUSIM and SOLSTICE instruments monitored the VUV solar irradiance regularly and simultaneously from 1992 to 2005. Woods et al (1996) compared the results and those of several other VUV solar irradiance monitors.

A next generation of solar spectral irradiance instruments is represented by the Solar EUV Experiment (SEE, Woods et al 1998) on the NASA Thermosphere Ionosphere Mesosphere Energetics and Dynamics $(T I M E D)$ spacecraft and the Spectral Irradiance Monitor (SIM, Rottman et al 1998, Lawrence et al 1998) on the SORCE mission. TIMED has been launched in December 2001 and provides VUV irradiance measurements using grating spectrographs and silicon photodiodes to cover the wavelength range $0.1 \mathrm{~nm}$ to $200 \mathrm{~nm}$. The EUV grating spectrograph (EGS) as well as the XUV (astro)photometer system (XPS) are advanced versions of earlier instruments used in rocket 
flights. Calibration as well as comparison with other spectral irradiance monitors is documented by Woods et al (2005) 62 Continued independent measurements with prototype instruments on rockets, scheduled approximately annually, provide data for in-flight calibration.

The Solar Radiation and Climate Experiment $(S O R C E)$ spacecraft was launched in January 2003, and, besides the Total Irradiance Monitor (TIM), carries the Spectral Irradiance Monitor (SIM), the Solar Stellar Irradiance Comparison Experiment (SOLSTICE), and the XUV Photometer System (XPS). Together they measure the solar spectral irradiance from $1 \mathrm{~nm}$ to 2 um, accounting for $95 \%$ of the spectral contribution to TSI, i.e., the so-called "solar constant". SIM/SORCE is particularly interesting because it incorporates a very sensitive ESR as a detector in its focal plane and a dual spectrometer arrangement that allows degradation in orbit to be tracked. The spectral range covers $310 \mathrm{~nm}$ to $2.4 \mathrm{\mu m}$, thus the visible and near infrared (Vis/NIR) range. An additional channel covers the $200 \mathrm{~nm}$ to $300 \mathrm{~nm}$ ultraviolet spectral region in order to overlap with the SOLSTICE instrument. SIM's accuracy amounts to $2 \%$ S3 SOLSTICE/SORCE provides precise daily measurements of solar spectral irradiance at ultraviolet wavelengths, covering $115 \mathrm{~nm}$ to $320 \mathrm{~nm}$ with a spectral resolution of $0.1 \mathrm{~nm}$, an absolute accuracy of better than $6 \%$, and a relative accuracy (precision) of $0.5 \%$ per year ${ }^{64}$ The SOLSTICE preflight calibrations included component-level calibrations in the LASP calibration laboratory and system-level calibrations primarily at the NIST Synchrotron Ultraviolet Radiation Facility SURF III, similar to those used for the SOLSTICE/ UARS calibration programme (Woods et al 1993). Unit-level calibrations included characterisation of individual optical and instrument elements, including gratings, detectors, and mirrors. The system-level calibrations included detailed wavelength and radiometric calibrations of the fully assembled instrument. Wavelength calibration used platinum and mercury lamps providing well-spaced emission lines. The in-flight calibration for the SOLSTICE instruments includes long-term multi-wavelength observations of stable early-type O-B stars and observations using redundant channels with strongly reduced duty cycles. The wavelength calibration for the in-flight data uses prominent solar emission features. The XPS (Woods and Rottman 2005) evolved from earlier versions flown on the Student Nitric Oxide Explorer (SNOE; Bailey et al 1999) and TIMED. It measures the solar soft X-ray (XUV) irradiance from $1 \mathrm{~nm}$ to $34 \mathrm{~nm}$ and the bright hydrogen emission at $121.6 \mathrm{~nm}$ (H I Lyman-alpha). The photodiodes were calibrated at NIST SURF-III with reference Si diodes (uncertainty of $2 \%$ ). In-flight calibration is achieved via redundant channels with lower (weekly) duty cycles and rocket flights, the latter resulting in an absolute irradiance uncertainty of about $12 \%$ to $24 \% 65$ Additionally, any other available solar XUV irradiance measurements, such as from SOHO and SEE/TIMED, are included in the XPS validation programme.

Yeo et al (2015) summarise current solar irradiance studies, measurements as well as models, and also come to the conclusion that despite a vast number of open questions remaining, the combined effort has led to major improvements in our understanding of the solar cycle and its variation.

\section{The SOLAR instruments on ISS}

The SOLAR package has been mounted in 2008 (after being carried into orbit aboard the shuttle mission $S T S$-122) as one of the external payloads of the Columbus laboratory on the International Space Station $(I S S)$. The total and spectral irradiance is recorded simultaneously in the $16 \mathrm{~nm}$ to $3.1 \mathrm{~m}$ range by three instruments.

62 http://lasp.colorado.edu/see/calibration.htm

63 http://lasp.colorado.edu/home/sorce/instruments/sim/

64 http://lasp.colorado.edu/home/sorce/instruments/solstice/

65 http://lasp.colorado.edu/home/sorce/instruments/xps/in-flight-calibration/ 
The Solar Auto-Calibrating EUV/UV Spectrophotometer (SolACES) operates three grazingincidence plane gratings to determine the absolute fluxes from $16 \mathrm{~nm}$ to $150 \mathrm{~nm}$ (Schmidtke et al 2014). Its fourth spectrometer stopped operating shortly after launch. The absolute calibration is achieved by primary standards, namely two rare-gas ionisation chambers (cf., Figure 14).

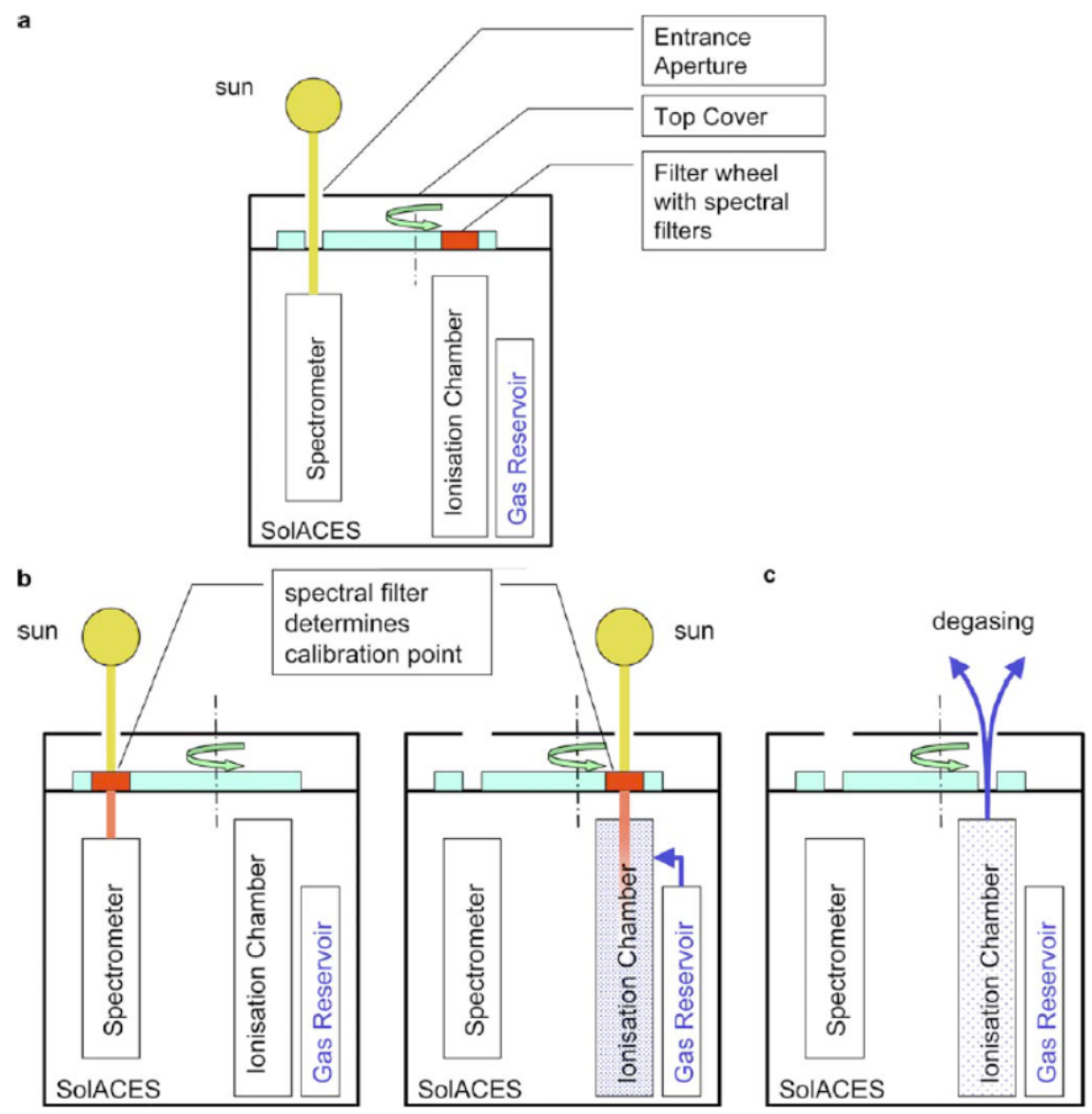

Fig. 14 (a): The scheme of the SolACES spectrometer - filter wheel - ionisation chamber arrangement. (b)-(c): The sequence of the calibration mode. (After Figure 6 in Schmidtke et al (2006), with permission).

During the mission, the instrument responses steadily declined due to ageing of the channel electron multiplier sensors and contamination. A special calibration sequence with various gases and filter settings is repeated regularly, at a higher frequency than originally foreseen, in order to monitor these changes. The overall uncertainty has been determined to be $10 \%$. The instrument description, its performance and results, as well as comparisons with SEE/TIMED and EVE/SDO (Woods et al 2012) in the EUV band between $16 \mathrm{~nm}$ to $25 \mathrm{~nm}$ are given by Schmidtke et al (2014). The agreement of all three data sets is well within $20 \%$ throughout the years 2008 to 2013 .

SOVIM (Mekaoui et al 2010) is combining two types of absolute radiometers and three-channel filter radiometers. The SOVIM package is composed of three PMO6 absolute radiometers, two solar photometers, one pointing sensor and one DIARAD radiometer.

SOLSPEC (Thuillier et al 2009) is composed of three double spectrometers using concave gratings, covering the wavelength range from $165 \mathrm{~nm}$ to $3.1 \mu \mathrm{m}$. Like SolACES it has been calibrated 
by use of primary standard sources of the PTB. Internal calibrations have been performed regularly, by measuring the spectrum of internal deuterium lamps (UV) or tungsten lamps (visible and IR) 66

All irradiance measurements in the UV range point out the importance of rigorous cleanliness and contamination control at instrument and spacecraft levels. Moreover, the use of novel materials, such as wide-bandgap semiconductors enhances the robustness of detectors against proton radiation (BenMoussa et al 2015).

\section{Solar irradiance spectra}

In addition to the measurements discussed above, which were mainly focussed on the UV and VUV wavelength ranges, calibrated solar spectra have been obtained from the ground over the past few decades (Arvesen et al 1969, Thekaekara 1974, Neckel and Labs 1984; Lockwood et al 1992, Burlov-Vasiljev et al 1995, Thuillier et al 2004a b). Most of this work is not directly relevant to the subject of this paper, but these measurements do contribute to the development of the solar reference spectrum and can be used to assess measurements from space in spectral regions where there are overlapping data.

During March/April 2008 (Carrington rotation 2068) and still within the extended last solar minimum (transition between solar cycles 23 and 24), solar irradiance reference spectra (SIRS) from $0.1 \mathrm{~nm}$ to $2.4 \mathrm{\mu m}$ have been generated using a combination of satellite and sounding rocket observations (Woods et al 2009). These reference spectra include daily satellite observations from SEE/TIMED and SORCE instruments. Data on the extreme ultraviolet range have also been improved with higher spectral resolution observations by use of the prototype SDO Extreme ultraviolet Variability Experiment (EVE) aboard a sounding rocket launched on 14 April 2008. Comparisons with the ATLAS-3 reference spectrum ${ }^{67}$ (Thuillier et al 2004a b) showed a significant overall improvement in uncertainties $(10 \%$ to $15 \%$ in the EUV and X-ray range, compared to the earlier $30 \%$ to $50 \% ; 2 \%$ to $5 \%$ compared to $3 \%$ to $10 \%$ in the FUV-MUV; around $1 \%$ to $3 \%$, both in the VIS and NIR) thanks to improved calibration and monitoring.

\section{Spectroradiometric telescopes on $\mathrm{SOHO}$}

The next serious efforts at accurate solar spectroradiometric measurements from space following the $O S O$ series and Skylab were associated with the $S O H O$ mission (Fleck et al 1995). The $S O H O$ project required extreme cleanliness in construction, integration, and launch operations so that changes in performance in orbit would be minimised. The spacecraft builders worked with a cleanliness requirement of a few hundred nanograms of condensable and particulate contamination per square centimetre, while the instrument teams aimed for even less. The minimal deterioration in performance of these instruments over the entire course of the mission is attributable, probably to a large extent, to the cleanliness achieved (Thomas 2002).

The spectroradiometric efficiency of the Solar Ultraviolet Measurements of Emitted Radiation instrument on SOHO (SUMER, Wilhelm et al 1995) was determined prior to launch by employing a source standard that had been calibrated by comparison to synchrotron radiation (Hollandt et al 1996). The source standard consisted of a hollow cathode which emitted a line spectrum, and a spherical normal-incidence collimating mirror. The collimated beam had a $10 \mathrm{~mm}$ diameter and a divergence of $\pm 1^{\prime}$. In the laboratory calibration of SUMER, the image of the flux-limiting aperture of the hollow cathode underfilled the aperture as seen from the focal plane of the telescope, which is, at the same time, the entrance slit plane of the spectrometer. An unobstructed observation of

66 http://solspec.projet.latmos.ipsl.fr/SOLSPEC_GB/Home.html

67 taken during the Atmospheric Laboratory for Applications and Science (ATLAS) Space Shuttle missions ATLAS 1, March 1992, and ATLAS 3, November 1994 


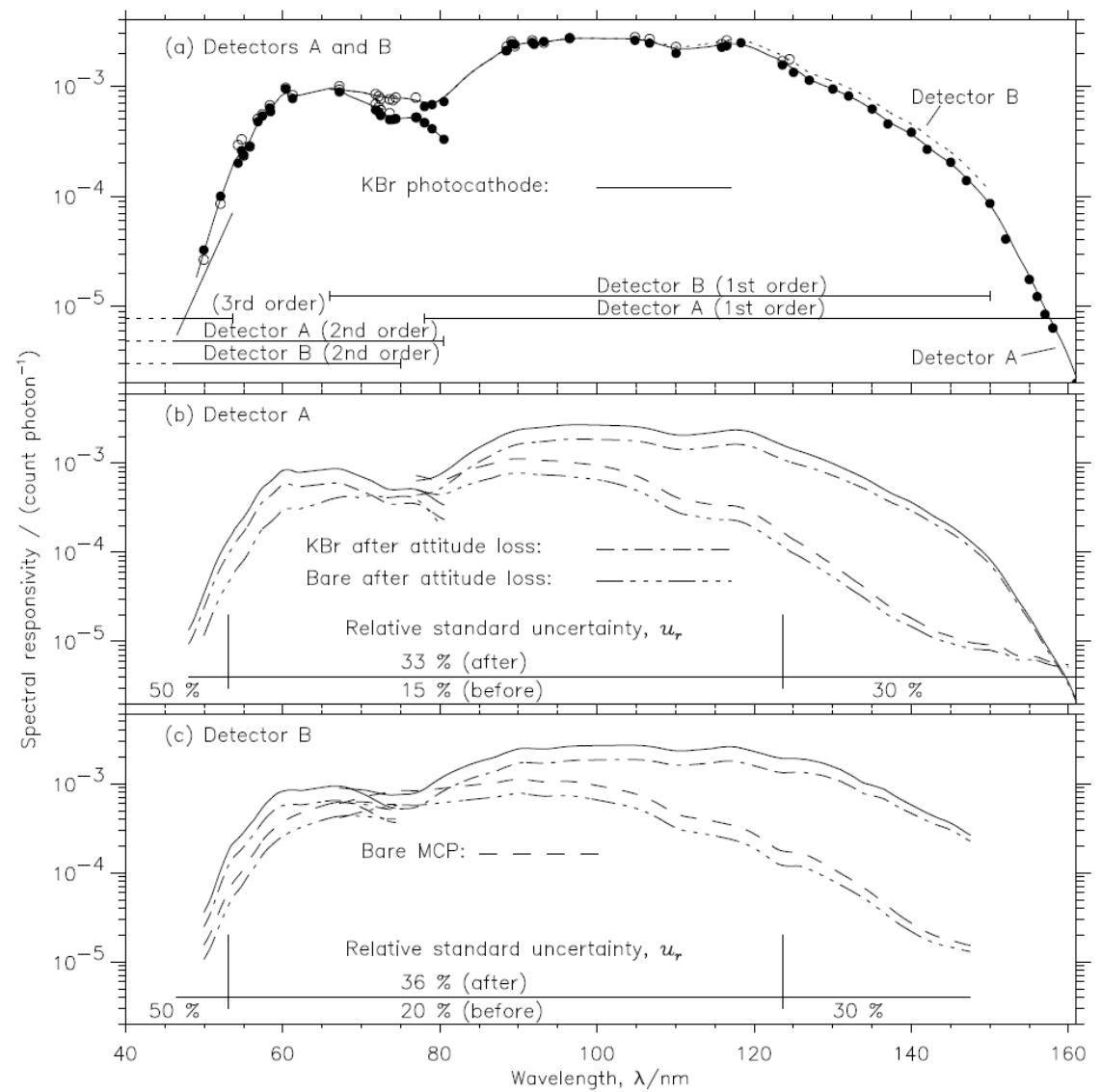

Fig. 15 Spectral responsivities of the SUMER spectrometer and the corresponding relative uncertainties. (a) Firstorder, second order, and third-order responsivities for both detectors (interpolated between calibration points); (b) Independent detector A assessment; and (c) results for detector B. The relative uncertainties in the central wavelength range (indicated by vertical bars at $53 \mathrm{~nm}$ and $124 \mathrm{~nm}$ ) are derived from the laboratory calibration and are smaller than those of the in-flight calibration extensions obtained from calculated line-ratios (i.e., branching fractions) and stellar observations. (After Figure 1 in Wilhelm et al (2002), with permission).

the entire source radiation was thus achieved and spectroradiometric responsivities that included the reflectivities of all optical surfaces, the grating efficiencies, and the detector performance could be established by an appropriate scan of the entrance aperture ${ }^{68}$ The pre-launch calibrations have subsequently been further refined under operational conditions (Wilhelm et al 1997; Schühle et al 2000). SUMER has been measuring with two detectors, A and B, in almost overlapping wavelength bands. The central part of both detectors' photocathode microchannel plate (MCP) has a coating of potassium bromide $(\mathrm{KBr})$ and the other parts use the bare plate. The spectral responsivity characterisation of the instrument is shown in Figure 15, and the VUV radiance spectrum of a quiet-Sun region with prominent emission lines and continua in the wavelength range from $80 \mathrm{~nm}$ to $150 \mathrm{~nm}$ is presented in Figure 16.

The spectroradiometric efficiency of the Ultraviolet Coronagraph Spectrometer on SOHO(UVCS Kohl et al 1995) was determined before launch at selected wavelengths by use of transfer-standard photodiodes (Gardner et al 1996). The results, which had an estimated uncertainty of $20 \%$, have

68 Note however that apertures and stops in the instrument itself were not taken into account in this way. 


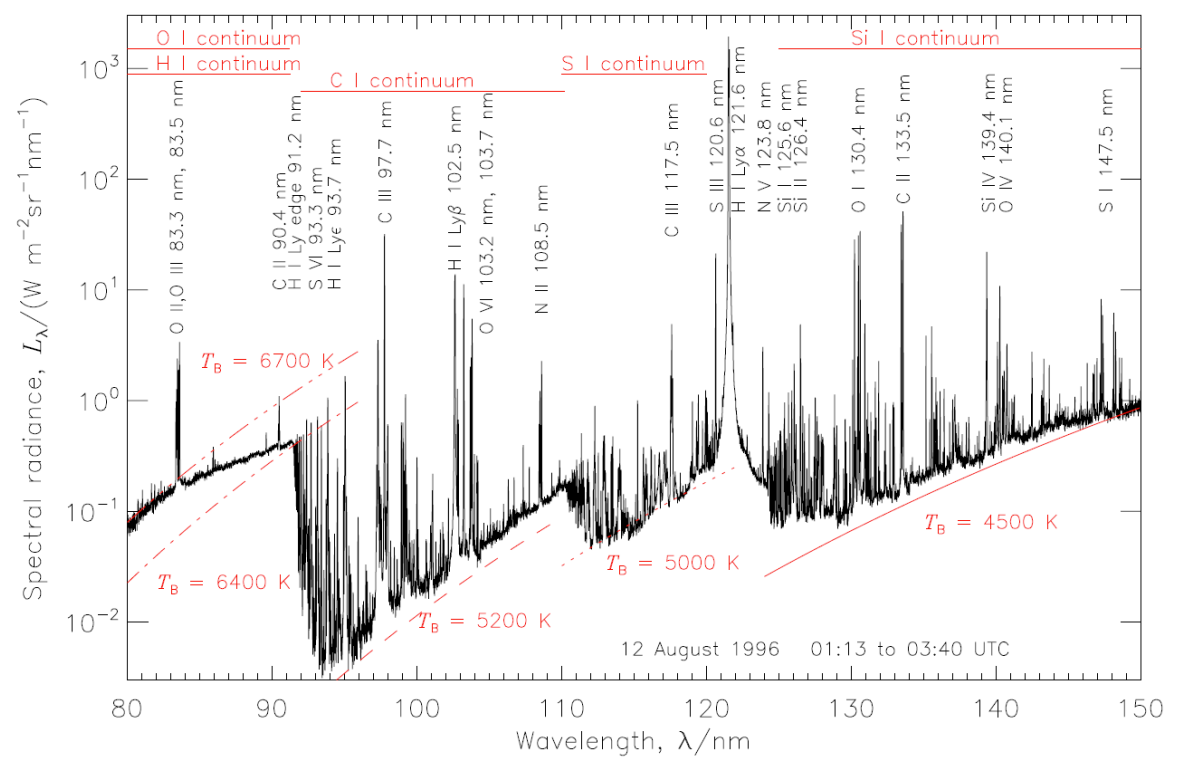

Fig. 16 Spectral radiance of the quiet Sun in the VUV range from a region near the centre of the disk, measured with the SUMER detector on SOHO. The spectral radiances of some continua expected for certain brightness temperatures, $T_{\mathrm{B}}$, are also shown. The relative uncertainty below $124 \mathrm{~nm}$ is $15 \%$ and above this limit $30 \%$. (After Figure 4 in Wilhelm (2010), with permission).

been confirmed for the instrument parameters used in the laboratory by underflights with the SPARTAN 201 Shuttle-borne spectrometer (Frazin et al 2002). Annual observations of stars that pass through the UVCS field of view have shown that there has been no detectable (at the $10 \%$ to $15 \%$ level) change of responsivity over the course of the mission. Because of limited resources and instrument limitations, it was not possible before launch to calibrate UVCS over the full range of instrument parameters used in science observations. In-orbit studies and laboratory tests using replicas of the flight gratings have shown that, because the reflectivity of the grating is not uniform across its surface, the UVCS O VI channel effective area is not a linear function of the width of the aperture 69 (Gardner et al 2000).

Although UVCS did not rely on extensive modelling to determine instrument performance parameters, this experience is instructive to those who attempt to model the performance of complex instruments from first principles: It is often difficult to predict the behaviour of state-of-the-art optical components, including detectors, and coatings (and the inevitable contaminants) over the complete range of physical dimensions, optical angles, wavelengths, etc., expected in use. The experience of the $O S O-6$ and CDS (discussed below) laboratory calibration teams, who had to deal with non-uniform beams reinforces this point. Thus performance models should be verified with benchmark measurements over as much of the parameter space as possible.

The spectroradiometric efficiency of CDS, the Coronal Diagnostic Spectrometer (Harrison et al 1995), was measured prior to launch in a manner similar to that used for SUMER: the source standard used was also calibrated by comparing it to synchrotron radiation (Harrison et al 1996), but in this instance, a Wolter type-II telescope served as the collimator and the collimated beam was limited to $5 \mathrm{~mm}$ in diameter when it left the Wolter telescope. The nominal divergence was $\pm 30^{\prime \prime}$. Inside its vacuum tank, the CDS instrument could be moved perpendicular to the beam (while the

69 UVCS is a coronagraph with external and internal occulters. Adjustment of the latter, which is done to reduce the level of scattered light, changes the aperture. 
optical axis was maintained), so that the instrument apertures that illuminated the grazing- and normal-incidence gratings could be mapped. The laboratory calibration of CDS (Lang et al 2000) turned out to be a much more complex undertaking than that of SUMER. Several reasons contributed to this, not the least being the fact that the collimated beam showed structure in its cross section and exhibited an angular divergence exceeding its nominal value by nearly a factor of four. Rocket underflights (a NASA/LASP rocket carrying a photoelectric EUV Grating Spectrograph, EGS, and SERTS-97, the Solar EUV Rocket Telescope and Spectrograph) and line-ratio techniques were used to reassess the initial calibration at early stages of the mission (Brekke et al 2000; Del Zanna et al 2001). Later on, an update was performed using data from intercalibration campaigns and from two Extreme-Ultraviolet Normal Incidence Spectrograph (EUNIS) rocket flights 2006 and 2007. As described by Wang et al (2011), the EUNIS spectrographs had been calibrated at RAL against the same transfer standard (cross-calibrated against the BESSY II storage ring as primary source) that had been used for the calibration of CDS and the EIS on Hinode (see below).

The Extreme ultraviolet Imaging Telescope EIT has been designed to provide full-disk solar images of the lower corona in four EUV passbands (Delaboudinière et al 1995). It consists of a normal-incidence Ritchey-Chrétien telescope with multilayer coating and a back-side illuminated thinned CCD sensor at its focus. The pre-flight spectral response had been calibrated at the Institut d'Astrophysique Spatiale (IAS) in Orsay using synchrotron light from the SuperACO positron storage ring (Defise et al 1995). Several parameters and calibration components could only be determined in-flight using special operation protocols and sequences, such as determining the point spread function (PSF) via flare observations and a Mercury transit (Clette et al 2002). Furthermore, the EIT has been suffering from time-varying response degradation, partially induced by contaminants on the CCD and on optical surfaces, and also by radiation damage to the CCD. Some non-polymerized contaminants, such as water, could successively be eliminated via heating of the components; however, as the water re-condensed at the instrument's housing wall, this led to oscillatory behaviour, especially during the first years of the mission. The flat-field of the CCD and its variation are modelled comparing and correlating it to flat-fields in the visible range measured by use of calibration lamps (see, e.g., BenMoussa et al 2013, and references therein).

The Solar EUV Monitor (SEM, Hovestadt et al 1995) comprises a free-standing, 5000 lines $/ \mathrm{mm}$ transmission grating, aluminum-coated silicon photodiodes, and aluminum filters that define the bandpass. It has been designed to measure the He II $30.4 \mathrm{~nm}$ output from the Sun as well as the integrated flux between $17 \mathrm{~nm}$ and $70 \mathrm{~nm}$. The SEM showed steady degradation of the first-order signal over the first seven years of operation. After that the responsivity has remained almost constant. This result followed from a series of dedicated calibration-rocket underflights (McMullin et al 2002; BenMoussa et al 2013).

The LASCO experiment (Brueckner et al 1995) has been designed as a set of three independent coronagraph telescopes to record white-light images of the solar corona from $1.1 \mathrm{R}_{\odot}$ through $30 \mathrm{R}_{\odot}$. After SOHO's temporary loss of attitude in 1998, the C1 instrument observing the innermost range stopped functioning. The $\mathrm{C} 2$ instrument covers the geometric range from (2.5 to 6.5) $\mathrm{R}_{\odot}$, and the C3 field of view extends from (3.8 to 30) $\mathrm{R}_{\odot}$. Pre-flight calibration of the LASCO instruments has been performed at the Naval Research Laboratory (NRL). The radiometric in-flight calibration is based on on-board calibration lamps as well as on a set of stars that have to be observed against the background of the solar corona (Llebaria et al 2004, Thernisien et al 2006; Morrill et al 2006).

Intercalibration data sets, taken on a regular basis for SUMER and CDS, in selected areas and conditions turned out to be extremely valuable, in particular to re-evaluate calibrations after SOHO's loss of attitude and subsequent recovery. Various detector parts of CDS (normal incidence, NIS, and grazing incidence, GIS) and SUMER (detctors A and B) had been compared in dedicated observing sequences of overlapping solar areas and in several EUV lines. Differences of $2 \%$ to $50 \%$ were found, and could be used to re-evaluate the calibrations (Pauluhn et al 1999, 2001, 2003). In- 
flight calibration studies were also made employing atomic data bases and the line-ratio technique (Del Zanna et al 2001, 2010), and, subsequently, rocket underflights were used to update the CDS calibration (Wang et al 2011). For various instruments, such as CDS and EIT, the spectrally summed radiances could be compared to irradiance monitors, such as SEM (Thompson et al 2002). More details are given in the ISSI report dedicated to SOHO's radiometric calibration (Pauluhn et al 2002) and in the article by BenMoussa et al (2013) which summarises the experience with stability of solar space radiometric instrumentation in great detail.

\section{Spectroradiometric telescopes on TRACE}

The Transition Region and Coronal Explorer ( $T R A C E$, Handy et al 1999) was a NASA SMEX mission operating from 1998 to 2010 that imaged the solar photosphere, transition region, and corona with angular resolution of $1^{\prime \prime}$. To provide continuous coverage of solar phenomena, TRACE was located in a sun-synchronous polar orbit. The instrument featured a $30 \mathrm{~cm}$ Cassegrain telescope, multilayer optics, and a lumogen-coated CCD detector to record three EUV and several UV wavelengths. The pre-flight calibration had been performed partially in the laboratory. Measurements of the quantum efficiencies of the CCDs together with the lumogen coating, for example, were made at the Lockheed Martin ATC70 facility. The subsequent on-orbit characterisation of the filters and the consecutive updates and monitoring of the flat fields has been described by Handy et al (1999). Reference spectra from SOLSTICE and the shuttle- or rocket-borne High Resolution Telescope and Spectrograph (HRTS, Handy et al 1998), as well as comparisons with SUMER/SOHO observations (Kim et al 2006), were used to "clean" the spectrum, i.e., correct for

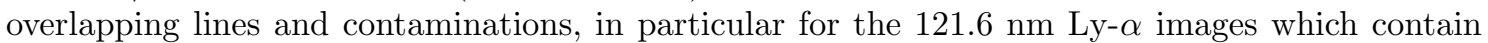
a contribution from the UV continuum at wavelengths beyond $150 \mathrm{~nm}$ and for the C IV doublet which needs to be deconvolved from several contributions.

\section{Telescopes on STEREO}

The STEREO mission, launched in 2006 (Kaiser et al 2008b), consists of two nearly identical observatories, one ahead of the Earth on its orbit (STEREO-A, Ahead), the other trailing behind (STEREO-B, Behind). Each spacecraft carries four instrument packages for measuring plasma properties of solar energetic particles, the magnetic field, and radio disturbances, as well as a set of telescopes to investigate the characteristics of coronal mass ejections (CME). Since February 2011, the two spacecraft have been providing unprecedented views of the far side of the Sun, and, together with data from the Solar Dynamics Observatory $(S D O)$, collected $360^{\circ}$ maps of the Sun. From late 2014 to early 2016, the STEREO spacecraft will be in a position in space where the Sun will affect full data downloads and the communication will be restricted. At least one spacecraft, however, will always be collecting data.

STEREO's Sun Earth Connection Coronal and Heliospheric Investigation (SECCHI) package comprises five telescopes for imaging the solar corona from the solar disk to beyond 1 au. These telescopes are: an extreme ultraviolet imager (EUVI: (1 to 1.7) $\mathrm{R}_{\odot}$ ), two Lyot coronagraphs (COR1: (1.5 to 4$) \mathrm{R}_{\odot}$ and COR2: (2.5 to 15$) \mathrm{R}_{\odot}$ ) and two heliospheric imagers (HI-1: (15 to 84) $\mathrm{R}_{\odot}$ and HI-2: (66 to 318$) \mathrm{R}_{\odot}$ ). All instruments use $2048 \times 2048$ pixel CCD arrays in a backside-illuminated mode. Their backside surface has been specially processed for EUV sensitivity, while the other has an anti-reflection coating applied. The EUVI mirrors were calibrated at the synchrotron of the IAS in Orsay and its CCDs at the Brookhaven synchrotron and the LMSAL XUV calibration facility (Howard et al 2008) 71

\footnotetext{
70 The Lockheed Martin Advanced Technology Center recently changed its name to Space Technology Advanced Research and Development Laboratories, or STAR Labs.

71 see also http://secchi.Imsal.com/EUVI/
} 
The Heliospheric Imager (HI) is a wide-angle, visible-light, imaging system for the detection of CME events in interplanetary space and, in particular, of events directed towards the Earth. Large parts of its radiometric calibration and the monitoring thereof have been made by use of stars of known minor variability as reference ${ }^{72}$ (Brown et al 2009, Bewsher et al 2010, 2012). No significant deterioration has been found (BenMoussa et al 2013$)$.

The paired COR1 telescopes, the innermost coronagraphs of the SECCHI instrument suite, observe the white-light K-corona from (1.4 to 4$) \mathrm{R}_{\odot}$ in a waveband $22.5 \mathrm{~nm}$ wide, centered on the $\mathrm{H} \alpha$ line at $656 \mathrm{~nm}$. Observations of the planet Jupiter are used to establish absolute radiometric calibrations for each telescope. Thompson and Reginald (2008) have described the radiometric and pointing calibration 73

\section{Spectroradiometric telescopes on Hinode}

The Japanese Hinode mission, launched in 2006, supports three solar telescopes, co-funded by JAXA-ISAS, NASA, ESA, and the United Kingdom Space Agency (UKSA). They cover the optical range (Solar Optical Telescope, SOT), the EUV range (EUV Imaging Spectrometer, EIS) and the X-ray range (X-Ray Telescope, XRT). The EIS pre-launch radiometric calibration has been performed at RAL in the same way as that of CDS (Seely et al 2004, Lang et al 2006, Culhane et al 2007; Brown et al 2008). The calibration is regularly monitored in orbit; this includes flat-fields from LED exposures, dark images, quartz-crystal microbalance (QCM) measurements, as well as dedicated observation campaigns. The latter have included EUNIS rocket flights that were also used for the CDS/SOHO calibration (Wang et al|2011).

\section{Spectroradiometric telescopes on SDO}

The Solar Dynamics Observatory $(S D O)$, which has been extensively described by Pesnell et al (2012) in a special edition of Solar Physics, has been launched in February 2010. SDO carries three scientific experiments; the Atmospheric Imaging Assembly AIA (Lemen et al 2012), the EUV Variability Experiment EVE (Woods et al 2012), and the Helioseismic and Magnetic Imager HMI (Schou et al 2012).

EVE measures the solar spectral irradiance from from $0.1 \mathrm{~nm}$ to $105 \mathrm{~nm}$ at $0.1 \mathrm{~nm}$ resolution. EVE's high-resolution irradiance instruments are the Multiple EUV Grating Spectrographs: MEGS-A, a grazing-incidence spectrograph that measures the solar EUV irradiance in the $6 \mathrm{~nm}$ to $37 \mathrm{~nm}$ range ${ }^{74}$ and MEGS-B, a normal-incidence, dual-pass spectrograph that measures the solar EUV irradiance in the $35 \mathrm{~nm}$ to $105 \mathrm{~nm}$ range. To provide their in-flight calibration, an EUV spectro-photometer (ESP) measures the solar EUV irradiance in broad bands between $0.1 \mathrm{~nm}$ and $39 \mathrm{~nm}$, and a photometer measures the Sun's bright Ly- $\alpha$ emission at $121.6 \mathrm{~nm}$. The ESP instrument on SDO was calibrated at NIST's SURF III before and after integration with the EVE package, and the ground calibration results are available via the Laboratory for Astronomy and Space Physics' (LASP) webpage ${ }^{75}$ A nominally identical instrument is periodically flown on a sounding rocket and makes observations of the Sun simultaneously with its SDO analogue. The rocket instrument is calibrated at SURF III's EUV detector radiometry beamline before and after each rocket flight, and the NIST calibration is transferred to the SDO instrument. In this way, EVE maintains an absolute calibration accuracy of better than $25 \%$ (Woods et al 2012, Hock et al 2012; Didkovsky et al 2012).

72 http://www.stereo.rl.ac.uk/Documents/InstrumentPapers.html

73 http://cor1.gsfc.nasa.gov/publications/

74 The MEGS-A had to be turned off due to a failure of its CCD electronics in May 2014.

75 http://lasp.colorado.edu/home/eve/data/ground-calibration-results/ 
The AIA instrument is an array of four normal-incidence reflecting telescopes that image the Sun in seven EUV, two VUV, and one visible wavelength channels. The initial pre-flight radiometric calibration of AIA was based on measurements of the response of all the telescope components (mainly at LMSAL and the Advanced Light Source (ALS) synchrotron at Lawrence Berkeley National Laboratory), which were then combined analytically into a model of the overall system performance. The dominating terms are the uncertainty in the contamination thickness and the CCD quantum efficiency determination. The accuracy of the preflight calibration has been estimated to be of the order of $25 \%$ (Boerner et al 2012). For tracking degradation (e.g., by contamination) and to refine and maintain the calibration, cross-calibration with EVE is routinely performed. Comparisons and cross-calibrations have also been performed between AIA and EVE/SDO, EIS/Hinode and SOLSTICE/SORCE (Boerner et al 2014). They found that most channels are in agreement within the uncertainties. The $30.4 \mathrm{~nm}$ channel showed a degradation by a factor of three from May 2010 through September 2011, very likely due to contamination effects. Some other inconsistencies could be ascribed to errors in the wavelength response function either of AIA or of EVE. Additionally, it was found that the version of the CHIANTI spectral lines data base then in use was missing some lines and underrepresenting emission in others.

\section{Instruments on IRIS}

The Interface Region Imaging Spectrograph (IRIS, De Pontieu et al 2014) SMEX spacecraft has been launched in June 2013 and provides simultaneous spectra and images of the photosphere, chromosphere, transition region, and corona with $0.33^{\prime \prime}$ to $0.4^{\prime \prime}$ spatial resolution, two-second temporal resolution, and $1 \mathrm{~km} / \mathrm{s}$ velocity resolution over a field-of-view of up to $175^{\prime \prime} \times 175^{\prime \prime}$. The IRIS instrument is a multi-channel imaging spectrograph with a $20 \mathrm{~cm}$ UV telescope, feeding $(i)$ two far-UV channels of $133.2 \mathrm{~nm}$ to $135.8 \mathrm{~nm}$ and $138.9 \mathrm{~nm}$ to $140.7 \mathrm{~nm}$ with $2.6 \mathrm{pm}$ resolution and effective areas of $1.6 \mathrm{~cm}^{2}$ and $2.8 \mathrm{~cm}^{2}$, respectively, and (ii) a near-UV channel of $278.3 \mathrm{~nm}$ to $283.5 \mathrm{~nm}$ with $5.3 \mathrm{pm}$ resolution and an effective area of $0.2 \mathrm{~cm}^{2}$. A large fraction of the radiometric calibration as well as tracking longterm contamination and degradation is achieved via observation of a selected set of UV-bright stars. As described in Section 3 the spectral radiances of such stars are given with an uncertainty of $10 \%$ to $15 \%$. IUE spectra of these stars can be viewed on the website of the MAST ${ }^{76}$ Stellar comparisons and quiet-Sun studies are planned for longterm monitoring of the calibration. The relevant information is also found on the corresponding webpages 77

\section{Solar X-ray spectroscopy}

Yohkoh (before launch called $S O L A R$ - $A$, Ogawara et al 1991) was a solar observatory operated from 1991 to 2001 by the Institute of Space and Astronautical Science (ISAS, Japan) in collaboration with the space agencies of the United States and the United Kingdom. It carried four instruments: a Soft X-ray Telescope (SXT), a Hard X-ray Telescope (HXT), a Bragg Crystal Spectrometer (BCS), and a Wide Band Spectrometer (WBS). SXT used already a CCD for readout. Part of the calibration had been performed at Rutherford Appleton Laboratory (Lang et al 1993).

The Reuven Ramaty High-Energy Solar Spectroscopic Imager (RHESSI, Lin et al 2002) is a NASA SMEX mission designed to investigate particle acceleration and energy release in solar flares, through imaging and spectroscopy of hard X-ray and gamma-ray continua emitted by energetic electrons, and of gamma-ray lines produced by energetic ions. The single instrument consists of an imager, made up of nine bi-grid rotating modulation collimators (RMC), in front of a spectrometer

\footnotetext{
$7 6 \longdiv { \text { http://archive.stsci.edu/ } }$

77 http://iris.Imsal.com/documents.html
} 
with nine cryogenically-cooled germanium detectors, one behind each RMC. RHESSI observes solar photons over three orders of magnitude in energy (3 keV to $17 \mathrm{MeV}$ ) with high energy resolution. In the range from $3 \mathrm{keV}$ to $100 \mathrm{keV}$, the resolution is about $1 \mathrm{keV}$ FWHM; in the range over $1 \mathrm{MeV}$, it is about $3 \mathrm{keV} \mathrm{FWHM} \mathrm{(Smith} \mathrm{et} \mathrm{al} \mathrm{2002).} \mathrm{The} \mathrm{gain} \mathrm{calibration} \mathrm{of} \mathrm{the} \mathrm{detector}$ segments is re-calculated on a regular basis using background data collected over a full orbit. The channel-to-energy conversion is represented as a purely linear fit over each energy scale. The effective area and response of the spectrometer has been simulated and reproduced by GEANT3, a high-energy photon and particle transport code that is used to generate the response matrix of the instrument. Before flight, the GEANT3 performance was calibrated by taking spectrometer data in the laboratory using radioisotopes with lines from $3.7 \mathrm{keV}$ to $6.1 \mathrm{MeV}$, placed in many positions above and around the spectrometer. The laboratory data were compared with GEANT3 simulations of the same configurations, and the detectors internal segmentation boundaries in GEANT3 were adjusted until the GEANT3 results matched the data. For monitoring the detector response over time and noting radiation damage, RHESSI carries a weak onboard radioactive source (5 nanocuries of ${ }^{137} \mathrm{Cs}$ ) emitting at $662 \mathrm{keV}$, far from any line expected to occur in flares or in the variable background. Background is usually high in the instrument, due to limited shielding (a consequence of weight constraints) and the chosen orbit, crossing the South Atlantic Anomaly (SAA). At most energies the continuum is dominant, which is typical for an unshielded instrument.

Hinode's X-Ray Telescope (XRT) ${ }^{78}$ was designed and developed by the Japan-US collaboration between the Smithsonian Astrophysical Observatory (SAO), the NASA Marshall Space Flight Center MSFC, JAXA, and the National Astronomical Observatory of Japan. The XRT CCD camera was tested and calibrated in X-rays at the Advanced Technology Center of the NAOJ with JAXA. The telescope calibration has been, among other locations, performed at the X-Ray Calibration Facility (XRCF) of the MSFC, and filters for the XRT were tested at the X-Ray Astronomy Calibration and Testing (XACT) facility of the Osservatorio Astronomico di Palermo (Golub et al 2007). The detector calibration is described by Kano et al (2008).

\section{Conclusions and Outlook}

Ultimately, it must be realised that an unambiguous deconvolution of observed signals into valid, scientifically interesting, time-dependent data is possible only if an instrument's spectral detection effciency can be completely characterised at all times. This requires either direct on-board calibration systems or highly-sophisticated component and system performance models supplemented with regular benchmark observations using equipment that is traceable to laboratory primary standards.

For the sake of completeness, we should mention here that this applies not only to the radiometric calibration of a space telescope. An overall calibration of a spectrometric space telescope requires that a considerable number of additional quantities be determined. These include: the spacecraft reference frame, the telescope's pointing accuracy and stability and its vignetting function, the point-spread function (PSF, both on- and off-axis), flat-field maps, stray light and the occurrence of ghost images and other backgrounds, the plate-scale, radiometric non-linearities owing to pulse pile-up or charge-transfer inefficiency, as well as the timing of the observations. As some of these quantities are prone to change during orbital operations - sometimes also as a consequence of particular events like spacecraft eclipses or large solar flares - in-orbit monitoring at appropriate intervals and following such events is necessary. Curiously, today still, more effort is often spent on such monitoring than on a proper radiometric laboratory calibration. These in-orbit comparisons are extremely vital and by no means rendered unnecessary by a solid pre-launch ca-

\footnotetext{
$7 8 \longdiv { \text { http://hinode.nao.ac.jp/ http://xrt.cfa.harvard.edu/ } }$
} 
libration, yet a thorough characterisation and understanding of the instrument before launch is a necessity for obtaining optimal measurements. There are, fortunately, indications that the "launchnow-calibrate-later" attitude is changing, not least fostered by the demanding requirements of some current and many future missions.

The terrestrial atmosphere has always hampered ground-based observations, be it by its turbulence or its tendency to absorb and even hide the radiation of cosmic objects arriving at Earth. Balloon and space astronomy have successively given access to more and more regions of the electromagnetic spectrum and with an image quality that lies beyond what can be attained on the ground. Past and current astrometric space missions have provided accurate distance measurements for a large number of stars; modern ground-based interferometry, moreover, has provided stellar radii. In principle, thus, it should now be possible to determine comprehensive properties of a large number of stars and other cosmic objects with high accuracy.

The radiometric calibration of space telescopes, a prerequisite to this goal has, however, turned out to be far from trivial. The environment to which an instrument after its laboratory calibration is exposed during storage and integration into the spacecraft, during transportation, preparations for launch as well as, finally, upon exposure to the environment of space, has a varying influence on its responsivity - and both improvement and degradation are possible (cf., Fig. 9). To overcome this problem, an extensive network of standard stars has been established. This approach becomes problematic, however, if one wants to achieve accuracies below a few per cent. When testing stellar models with this kind of calibration, there is a danger of circular conclusions.

For high-energy astronomy, standard candles, which here take over the role of stable standard stars, are rare, and changes of the radiometric calibration during operation in space occur as well. Under these circumstances an "International Astronomical Consortium for High Energy Calibration" whose members perceive radiometric calibration as a dynamic process has led progress. IACHEC encourages consideration of the derived constraints in the data exploitation and in scientific publications. They now see "success after years of diverging results on calibrations, and lack of communication among the teams". They emphasise on their web site that no important change in effective area of an instrument can take place devoid of other instruments' results.

In the sub-sections above, we have separately discussed spectroradiometry and associated accuracies for different wavelength ranges. We need to emphasise, however, that the radiometric calibration of telescopes should cover the entire spectral range accessible in space with similar accuracies. Investigations in multi-wavelength astronomy may address different phenomena, but such phenomena tend to be linked - be it by gravitation or by radiative, magnetic and mechanical energy or by particle transport. The solar corona, for example, is best observed through VUV and soft X-ray radiation, while the photosphere's characteristics are accessible through observations in the visible and infrared. A meaningful study of the links between phenomena observed in these diverse wavelength regions does require or, at least, would benefit from similar radiometric accuracies.

A move away from involving models of celestial standards in calibration to physics-based radiometric calibrations of space telescopes now seems to be underway, at least in the visible and near-infrared. Both the use of calibration rockets and calibrated ground-based telescopes supplemented by measurements of the prevailing properties of the terrestrial atmosphere are foreseen. This promises a future with one-per-cent and even sub-per-cent accuracies, traceable to Earthbased laboratory standards.

The recent turnaround in attitude towards the importance of an accurate spectroradiometric calibration, which has to some extent also been motivated by the discovery of dark energy, recognises the influence of calibration on the progress of science in hindsight. Looking forward, the asset of an advanced calibration is now recognised as well, specifically for $H S T$ it is pointed out that "Hubble's power to revolutionise has never been greater than now. The instruments are calibrated 
better than ever, and we are using them in ways we had not anticipated at the time of the last servicing mission" (Sembach 2015).

Nearly a decade ago|Mironov et al (2007) incited the participants at a conference on photometry to "turn from practical astrophysics to astronomical metrology." If this were to take hold for general spectroradiometry, we may look forward to sound progress in all fields of astronomy.

Acknowledgements The authors sincerely thank K. Bennett, C. T. Bingham, R. C. Bohlin, D. J. Coletti, T. Dudok de Wit, C. Fröhlich, L. D. Gardner, A. Gottwald, U. Grothkopf, J. B. Holberg, J. Hollandt, C. V. H. HuberOtt, F. Jansen, C. Jones, E. M. Kellogg, R. M. Klein, J. L. Kohl, J. W. Kruk, M. Kühne, X. Liu, R. Paladini, W. H. Parkinson, G. Schmidtke, J. G. Timothy, G. Ulm, K. Wilhelm, C. Winkler and B. J. Wargelin for illuminating discussions, reference material, both published and unpublished, and other assistance. PLS thanks the Space Science Department of ESA for travel support. He was also supported in part by NASA Grants NAGW-1596, and SOHO, and SPARTAN grants.

\section{List of acronyms}

\section{Missions}

AKARI Japanese space mission for infrared astronomy, formerly Astro-F

ANS Astronomical Netherlands Satellite

$A X A F$ Advanced X-ray Astrophysics Facility, now Chandra

BeppoSAX Italian-Dutch satellite for X-ray astronomy

Chandra X-ray observatory, formerly $A X A F$

COBE Cosmic Background Explorer

CXO Chandra X-ray observatory

Euclid Future ESA mission to map the geometry of the dark Universe

EUVE Extreme Ultraviolet Explorer

EXOSAT European X-ray Observatory Satellite

FUSE Far-Ultraviolet Spectroscopic Explorer

Gaia ESA astrometry mission

GALEX Galaxy Evolution Explorer

Herschel ESA infrared and sub-millimetre telescope mission

Hinode Solar observatory, formerly Solar-B

HST Hubble Space Telescope

IRAS Infrared Astronomy Satellite

IRIS Interface Region Imaging Spectrograph

IRTS Infrared Telescope in Space

ISO Infrared Space Observatory

ISS International Space Station

IUE International Ultraviolet Explorer

JWST James Webb Space Telescope

$M S X \quad$ Midcourse Space Experiment

NuSTAR Nuclear Spectroscopic Telescope Array

$O A O$ Orbiting Astronomical Observatory

ORFEUS Orbiting Retrievable Far and Extreme Ultraviolet Spectrometer

OSO Orbiting Solar Observatory

Planck ESA mission for microwave astronomy

RHESSI Reuven Ramaty High Energy Solar Spectroscopic Imager

ROSAT Röntgensatellit

Rosetta ESA mission to comet $67 \mathrm{P} /$ Churyumov-Gerasimenko 
RXTE Rossi X-ray Timing Explorer

SDO Solar Dynamics Observatory

Skylab NASA space station

SMEX Small Explorer Mission

$S M M$ Solar Maximum Mission

SNOE Student Nitric Oxide Explorer

SOHO Solar and Heliospheric Observatory

SORCE Solar Radiation and Climate Experiment

Spacelab Laboratory for use on Space Shuttle flights

SPARTAN Shuttle-launched satellites for solar studies

Spitzer Space Infrared Telescope Facility

STEREO Solar Terrestrial Relations Observatory

STS Space Transportation System

Suzaku Japanese X-ray astronomy mission, formerly Astro-E2

Swift NASA Gamma-Ray Burst Mission

$T D-1$ ESA UV mission

TIMED Thermosphere, Ionosphere and Mesosphere Energetics and Dynamics mission

TRACE Transition Region and Coronal Explorer

UARS Upper Atmosphere Research Satellite

Voyager NASA planetary and interstellar mission (two spacecraft, Voyager-1 and Voyager-2)

WFIRST Wide-Field Infrared Survey Telescope

WMAP Wilkinson Microwave Anisotropy Probe

$X M M$-Newton X-ray Multi-Mirror Mission

Yohkoh Solar X-ray observatory

\section{General}

2MASS Two Micron All Sky Survey

ACCESS Absolute Color Calibration Experiment for Standard Stars

ACIS Advanced CCD Imaging Spectrometer

ACRIM Active Cavity Radiometer Irradiance Monitor

ACS Advanced Camera for Surveys

ADS Astrophysics Data System

AIA Atmospheric Imaging Assembly

ALS Advanced Light Source

APS Active pixel sensor

ASM All-Sky Monitor

ATLAS Atmospheric Laboratory for Applications and Science, on the Space Shuttle

ATM Apollo Telescope Mount

BCS Bragg Crystal Spectrometer and bent crystal spectrometer

BESSY Berlin Electron Storage ring for Synchrotron radiation

BIPM Bureau International des Poids et Mesures

CALSPEC Calibration data base for the HST and the JWST, maintained by the STScI

CCD Charge-coupled device

CDS Coronal Diagnostic Spectrometer

CHIANTI An atomic database for spectroscopic diagnostics of astrophysical plasmas

CIPM Comité International des Poids et Mesures

CMB Cosmic Microwave Background

CME Coronal mass ejection

CNES Centre National d'Etudes Spatiales

COS Cosmic Origins Spectrograph 
CSL Centre Spatial de Liège

CTE Charge transfer efficiency

DIARAD Differential Absolute Radiometer

ECR Electrically calibrated radiometer

EGS Extreme-ultraviolet Grating Spectrograph

EIS EUV Imaging Spectrometer

EIT Extreme-ultraviolet Imaging Telescope

EPIC European Photon Imaging Camera

ESA European Space Agency

ESP EUV spectro-photometer

ESR Electrical substitution radiometer

EUNIS Extreme-Ultraviolet Normal Incidence Spectrograph

EUV Extreme ultraviolet

EUVI EUV Imager

EVE Extreme ultraviolet Variability Experiment

FIR Far infrared

FIRAS Far Infrared Absolute Spectrophotometer

FIS Far Infrared Surveyor

FOC Faint Object Camera

FOS Faint Object Spectrograph

FOV Field of view

FTS Fourier transform spectrometer

FUV Far ultraviolet

FWHM Full width at half maximum

GEANT Geometry and Tracking, a high-energy photon and particle transport code

GI Grazing incidence

GIS Grazing incidence spectrometer

GRBM Gamma-ray Burst Monitor

GSFC Goddard Space Flight Center

HEASARC High Energy Astrophysics Science Archive Research Center

HETG High Energy Transmission Grating

HEXTE High Energy X-ray Timing Experiment

HF Hickey-Frieden radiometer on NIMBUS-7

HFI High Frequency Instrument

HI Heliospheric Imager

HIFI Heterodyne Instrument for the Far Infrared

HMI Helioseismic and Magnetic Imager for $S D O$

HPGSPC High Pressure Gas Scintillator Proportional Counter

HRC High Resolution Camera

HRI High Resolution Imager

HRMA High Resolution Mirror Assembly

HRTS High Resolution Telescope and Spectrograph

HUT Hopkins Ultraviolet Telescope

HXD Hard X-ray Detector

HXT Hard X-ray Telescope

IACHEC International Astronomical Consortium for High Energy Calibration

IAS Institut d'Astrophysique Spatiale

IAU International Astronomical Union

IR Infrared 
IRC InfraRed Camera

ISAS Institute of Space and Astronautical Science, Japan

ISSI International Space Science Institute

JAXA Japan Aerospace Exploration Agency

KAO Kuiper Airborne Observatory

$\Lambda$ AMBDA Legacy Archive for Microwave Background Data Analysis

LASCO Large Angle Spectroscopic Coronagraph

LASP Laboratory for Atmospheric and Space Physics

LECS Low Energy Concentrator Spectrometer

LETG Low Energy Transmission Grating

LFI Low Frequency Instrument

LMSAL Lockheed Martin Solar and Astrophysics Laboratory

LTE Local thermodynamic equilibrium

MAST Mikulski Archive for Space Telescopes

MCP Microchannel plate

MECS Medium Energy Concentrator Spectrometer

MEGS Multiple EUV Grating Spectrograph

MIR Mid infrared

MIT Massachusetts Institute of Technology

MLS Metrology Light Source

MOS Metal oxide semiconductor

MPE Max-Planck-Institut für extraterrestrische Physik

MPG Max-Planck-Gesellschaft

MPS Max-Planck-Institut für Sonnensystemforschung, formerly Max-Planck-Institut für Aeronomie (MPAE)

MSFC Marshall Space Flight Center

MUV Medium ultraviolet

NASA National Aeronautics and Space Administration (US)

NBS National Bureau of Standards, now NIST

NI Normal incidence

NIR Near infrared

NIRSpec Near infrared multiobject dispersive spectrograph to be flown on the JWST

NIS Normal incidence spectrometer

NISP Near-infrared Spectrograph and Photometer on Euclid

NIST National Institute of Standards and Technology (US)

NLTE Non local thermodynamic equilibrium

NRL Naval Research Laboratory

NUV Near ultraviolet

PACS Photodetector Array Camera and Spectrometer for Herschel

PANTER X-ray test facility near München, Germany

PCA Proportional Counter Array

PDS Phoswich Detection System

PMOD/WRC Physikalisch-Meteorologisches Observatorium Davos / World Radiation Center

PSF Point spread function

PSI Paul Scherrer Institut

PSPC Position Sensitive Proportional Counters

PTB Physikalisch-Technische Bundesanstalt

QCM Quartz-crystal micro balance

QE Quantum efficiency 
RAL Rutherford Appleton Laboratory

RaMCaF Rainwater Memorial Calibration Facility

RCSS Radiometric calibration spectral source

RMC Rotation Modulation Collimator

SAA South Atlantic Anomaly

SAO Smithsonian Astrophysical Observatory

SARR Space absolute radiometric reference scale

SDD Silicon drift detector

SECCHI Sun Earth Connection Coronal and Heliospheric Investigation

SED Spectral energy distribution

SEE Solar EUV Experiment

SEM Solar Extreme-ultraviolet Monitor

SERTS Solar Extreme-ultraviolet Research Telescope and Spectrograph

SI Système International d'Unités, International System of Units

SIM Spectral Irradiance Monitor

SIRS Solar irradiance reference spectra

SM1 $(2,3,4)$ HST Servicing Missions

SN Supernova

SolACES Solar Auto-Calibrating EUV/UV Spectrophotometer

SOLSTICE Solar-Stellar Irradiance Comparison Experiment

SOT Solar Optical Telescope

SPIRE Spectral and Photometric Imaging Receiver

SSI Solar spectral irradiance

STIS Space Telescope Imaging Spectrograph

STScI Space Telescope Science Institute

SUMER Solar Ultraviolet Measurements of Emitted Radiation

SURF Synchrotron Ultraviolet Radiation Facility

SUSIM Solar Ultraviolet Spectral Irradiance Monitor

SXT Soft X-ray Telescope

TCF Telescope Calibration Facility (NIST)

TIM Total Irradiance Monitor

TSI Total solar irradiance

UKSA United Kingdom Space Agency

UV Ultraviolet

UVCS Ultraviolet Coronagraph Spectrometer

VIRGO Variability of Solar Irradiance and Gravity Oscillations

VUV Vacuum ultraviolet

WBS Wide Band Spectrometer

WD White Dwarf

WFC Wide Field Camera

XACT X-ray Astronomy Calibration and Testing, Palermo, Italy

XIS X-ray Imaging Spectrometer

XPS XUV Photometer System

XRCF X-ray and Cryogenics Facility, NASA MSFC

XRS X-ray Spectrometer

XRT X-ray Telescope

XUV Extreme ultraviolet 


\section{References}

Aalders JWG, van Duinen RJ, Luinge W, Wildeman KJ (1975) Calibration of the UV experiment in ANS. Space Science Instrumentation 1:343-350

Amati L, Cinti MN, Feroci M, Costa E, Frontera F, dal Fiume D, Collina P, Nicastro L, Orlandini M, Palazzi E, Rapisarda M, Zavattini G (1997) BeppoSAX GRBM on-ground calibration data analysis. In: Siegmund OH, Gummin MA (eds) EUV, X-Ray, and Gamma-Ray Instrumentation for Astronomy VIII, Society of Photo-Optical Instrumentation Engineers (SPIE) Conference Series, vol 3114, pp 176-185

Amendola L, Appleby S, Bacon D, Baker T, Baldi M, Bartolo N, Blanchard A, Bonvin C, Borgani S, Branchini E, Burrage C, Camera S, Carbone C, Casarini L, Cropper M, de Rham C, Di Porto C, Ealet A, Ferreira PG, Finelli F, García-Bellido J, Giannantonio T, Guzzo L, Heavens A, Heisenberg L, Heymans C, Hoekstra H, Hollenstein L, Holmes R, Horst O, Jahnke K, Kitching TD, Koivisto T, Kunz M, La Vacca G, March M, Majerotto E, Markovic K, Marsh D, Marulli F, Massey R, Mellier Y, Mota DF, Nunes N, Percival W, Pettorino V, Porciani C, Quercellini C, Read J, Rinaldi M, Sapone D, Scaramella R, Skordis C, Simpson F, Taylor A, Thomas S, Trotta R, Verde L, Vernizzi F, Vollmer A, Wang Y, Weller J, Zlosnik T (2013) Cosmology and Fundamental Physics with the Euclid Satellite. Living Reviews in Relativity 16:6, DOI 10.12942/lrr-2013-6, 1206.1225

Arimatsu K, Doi Y, Wada T, Takita S, Kawada M, Matsuura S, Ootsubo T, Kataza H (2014) Point source calibration of the AKARI/FIS all-sky survey maps for stacking analysis. PASJ66:47, DOI $10.1093 / \mathrm{pasj} / \mathrm{psu010,} 1403.2772$

Arnold D, Ulm G (1994) Determination of photon emission probabilities of radionuclides using a $\mathrm{Si}(\mathrm{Li})$ detector calibrated by the primary standard source, BESSY. Nuclear Instruments and Methods in Physics Research A 339:43-48, DOI 10.1016/0168-9002(94)91776-0

Arp U, Clark CW, Farrell AP, Fein E, Furst ML, Hagley EW (2002) Synchrotron ultraviolet radiation facility SURF III. Review of Scientific Instruments 73:1674-1676, DOI 10.1063/1.1445833

Arvesen JC, Griffin RN Jr, Pearson BD Jr (1969) Determination of extraterrestrial solar spectral irradiance from a research aircraft. Appl. Opt.8:2215-2232, DOI 10.1364/AO.8.002215

Aschenbach B, Bräuninger H, Briel U, Brinkmann W, Fink H, Heinecke N, Hippmann H, Kettenring G, Metzner G, Ondrusch A (1981) The ROSAT mission. Space Sci. Rev.30:569-573, DOI 10.1007/BF01246075

Aumann HH, Beichman CA, Gillett FC, de Jong T, Houck JR, Low FJ, Neugebauer G, Walker RG, Wesselius PR (1984) Discovery of a shell around Alpha Lyrae. ApJ278:L23-L27, DOI $10.1086 / 184214$

Bailey SM, Woods TN, Barth CA, Solomon SC (1999) Measurements of the solar soft X-ray irradiance from the Student Nitric Oxide Explorer. Geophys. Res. Lett.26:1255-1258, DOI 10.1029/1999GL900236

Ballester P, Rosa MR (1997) Modeling echelle spectrographs. A\&AS126:563-571, DOI 10.1051/aas: 1997283

Balog Z, Müller T, Nielbock M, Altieri B, Klaas U, Blommaert J, Linz H, Lutz D, Moór A, Billot N, Sauvage M, Okumura K (2014) The Herschel-PACS photometer calibration. Point-source flux calibration for scan maps. Experimental Astronomy 37:129-160, DOI 10.1007/s10686-013-9352-3, 1309.6099

Barnstedt J, Kappelmann N, Appenzeller I, Fromm A, Gölz M, Grewing M, Gringel W, Haas C, Hopfensitz W, Krämer G, Krautter J, Lindenberger A, Mandel H, Widmann H (1999) The ORFEUS II Echelle Spectrometer: Instrument description, performance and data reduction. A\&AS134:561-567, DOI 10.1051/aas:1999156, astro-ph/0006295

Bautz MW, Pivovaroff MJ, Kissel SE, Prigozhin GY, Isobe T, Jones SE, Ricker GR, Thornagel R, Kraft S, Scholze F, Ulm G (2000) Absolute calibration of ACIS x-ray CCDs using calculable 
undispersed synchrotron radiation. In: Truemper JE, Aschenbach B (eds) X-Ray Optics, Instruments, and Missions III, Society of Photo-Optical Instrumentation Engineers (SPIE) Conference Series, vol 4012, pp 53-67

BenMoussa A, Gissot S, Schühle U, Del Zanna G, Auchère F, Mekaoui S, Jones AR, Walton D, Eyles CJ, Thuillier G, Seaton D, Dammasch IE, Cessateur G, Meftah M, Andretta V, Berghmans D, Bewsher D, Bolsée D, Bradley L, Brown DS, Chamberlin PC, Dewitte S, Didkovsky LV, Dominique M, Eparvier FG, Foujols T, Gillotay D, Giordanengo B, Halain JP, Hock RA, Irbah A, Jeppesen C, Judge DL, Kretzschmar M, McMullin DR, Nicula B, Schmutz W, Ucker G, Wieman S, Woodraska D, Woods TN (2013) On-Orbit Degradation of Solar Instruments. Sol. Phys.288:389-434, DOI 10.1007/s11207-013-0290-z, 1304.5488

BenMoussa A, Giordanengo B, Gissot S, Dammasch IE, Dominique M, Hochedez JF, Soltani A, Bourzgui N, Saito T, Schühle U, Gottwald A, Kroth U, Jones AR (2015) Degradation assessment of LYRA after 5 years on orbit - Technology Demonstration -. Experimental Astronomy 39:29-43, DOI 10.1007/s10686-014-9437-7

Bewsher D, Brown DS, Eyles CJ, Kellett BJ, White GJ, Swinyard B (2010) Determination of the Photometric Calibration and Large-Scale Flatfield of the STEREO Heliospheric Imagers: I. HI-1. Sol. Phys.264:433-460, DOI 10.1007/s11207-010-9582-8

Bewsher D, Brown DS, Eyles CJ (2012) Long-Term Evolution of the Photometric Calibration of the STEREO Heliospheric Imagers: I. HI-1. Sol. Phys.276:491-499, DOI 10.1007/s11207-011-9874-7

Blackwell DE (1975) The Presidential Address: Uncertainty in Astronomy. QJRAS16:361-377

Blackwell DE, Leggett SK, Petford AD, Mountain CM, Selby MJ (1983) Absolute calibration of the infrared flux from VEGA at 1.24, 2.20, 3.76 and 4.6 microns by comparison with a standard furnace. MNRAS205:897-905

Blades JC, Osmer SJ (1994) Calibrating Hubble Space Telescope, Proceedings of a Workshop. In: Bulletin of the American Astronomical Society, Bulletin of the American Astronomical Society, vol 26, p 1212

Bless RC, Code AD, Fairchild ET (1976) Ultraviolet photometry from the Orbiting Astronomical Observatory. XXI - Absolute energy distribution of stars in the ultraviolet. ApJ203:410-416, DOI 10.1086/154092

Boella G, Chiappetti L, Conti G, Cusumano G, del Sordo S, La Rosa G, Maccarone MC, Mineo T, Molendi S, Re S, Sacco B, Tripiciano M (1997) The medium-energy concentrator spectrometer on board the BeppoSAX X-ray astronomy satellite. A\&AS122:327-340, DOI 10.1051/aas:1997138

Boerner P, Edwards C, Lemen J, Rausch A, Schrijver C, Shine R, Shing L, Stern R, Tarbell T, Title A, Wolfson CJ, Soufli R, Spiller E, Gullikson E, McKenzie D, Windt D, Golub L, Podgorski W, Testa P, Weber M (2012) Initial Calibration of the Atmospheric Imaging Assembly (AIA) on the Solar Dynamics Observatory (SDO). Sol. Phys.275:41-66, DOI 10.1007/s11207-011-9804-8

Boerner PF, Testa P, Warren H, Weber MA, Schrijver CJ (2014) Photometric and Thermal Cross-calibration of Solar EUV Instruments. Sol. Phys.289:2377-2397, DOI 10.1007/ s11207-013-0452-z, 1307.8045

Boggess A, Carr FA, Evans DC, Fischel D, Freeman HR, Fuechsel CF, Klinglesmith DA, Krueger VL, Longanecker GW, Moore JV (1978) The IUE spacecraft and instrumentation. Nature275:372-377, DOI 10.1038/275372a0

Bohlin JD, Frost KJ, Burr PT, Guha AK, Withbroe GL (1980) Solar Maximum Mission. Sol. Phys.65:5-14, DOI 10.1007/BF00151380

Bohlin RC (2007) HST Stellar Standards with 1\% Accuracy in Absolute Flux. In: Sterken C (ed) The Future of Photometric, Spectrophotometric and Polarimetric Standardization, Astronomical Society of the Pacific Conference Series, vol 364, p 315, astro-ph/0608715

Bohlin RC (2014) Hubble Space Telescope CALSPEC Flux Standards: Sirius (and Vega). AJ147:127, DOI 10.1088/0004-6256/147/6/127 
Bohlin RC, Frimout D, Lillie CF (1974) Absolute fix measurements in the rocket ultraviolet. A\&A30:127-134

Bohlin RC, Harris AW, Holm AV, Gry C (1990) The ultraviolet calibration of the Hubble Space Telescope. IV - Absolute IUE fluxes of Hubble Space Telescope standard stars. ApJS73:413-439, DOI 10.1086/191474

Bohlin RC, Colina L, Finley DS (1995) White Dwarf Standard Stars: G191-B2B, GD 71, GD 153, HZ 43. AJ110:1316-1325, DOI 10.1086/117606

Bohlin RC, Gordon KD, Tremblay PE (2014) Techniques and Review of Absolute Flux Calibration from the Ultraviolet to the Mid-Infrared. PASP126:711-732, DOI 10.1086/677655, 1406.1707

Böhm T, Holschneider M, Lignières F, Petit P, Rainer M, Paletou F, Wade G, Alecian E, Carfantan H, Blazère A, Mirouh GM (2015) Discovery of starspots on Vega. First spectroscopic detection of surface structures on a normal A-type star. A\&A577:A64, DOI 10.1051/0004-6361/201425425, 1411.7789

Booth AJ, Selby MJ, Blackwell DE, Petford AD, Arribas S (1989) Determination of the absolute flux from VEGA at 2.250 microns. A\&A218:167-168

Bottema M (1993) Reflective correctors for the Hubble Space Telescope axial instruments. Appl. Opt.32:1768-1774, DOI 10.1364/AO.32.001768

Bowen PJ, Bowles JA, Glencross WM, Speer RJ, Timothy AF, Timothy JG, Willmore AP (1971) A grazing incidence monochromator for satellite studies of the solar He II Lyman-alpha line at 303.8 A. Appl. Opt.10:28-33, DOI 10.1364/AO.10.000028

Bowyer S, Malina RF (1991) The extreme ultraviolet explorer mission. Advances in Space Research 11:205-215, DOI 10.1016/0273-1177(91)90077-W

Bradt HV, Rothschild RE, Swank JH (1993) X-ray timing explorer mission. A\&AS97:355-360

Brejnholt NF, Christensen FE, Jakobsen AC, Hailey CJ, Koglin JE, Blaedel KL, Stern M, Thornhill D, Sleator C, Zhang S, Craig WW, Madsen KK, Decker T, Pivovaroff MJ, Vogel JK (2011) NuSTAR ground calibration: The Rainwater Memorial Calibration Facility (RaMCaF). In: Society of Photo-Optical Instrumentation Engineers (SPIE) Conference Series, Society of Photo-Optical Instrumentation Engineers (SPIE) Conference Series, vol 8147, p 0, DOI 10.1117/12.894659

Brejnholt NF, Christensen FE, Westergaard NJ, Hailey CJ, Koglin JE, Craig WW (2012) NuSTAR on-ground calibration: II. Effective area. In: Society of Photo-Optical Instrumentation Engineers (SPIE) Conference Series, Society of Photo-Optical Instrumentation Engineers (SPIE) Conference Series, vol 8443, p 1, DOI 10.1117/12.925631

Brekke P, Thompson WT, Woods TN, Eparvier FG (2000) The Extreme-Ultraviolet Solar Irradiance Spectrum Observed with the Coronal Diagnostic Spectrometer (CDS) on SOHO. ApJ536:959-970, DOI 10.1086/308966

Bridges JM, Ott WR (1977) Vacuum ultraviolet radiometry. III - The argon mini-arc as a new secondary standard of spectral radiance. Appl. Opt.16:367-376, DOI 10.1364/AO.16.000367

Briel UG, B A, Hasinger G, Hippmann H, Pfeffermann E, Predehl P, Schmitt JHMM, Schwentker O, Voges W, Zimmermann U, George IM, Snowden SL, Turner TJ, David FRJ L Harnden, Kearns KE, Zombeck MV, Barstow MA, Osborne JP, Pye JP, Watson M, West RG, Willingale R (1997) ROSAT User's Handbook. Max-Planck-Institut für extraterrestrische Physik, Garching, D, 1st edn, http://heasarc.gsfc.nasa.gov/docs/rosat/ruh/handbook/handbook.html

Broadfoot AL, Sandel BR, Shemansky DE, Atreya SK, Donahue TM, Moos HW, Bertaux JL, Blamont JE, Ajello JM, Strobel DF (1977) Ultraviolet spectrometer experiment for the Voyager mission. Space Sci. Rev.21:183-205, DOI 10.1007/BF00200850

Brown CM, Feldman U, Seely JF, Korendyke CM, Hara H (2008) Wavelengths and Intensities of Spectral Lines in the 171-211 and 245-291 A Ranges from Five Solar Regions Recorded by the Extreme-Ultraviolet Imaging Spectrometer (EIS) on Hinode. ApJS176:511-535, DOI $10.1086 / 529378$ 
Brown DS, Bewsher D, Eyles CJ (2009) Calibrating the Pointing and Optical Parameters of the STEREO Heliospheric Imagers. Sol. Phys.254:185-225, DOI 10.1007/s11207-008-9277-6

Brueckner GE, Edlow KL, Floyd LE IV, Lean JL, VanHoosier ME (1993) The solar ultraviolet spectral irradiance monitor (SUSIM) experiment on board the Upper Atmosphere Research Satellite (UARS). J. Geophys. Res.98:10,695-10,711, DOI 10.1029/93JD00410

Brueckner GE, Howard RA, Koomen MJ, Korendyke CM, Michels DJ, Moses JD, Socker DG, Dere KP, Lamy PL, Llebaria A, Bout MV, Schwenn R, Simnett GM, Bedford DK, Eyles CJ (1995) The Large Angle Spectroscopic Coronagraph (LASCO). Sol. Phys.162:357-402, DOI 10.1007/BF00733434

Burlov-Vasiljev KA, Gurtovenko EA, Matvejev YB (1995) New absolute measurements of the solar spectrum. Sol. Phys.157:51-73, DOI 10.1007/BF00680609

Butkovskaya V, Plachinda S, Valyavin G, Baklanova D, Lee BC (2011) The long-term variability of Vega. Astronomische Nachrichten 332:956-960, DOI 10.1002/asna.201111587

Butkovskaya VV (2014) On the variability of Vega. Bulletin Crimean Astrophysical Observatory 110:80-84, DOI 10.3103/S0190271714010100

Canfield LR, Vest RE, Korde R, Schmidtke H, Desor R (1998) Absolute silicon photodiodes for $160 \mathrm{~nm}$ to $254 \mathrm{~nm}$ photons. Metrologia 35:329, DOI 10.1088/0026-1394/35/4/19

Carlson RW, Ogawa HS, Phillips E, Judge DL (1984) Absolute measurement of the extreme uv solar flux. Appl Opt 23(14):2327-2332, DOI 10.1364/AO.23.002327, URL http://ao.osa.org/abstract. cfm?URI=ao-23-14-2327

Chipman EG (1981) The Solar Maximum Mission. ApJ244:L113-L115, DOI 10.1086/183493

Clette F, Hochedez JF, Newmark JS, Moses JD, Auchère F, Defise JM, Delaboudinière JP (2002) The Radiometric Calibration of the Extreme Ultraviolet Imaging Telescope. ISSI Scientific Reports Series 2:121-134

Code AD (1960) Stellar Energy Distribution. In: Greenstein JL (ed) Stellar Atmospheres, pp 50-87

Code AD, Houck TE, McNall JF, Bless RC, Lillie CF (1970) Ultraviolet Photometry from the Orbiting Astronomical Observatory. I. Instrumentation and Operation. ApJ161:377-388, DOI 10.1086/150545

Cohen M, Walker RG, Barlow MJ, Deacon JR (1992a) Spectral irradiance calibration in the infrared. I - Ground-based and IRAS broadband calibrations. AJ104:1650-1657, DOI 10.1086/ 116349

Cohen M, Walker RG, Witteborn FC (1992b) Spectral irradiance calibration in the infrared. II - Alpha Tau and the recalibration of the IRAS low resolution spectrometer. AJ104:2030-2044, DOI 10.1086/116379

Cohen M, Walker RG, Jayaraman S, Barker E, Price SD (2001) Spectral Irradiance Calibration in the Infrared. XII. Radiometric Measurements from the Midcourse Space Experiment. AJ121:1180-1191, DOI 10.1086/318751

Cohen M, Wheaton WA, Megeath ST (2003) Spectral Irradiance Calibration in the Infrared. XIV. The Absolute Calibration of 2MASS. AJ126:1090-1096, DOI 10.1086/376474, astro-ph/0304350

Colina L, Bohlin RC (1994) Absolute flux calibration of optical spectrophotometric standard stars. AJ108:1931-1935, DOI 10.1086/117206

Conard SJ, Barkhouser RH, Evans JP, Friedman SD, Kruk JW, Moos HW, Ohl RG, Sahnow DJ (2000) Far Ultraviolet Spectroscopic Explorer optical system: lessons learned. In: Fineschi S, Korendyke CM, Siegmund $\mathrm{OH}$, Woodgate BE (eds) Instrumentation for UV/EUV Astronomy and Solar Missions, Society of Photo-Optical Instrumentation Engineers (SPIE) Conference Series, vol 4139, pp 186-198

Cook AH (1994) The observational foundations of physics. Cambridge University Press, New York

Crommelynck D, Fichot A, Lee RB III, Romero J (1995) First realisation of the Space Absolute Radiometric Reference (SARR) during the ATLAS 2 flight period. Advances in Space Research 
16:17-23, DOI 10.1016/0273-1177(95)00261-C

Culhane JL, Harra LK, James AM, Al-Janabi K, Bradley LJ, Chaudry RA, Rees K, Tandy JA, Thomas P, Whillock MCR, Winter B, Doschek GA, Korendyke CM, Brown CM, Myers S, Mariska J, Seely J, Lang J, Kent BJ, Shaughnessy BM, Young PR, Simnett GM, Castelli CM, Mahmoud S, Mapson-Menard H, Probyn BJ, Thomas RJ, Davila J, Dere K, Windt D, Shea J, Hagood R, Moye R, Hara H, Watanabe T, Matsuzaki K, Kosugi T, Hansteen V, Wikstol $\varnothing ~(2007)$ The EUV Imaging Spectrometer for Hinode. Sol. Phys.243:19-61, DOI 10.1007/s01007-007-0293-1

Danzmann K, Günther M, Fischer J, Kühne M, Kock M (1988) High current hollow cathode as a radiometric transfer standard source for the extreme vacuum ultraviolet. Appl. Opt.27:49474951, DOI 10.1364/AO.27.004947

Davidsen AF, Long KS, Durrance ST, Blair WP, Bowers CW, Conard SJ, Feldman PD, Ferguson HC, Fountain GH, Kimble RA, Kriss GA, Moos HW, Potocki KA (1992) The Hopkins Ultraviolet Telescope - Performance and calibration during the Astro-1 mission. ApJ392:264-271, DOI 10.1086/171424

De Pontieu B, Title AM, Lemen JR, Kushner GD, Akin DJ, Allard B, Berger T, Boerner P, Cheung M, Chou C, Drake JF, Duncan DW, Freeland S, Heyman GF, Hoffman C, Hurlburt NE, Lindgren RW, Mathur D, Rehse R, Sabolish D, Seguin R, Schrijver CJ, Tarbell TD, Wülser JP, Wolfson CJ, Yanari C, Mudge J, Nguyen-Phuc N, Timmons R, van Bezooijen R, Weingrod I, Brookner R, Butcher G, Dougherty B, Eder J, Knagenhjelm V, Larsen S, Mansir D, Phan L, Boyle P, Cheimets PN, DeLuca EE, Golub L, Gates R, Hertz E, McKillop S, Park S, Perry T, Podgorski WA, Reeves K, Saar S, Testa P, Tian H, Weber M, Dunn C, Eccles S, Jaeggli SA, Kankelborg CC, Mashburn K, Pust N, Springer L, Carvalho R, Kleint L, Marmie J, Mazmanian E, Pereira TMD, Sawyer S, Strong J, Worden SP, Carlsson M, Hansteen VH, Leenaarts J, Wiesmann M, Aloise J, Chu KC, Bush RI, Scherrer PH, Brekke P, Martinez-Sykora J, Lites BW, McIntosh SW, Uitenbroek H, Okamoto TJ, Gummin MA, Auker G, Jerram P, Pool P, Waltham N (2014) The Interface Region Imaging Spectrograph (IRIS). Sol. Phys.289:2733-2779, DOI 10.1007/s11207-014-0485-y, 1401.2491

de Vries CP, den Herder JW, Gabriel C, Gonzalez-Riestra R, Ibarra A, Kaastra JS, Pollock AMT, Raassen AJJ, Paerels FBS (2015) Calibration and in-orbit performance of the reflection grating spectrometer onboard XMM-Newton. A\&A573:A128, DOI 10.1051/0004-6361/201423704, 1410. 5251

Defise JM, Song XY, Delaboudiniere JP, Artzner GE, Carabetian C, Hochedez JFE, Brunaud J, Moses JD, Catura RC, Clette F, Maucherat AJ (1995) Calibration of the EIT instrument for the SOHO mission. In: Fineschi S (ed) X-Ray and EUV/FUV Spectroscopy and Polarimetry, Society of Photo-Optical Instrumentation Engineers (SPIE) Conference Series, vol 2517, pp 29-39

Del Zanna G, Bromage BJI, Landi E, Landini M (2001) Solar EUV spectroscopic observations with SOHO/CDS. I. An in-flight calibration study. A\&A379:708-734, DOI 10.1051/0004-6361: 20011220

Del Zanna G, Andretta V, Chamberlin PC, Woods TN, Thompson WT (2010) The EUV spectrum of the Sun: long-term variations in the SOHO CDS NIS spectral responsivities. A\&A518:A49, DOI 10.1051/0004-6361/200912904

Delaboudinière JP, Artzner GE, Brunaud J, Gabriel AH, Hochedez JF, Millier F, Song XY, Au B, Dere KP, Howard RA, Kreplin R, Michels DJ, Moses JD, Defise JM, Jamar C, Rochus P, Chauvineau JP, Marioge JP, Catura RC, Lemen JR, Shing L, Stern RA, Gurman JB, Neupert WM, Maucherat A, Clette F, Cugnon P, van Dessel EL (1995) EIT: Extreme-Ultraviolet Imaging Telescope for the SOHO Mission. Sol. Phys.162:291-312, DOI 10.1007/BF00733432

den Herder JW, Brinkman AC, Kahn SM, Branduardi-Raymont G, Thomsen K, Aarts H, Audard M, Bixler JV, den Boggende AJ, Cottam J, Decker T, Dubbeldam L, Erd C, Goulooze H, 
Güdel M, Guttridge P, Hailey CJ, Janabi KA, Kaastra JS, de Korte PAJ, van Leeuwen BJ, Mauche C, McCalden AJ, Mewe R, Naber A, Paerels FB, Peterson JR, Rasmussen AP, Rees K, Sakelliou I, Sako M, Spodek J, Stern M, Tamura T, Tandy J, de Vries CP, Welch S, Zehnder A (2001) The Reflection Grating Spectrometer on board XMM-Newton. A\&A365:L7-L17, DOI 10.1051/0004-6361:20000058

Didkovsky L, Judge D, Wieman S, Woods T, Jones A (2012) EUV SpectroPhotometer (ESP) in Extreme Ultraviolet Variability Experiment (EVE): Algorithms and Calibrations. Sol. Phys.275:179-205, DOI 10.1007/s11207-009-9485-8, 0911.0722

Dixon WV, Blair WP, Kruk JW, Romelfanger ML (2013) The Hopkins Ultraviolet Telescope: The Final Archive. PASP125:431-443, DOI 10.1086/670227, 1303.6131

Dudok de Wit T (2011) A method for filling gaps in solar irradiance and solar proxy data. A\&A533:A29, DOI 10.1051/0004-6361/201117024

Dudok de Wit T (2014) Some Regression Problems in Solar-Terrestrial Sciences: Learning from Mistakes. In: Fraix-Burnet D (ed) EAS Publications Series, EAS Publications Series, vol 66, pp 77-87, DOI 10.1051/eas/1466007

Engelke CW, Price SD, Kraemer KE (2006) Spectral Irradiance Calibration in the Infrared. XVI. Improved Accuracy in the Infrared Spectra of the Secondary and Tertiary Standard Calibration Stars. AJ132:1445-1463, DOI 10.1086/505865

Engelke CW, Price SD, Kraemer KE (2010) Spectral Irradiance Calibration in the Infrared. XVII. Zero-magnitude Broadband Flux Reference for Visible-to-infrared Photometry. AJ140:19191928, DOI 10.1088/0004-6256/140/6/1919

European Space Agency (1994) Horizon 2000 Plus - European Space Science in the 21st Century. Tech. rep., ESA, ESA SP-1180

Fazio GG, Hora JL, Allen LE, Ashby MLN, Barmby P, Deutsch LK, Huang JS, Kleiner S, Marengo M, Megeath ST, Melnick GJ, Pahre MA, Patten BM, Polizotti J, Smith HA, Taylor RS, Wang Z, Willner SP, Hoffmann WF, Pipher JL, Forrest WJ, McMurty CW, McCreight CR, McKelvey ME, McMurray RE, Koch DG, Moseley SH, Arendt RG, Mentzell JE, Marx CT, Losch P, Mayman P, Eichhorn W, Krebs D, Jhabvala M, Gezari DY, Fixsen DJ, Flores J, Shakoorzadeh K, Jungo R, Hakun C, Workman L, Karpati G, Kichak R, Whitley R, Mann S, Tollestrup EV, Eisenhardt P, Stern D, Gorjian V, Bhattacharya B, Carey S, Nelson BO, Glaccum WJ, Lacy M, Lowrance PJ, Laine S, Reach WT, Stauffer JA, Surace JA, Wilson G, Wright EL, Hoffman A, Domingo G, Cohen M (2004) The Infrared Array Camera (IRAC) for the Spitzer Space Telescope. ApJS154:10-17, DOI 10.1086/422843, astro-ph/0405616

Finley DS, Basri G, Bowyer S (1984) Self-consistent recalibration of IUE determination of hot DA white dwarf effective temperatures. In: Mead JM, Chapman RD, Kondo Y (eds) NASA Conference Publication, NASA Conference Publication, vol 2349, pp 277-280

Finley DS, Basri G, Bowyer S (1990) The temperature scale of hot DA white dwarfs - Temperatures from far-ultraviolet continuum fluxes. ApJ359:483-498, DOI 10.1086/169080

Fleck B, Domingo V, Poland AI (1995) The SOHO mission. Sol. Phys.162:XII+531

Foukal PV, Hoyt C, Kochling H, Miller P (1990) Cryogenic absolute radiometers as laboratory irradiance standards, remote sensing detectors, and pyroheliometers. Appl Opt 29(7):988-993, DOI 10.1364/AO.29.000988, URL http://ao.osa.org/abstract.cfm?URI=ao-29-7-988

Fox NP, Martin JE (1990) Comparison of two cryogenic radiometers by determining the absolute spectral responsivity of silicon photodiodes with an uncertainty of $0.02 \%$. Appl Opt 29(31):46864693, DOI 10.1364/AO.29.004686, URL http://ao.osa.org/abstract.cfm?URI=ao-29-31-4686

Frazin RA, Romoli M, Kohl JL, Gardner LD, Wang D, Howard RA, Kucera TA (2002) White Light Intercalibrations of UVCS, LASCO-C2 and Spartan 201/WLC. ISSI Scientific Reports Series 2:249-263 
Freyberg MJ, Bräuninger H, Burkert W, Hartner GD, Citterio O, Mazzoleni F, Pareschi G, Spiga D, Romaine S, Gorenstein P, Ramsey BD (2005) The MPE X-ray test facility PANTER: Calibration of hard X-ray (15-50 kev) optics. Experimental Astronomy 20:405-412, DOI 10.1007/s10686-006-9068-8

Fröhlich C (2013) Solar radiometry. In: Huber M, Pauluhn A, Culhane J, Timothy J, Wilhelm K, Zehnder A (eds) Observing Photons in Space, Springer, New York, Heidelberg, Dordrecht, London, pp 565-581

Fröhlich C (2015a) Degradation of radiometers in space: Application to VIRGO TSI. ftp://ftp. pmodwrc.ch/pub/Claus/VIRGO-TSI/VIRGO_TSI-vers64.pdf, to be submitted to Metrologia

Fröhlich C (2015b) VIRGO Radiometry: Update of the Characterization. ftp://ftp.pmodwrc.ch/ pub/Claus/VIRGO-TSI/VIRGO_2CharSpace.pdf to be submitted to Metrologia

Fröhlich C, Lean J (1998) Total solar irradiance variations: The construction of a composite and its comparison with models. In: Deubner FL, Christensen-Dalsgaard J, Kurtz D (eds) IAU Symposium 185: New Eyes to See Inside the Sun and Stars, Kluwer Academic Publ., Dordrecht, The Netherlands, pp 89-102

Fröhlich C, Crommelynck DA, Wehrli C, Anklin M, Dewitte S, Fichot A, Finsterle W, Jiménez A, Chevalier A, Roth H (1997) In-Flight Performance of the Virgo Solar Irradiance Instruments on SOHO. Sol. Phys.175:267-286, DOI 10.1023/A:1004929108864

Fulton T, Hopwood R, Baluteau JP, Benielli D, Imhof P, Lim T, Lu N, Marchili N, Naylor D, Polehampton E, Swinyard B, Valtchanov I (2014) Herschel SPIRE FTS relative spectral response calibration. Experimental Astronomy 37:381-395, DOI 10.1007/s10686-013-9364-z, 1401.2049

Gardner LD, Kohl JL, Daigneau PS, Dennis EF, Fineschi S, Michels J, Nystrom GU, Panasyuk A, Raymond JC, Reisenfeld DJ, Smith PL, Strachan L, Suleiman R, Noci GC, Romoli M, Ciaravella A, Modigliani A, Huber MC, Antonucci E, Benna C, Giordano S, Tondello G, Nicolosi P, Naletto G, Pernechele C, Spadaro D, Siegmund OH, Allegra A, Carosso PA, Jhabvala MD (1996) Stray light, radiometric, and spectral characterization of UVCS/SOHO: laboratory calibration and flight performance. In: Huffman RE, Stergis CG (eds) Ultraviolet Atmospheric and Space Remote Sensing: Methods and Instrumentation, Society of Photo-Optical Instrumentation Engineers (SPIE) Conference Series, vol 2831, pp 2-24

Gardner LD, Atkins N, Fineschi S, Smith PL, Kohl JL, Maccari L, Romoli M (2000) Efficiency variations of UVCS/SOHO based on laboratory measurements of replica gratings. In: Fineschi S, Korendyke CM, Siegmund OH, Woodgate BE (eds) Instrumentation for UV/EUV Astronomy and Solar Missions, Society of Photo-Optical Instrumentation Engineers (SPIE) Conference Series, vol 4139, pp 362-369

Gardner LD, Smith PL, Kohl JL, Atkins N, Ciaravella A, Miralles MP, Panasyuk A, Raymond JC, Strachan L Jr, Suleiman RM, Romoli M, Fineschi S (2002) UV Radiometric Calibration of UVCS. In: Pauluhn A, Huber M, von Steiger R (eds) The Radiometric Calibration of SOHO, vol 2, ESA, Noordwijk, pp 161-180, ISSI Scientific Report SR-002

Giacconi R, Kellogg E, Gorenstein P, Gursky H, Tananbaum H (1971) An X-Ray Scan of the Galactic Plane from UHURU. ApJ165:L27-L35, DOI 10.1086/180711

Goebel R, Yilmaz S, Köhler R (1996) Stability under vacuum of silicon trap detectors and their use as transfer instruments in cryogenic radiometry. Appl. Opt.35:4404-4407, DOI 10.1364/AO. 35.004404

Golub L, Deluca E, Austin G, Bookbinder J, Caldwell D, Cheimets P, Cirtain J, Cosmo M, Reid P, Sette A, Weber M, Sakao T, Kano R, Shibasaki K, Hara H, Tsuneta S, Kumagai K, Tamura T, Shimojo M, McCracken J, Carpenter J, Haight H, Siler R, Wright E, Tucker J, Rutledge H, Barbera M, Peres G, Varisco S (2007) The X-Ray Telescope (XRT) for the Hinode Mission. Sol. Phys.243:63-86, DOI 10.1007/s11207-007-0182-1 
Gondoin P, Aschenbach BR, Bräuninger H, de Chambure D, Collette JP, Egger R, van Katwijk K, Lumb DH, Peacock AJ, Stockman Y, Tock JP, Willingale R (1996) X-ray performance of a qualification model of an XMM mirror module. In: Siegmund OH, Gummin MA (eds) EUV, X-Ray, and Gamma-Ray Instrumentation for Astronomy VII, Society of Photo-Optical Instrumentation Engineers (SPIE) Conference Series, vol 2808, pp 390-401

Gondoin P, Aschenbach BR, Beijersbergen MW, Egger R, Jansen FA, Stockman Y, Tock JP (1998) Calibration of the first XXM flight mirror module: II. Effective area. In: Hoover RB, Walker AB (eds) X-Ray Optics, Instruments, and Missions, Society of Photo-Optical Instrumentation Engineers (SPIE) Conference Series, vol 3444, pp 290-301

Gottwald A, Klein R, Krumrey M, Müller P, Paustian W, Reichel T, Scholze F, Thornagel R (2014) Radiometrische Charakterisierung von Weltrauminstrumentierung. Sonderdruck aus PTB-Mitteilungen 3,4

Greenstein JL, Oke JB (1979) Ultraviolet spectrophotometry of degenerate stars. ApJ229:L141L144, DOI 10.1086/182948

Grewing M, Appenzeller I, Barnstedt J, Bowyer S, Hurwitz M, Krämer G, Kappelmann N, Krautter J, Mandel H (1998) ORFEUS. In: Wamsteker W, Gonzalez Riestra R, Harris B (eds) Ultraviolet Astrophysics Beyond the IUE Final Archive, ESA Special Publication, vol 413, p 757

Griffin M, Ade P (2013) Narrow-band imaging by use of interferometers. In: Huber M, Pauluhn A, Culhane J, Timothy J, Wilhelm K, Zehnder A (eds) Observing Photons in Space, Springer, New York, Heidelberg, Dordrecht, London, pp 333-348

Güver T, Özel F, Marshall H, Psaltis D, Guainazzi M, Díaz-Trigo M (2015) Systematic Uncertainties in the Spectroscopic Measurements of Neutron-Star Masses and Radii from Thermonuclear X-ray Bursts. III. Absolute Flux Calibration. ArXiv e-prints 1501.05330

Handy BN, Bruner ME, Tarbell TD, Title AM, Wolfson CJ, Laforge MJ, Oliver JJ (1998) UV Observations with the Transition Region and Coronal Explorer. Sol. Phys.183:29-43, DOI 10. 1023/A:1005079622620

Handy BN, Acton LW, Kankelborg CC, Wolfson CJ, Akin DJ, Bruner ME, Caravalho R, Catura RC, Chevalier R, Duncan DW, Edwards CG, Feinstein CN, Freeland SL, Friedlaender FM, Hoffmann CH, Hurlburt NE, Jurcevich BK, Katz NL, Kelly GA, Lemen JR, Levay M, Lindgren RW, Mathur DP, Meyer SB, Morrison SJ, Morrison MD, Nightingale RW, Pope TP, Rehse RA, Schrijver CJ, Shine RA, Shing L, Strong KT, Tarbell TD, Title AM, Torgerson DD, Golub L, Bookbinder JA, Caldwell D, Cheimets PN, Davis WN, Deluca EE, McMullen RA, Warren HP, Amato D, Fisher R, Maldonado H, Parkinson C (1999) The transition region and coronal explorer. Sol. Phys.187:229-260, DOI 10.1023/A:1005166902804

Harrison FA, Craig WW, Christensen FE, Hailey CJ, Zhang WW, Boggs SE, Stern D, Cook WR, Forster K, Giommi P, Grefenstette BW, Kim Y, Kitaguchi T, Koglin JE, Madsen KK, Mao PH, Miyasaka H, Mori K, Perri M, Pivovaroff MJ, Puccetti S, Rana VR, Westergaard NJ, Willis J, Zoglauer A, An H, Bachetti M, Barrière NM, Bellm EC, Bhalerao V, Brejnholt NF, Fuerst F, Liebe CC, Markwardt CB, Nynka M, Vogel JK, Walton DJ, Wik DR, Alexander DM, Cominsky LR, Hornschemeier AE, Hornstrup A, Kaspi VM, Madejski GM, Matt G, Molendi S, Smith DM, Tomsick JA, Ajello M, Ballantyne DR, Baloković M, Barret D, Bauer FE, Blandford RD, Brandt WN, Brenneman LW, Chiang J, Chakrabarty D, Chenevez J, Comastri A, Dufour F, Elvis M, Fabian AC, Farrah D, Fryer CL, Gotthelf EV, Grindlay JE, Helfand DJ, Krivonos R, Meier DL, Miller JM, Natalucci L, Ogle P, Ofek EO, Ptak A, Reynolds SP, Rigby JR, Tagliaferri G, Thorsett SE, Treister E, Urry CM (2013) The Nuclear Spectroscopic Telescope Array (NuSTAR) High-energy X-Ray Mission. ApJ770:103, DOI 10.1088/0004-637X/770/2/103, 1301.7307

Harrison RA, Sawyer EC, Carter MK, Cruise AM, Cutler RM, Fludra A, Hayes RW, Kent BJ, Lang J, Parker DJ, Payne J, Pike CD, Peskett SC, Richards AG, Gulhane JL, Norman K, Breeveld AA, Breeveld ER, Al Janabi KF, McCalden AJ, Parkinson JH, Self DG, Thomas PD, Poland 
AI, Thomas RJ, Thompson WT, Kjeldseth-Moe O, Brekke P, Karud J, Maltby P, Aschenbach B, Bräuninger H, Kühne M, Hollandt J, Siegmund OHW, Huber MCE, Gabriel AH, Mason HE, Bromage BJI (1995) The Coronal Diagnostic Spectrometer for the Solar and Heliospheric Observatory. Sol. Phys.162:233-290, DOI 10.1007/BF00733431

Harrison RA, Kent BJ, Sawyer EC, Hollandt J, Kuhne M, Paustian W, Wende B, Huber MCE (1996) The Coronal Diagnostic Spectrometer for the Solar and Heliospheric Observatory: experiment description and calibration. Metrologia 32:647-652

Hartmann J (2009) High-temperature measurement techniques for the application in photometry, radiometry and thermometry. Phys Rep 469:205-269

Hayes DS (1985) Stellar absolute fluxes and energy distributions from 0.32 to 4.0 microns. In: Hayes DS, Pasinetti LE, Philip AGD (eds) Calibration of Fundamental Stellar Quantities, IAU Symposium, vol 111, pp 225-249

Hayes DS, Latham DW (1975) A rediscussion of the atmospheric extinction and the absolute spectral-energy distribution of VEGA. ApJ197:593-601, DOI 10.1086/153548

Heise C, Kling R, Kock M, Hollandt J, Kühne M (1994) Radiometric characterization of a Penning discharge in the vacuum ultraviolet. Appl. Opt.33:5111-5117, DOI 10.1364/AO.33.005111

Henry RC, Weinstein A, Feldman PD, Fastie WG, Moos HW (1975) Low-resolution ultraviolet spectroscopy of several hot stars observed from Apollo 17. ApJ201:613-623, DOI 10.1086/153928

Hinteregger HE, Hall LA (1969) Solar extreme ultraviolet emissions in the range $2601300 \AA$ observed from OSO-III. Sol. Phys.6:175-182, DOI 10.1007/BF00150942

Hock RA, Chamberlin PC, Woods TN, Crotser D, Eparvier FG, Woodraska DL, Woods EC (2012) Extreme Ultraviolet Variability Experiment (EVE) Multiple EUV Grating Spectrographs (MEGS): Radiometric Calibrations and Results. Sol. Phys.275:145-178, DOI 10.1007/ s11207-010-9520-9

Holberg JB, Basile J, Wesemael F (1986) DA white dwarf effective temperatures determined from IUE Lyman-alpha profiles. ApJ306:629-641, DOI 10.1086/164372

Holberg JB, Ali B, Carone TE, Polidan RS (1991) Absolute far-ultraviolet spectrophotometry of hot subluminous stars from Voyager. ApJ375:716-721, DOI 10.1086/170237

Hollandt J, Kühne M, Wende B (1994) High-current hollow-cathode source as a radiant intensity standard in the 40-125-nm wavelength range. Appl. Opt.33:68-74

Hollandt J, Kuehne M, Huber MCE, Wende B (1996) Source standards for the radiometric calibration of astronomical telescopes in the VUV spectral range. A\&AS115:561-572

Hollandt J, Seidel J, Klein R, Ulm G, Migdall A, Ware M (2005) Primary sources for use in radiometry. Optical Radiometry Series: Experimental Methods in the Physical Sciences 41:213290, DOI 10.1016/S1079-4042(05)41005-X

Hovestadt D, Hilchenbach M, Bürgi A, Klecker B, Laeverenz P, Scholer M, Grünwaldt H, Axford WI, Livi S, Marsch E, Wilken B, Winterhoff HP, Ipavich FM, Bedini P, Coplan MA, Galvin AB, Gloeckler G, Bochsler P, Balsiger H, Fischer J, Geiss J, Kallenbach R, Wurz P, Reiche KU, Gliem F, Judge DL, Ogawa HS, Hsieh KC, Möbius E, Lee MA, Managadze GG, Verigin MI, Neugebauer M (1995) CELIAS - Charge, Element and Isotope Analysis System for SOHO. Sol. Phys.162:441-481, DOI 10.1007/BF00733436

Howard RA, Moses JD, Vourlidas A, Newmark JS, Socker DG, Plunkett SP, Korendyke CM, Cook JW, Hurley A, Davila JM, Thompson WT, St Cyr OC, Mentzell E, Mehalick K, Lemen JR, Wuelser JP, Duncan DW, Tarbell TD, Wolfson CJ, Moore A, Harrison RA, Waltham NR, Lang J, Davis CJ, Eyles CJ, Mapson-Menard H, Simnett GM, Halain JP, Defise JM, Mazy E, Rochus P, Mercier R, Ravet MF, Delmotte F, Auchere F, Delaboudiniere JP, Bothmer V, Deutsch W, Wang D, Rich N, Cooper S, Stephens V, Maahs G, Baugh R, McMullin D, Carter T (2008) Sun Earth Connection Coronal and Heliospheric Investigation (SECCHI). Space Sci. Rev.136:67-115, DOI 10.1007/s11214-008-9341-4 
Hoyt CC, Foukal PV (1991) Cryogenic radiometers and their application to metrology. Metrologia 28(28):163-167

Huber MCE, Dupree AK, Goldberg L, Noyes RW, Parkinson WH, Reeves EM, Withbroe GL (1973) The Harvard Experiment on OSO-6: Instrumentation, Calibration, Operation, and Description of Observations. ApJ183:291-312, DOI 10.1086/152227

Huber MCE, Reeves EM, Timothy JG (1974) Photometric Calibration of an Extreme-Ultraviolet Spectroheliometer for the Skylab Mission. In: Thompson BJ, Shannon RR (eds) Space Optics, p 33

Huber MCE, Pauluhn A, Culhane JL, Timothy JG, Wilhelm K, Zehnder A (2013) Observing Photons in Space: A Guide to Experimental Astronomy, 2nd edn. Springer, New York, Heidelberg, Dordrecht, London

Humphries CM, Jamar C, Malaise D, Wroe H (1976) Absolute calibration of the ultraviolet sky survey telescope in satellite TD 1. A\&A49:389-406

Hurwitz M, Bowyer S, Bristol R, Van Dyke Dixon W, Dupuis J, Edelstein J, Jelinsky P, Sasseen TP, Siegmund O (1998) Far-Ultraviolet Performance of the Berkeley Spectrograph during the ORFEUS-SPAS II Mission. ApJ500:L1-L7, DOI 10.1086/311388, astro-ph/9804161

Jansen F, Lumb D, Altieri B, Clavel J, Ehle M, Erd C, Gabriel C, Guainazzi M, Gondoin P, Much R, Munoz R, Santos M, Schartel N, Texier D, Vacanti G (2001) XMM-Newton observatory. I. The spacecraft and operations. A\&A365:L1-L6, DOI 10.1051/0004-6361:20000036

Kaase H, Bischoff K, Metzdorf J (1984) Quantitative Spektralradiometrie auf der Basis eines Schwarzen Strahlers hoher Temperatur und großer Strahlerfläche. Licht-Forschung 6:29-34

Kaiser ME, Kruk JW, McCandliss SR, Sahnow DJ, Rauscher BJ, Benford DJ, Bohlin RC, Deustua SE, Dixon WV, Feldman PD, Gardner JP, Kimble RA, Kurucz R, Lampton M, Moos HW, Perlmutter S, Riess AG, Woodgate BE, Wright EL (2008a) ACCESS: absolute color calibration experiment for standard stars. In: Society of Photo-Optical Instrumentation Engineers (SPIE) Conference Series, Society of Photo-Optical Instrumentation Engineers (SPIE) Conference Series, vol 7014, p 5, DOI 10.1117/12.790106

Kaiser ME, Morris MJ, Peacock GO, McCandliss SR, Rauscher BJ, Kimble RA, Kruk JW, Pelton R, Wright EL, Mott DB, Wen Y, Feldman PD, Moos HW, Riess AG, Gardner JP, Benford DJ, Woodgate BE, Bohlin R, Deustua SE, Dixon WV, Sahnow DJ, Kurucz R, Lampton M, Perlmutter S (2014) ACCESS: status and pre-flight performance. In: Society of Photo-Optical Instrumentation Engineers (SPIE) Conference Series, Society of Photo-Optical Instrumentation Engineers (SPIE) Conference Series, vol 9143, p 4, DOI 10.1117/12.2057689

Kaiser ML, Kucera TA, Davila JM, St Cyr OC, Guhathakurta M, Christian E (2008b) The STEREO Mission: An Introduction. Space Sci. Rev.136:5-16, DOI 10.1007/s11214-007-9277-0

Kanbach G, Schönfelder V, Zehnder A (2013) High-energy astrophysics - energies above $100 \mathrm{keV}$. In: Huber M, Pauluhn A, Culhane J, Timothy J, Wilhelm K, Zehnder A (eds) Observing Photons in Space, Springer, New York, Heidelberg, Dordrecht, London, pp 55-72

Kano R, Sakao T, Hara H, Tsuneta S, Matsuzaki K, Kumagai K, Shimojo M, Minesugi K, Shibasaki K, Deluca EE, Golub L, Bookbinder J, Caldwell D, Cheimets P, Cirtain J, Dennis E, Kent T, Weber M (2008) The Hinode X-Ray Telescope (XRT): Camera Design, Performance and Operations. Sol. Phys.249:263-279, DOI 10.1007/s11207-007-9058-7

Kellogg EM, Cohen LM, Edgar RJ, Evans IN, Freeman MD, Gaetz TJ, Jerius D, McDermott WC, McKinnon PJ, Murray SS, Podgorski WA, Schwartz DA, van Speybroeck LP, Wargelin BJ, Zombeck MV, Weisskopf MC, Elsner RF, O'dell SL, Tennant AF, Kolodziejczak JJ, Garmire GP, Nousek JA, Kraft S, Scholze F, Thornagel R, Ulm G, Flanagan KA, Dewey D, Bautz MW, Texter SC, Arenberg JW, Carlson R (1997) Absolute calibration of the AXAF telescope effective area. In: Hoover RB, Walker AB (eds) Grazing Incidence and Multilayer X-Ray Optical Systems, Society of Photo-Optical Instrumentation Engineers (SPIE) Conference Series, vol 3113, pp 515- 
525

Kent S, Kaiser MB, Deustua SE, Smith JA, Adelman S, Allam S, Baptista B, Bohlin RC, Clem JL, Conley A, Edelstein J, Elias J, Glass I, Henden A, Howell S, Kimble RA, Kruk JW, Lampton M, Magnier EA, McCandliss SR, Moos W, Mostek N, Mufson S, Oswalt TD, Perlmutter S, Allende Prieto C, Rauscher BJ, Riess A, Saha A, Sullivan M, Suntzeff N, Tokunaga A, Tucker D, Wing R, Woodgate B, Wright EL (2009) Photometric Calibrations for 21st Century Science. In: astro2010: The Astronomy and Astrophysics Decadal Survey, Astronomy, vol 2010, p 155, 0903.2799

Kessler MF, Steinz JA, Anderegg ME, Clavel J, Drechsel G, Estaria P, Faelker J, Riedinger JR, Robson A, Taylor BG, Ximénez de Ferrán S (1996) The Infrared Space Observatory (ISO) mission. A\&A315:L27-L31

Kim SS, Roh HS, Cho KS, Shin J (2006) Calibration of TRACE Lyman- $\alpha$ images using SOHO/SUMER observations. A\&A456:747-750, DOI 10.1051/0004-6361:20054714

Klein R, Brandt G, Fliegauf R, Hoehl A, Müller R, Thornagel R, Ulm G (2009) The Metrology Light Source operated as a primary source standard. Metrologia 46:266, DOI 10.1088/0026-1394/ $46 / 4 / \mathrm{S} 25$

Klose JZ, Bridges JM, Ott WR (1988) Radiometric calibrations of portable sources in the vacuum ultraviolet. Journal of Research 93:21-39

Kohl JL, Esser R, Gardner LD, Habbal S, Daigneau PS, Dennis EF, Nystrom GU, Panasyuk A, Raymond JC, Smith PL, Strachan L, van Ballegooijen AA, Noci G, Fineschi S, Romoli M, Ciaravella A, Modigliani A, Huber MCE, Antonucci E, Benna C, Giordano S, Tondello G, Nicolosi P, Naletto G, Pernechele C, Spadaro D, Poletto G, Livi S, von der Lühe O, Geiss J, Timothy JG, Gloeckler G, Allegra A, Basile G, Brusa R, Wood B, Siegmund OHW, Fowler W, Fisher R, Jhabvala M (1995) The Ultraviolet Coronagraph Spectrometer for the Solar and Heliospheric Observatory. Sol. Phys.162:313-356, DOI 10.1007/BF00733433

Kopp G, Heuerman K, Lawrence G (2005) The Total Irradiance Monitor (TIM): Instrument Calibration. Sol. Phys.230:111-127, DOI 10.1007/s11207-005-7447-3

Kruk JW, Kimble RA, Buss RH Jr, Davidsen AF, Durrance ST, Finley DS, Holberg JB, Kriss GA (1997) Final Astro-1 Calibration of the Hopkins Ultraviolet Telescope: A Test of White Dwarf Model Atmospheres as Far-Ultraviolet Calibration Standards. ApJ482:546-568

Kruk JW, Brown TM, Davidsen AF, Espey BR, Finley DS, Kriss GA (1999) Final Astro-2 Calibration of the Hopkins Ultraviolet Telescope. ApJS122:299-329, DOI 10.1086/313217

Kurucz RL (1979) Model atmospheres for G, F, A, B, and O stars. ApJS40:1-340, DOI 10.1086/ 190589

Kurucz RL (1993) VizieR Online Data Catalog: Model Atmospheres (Kurucz, 1979). VizieR Online Data Catalog 6039:0

Kurucz RL (2013) ATLAS12: Opacity sampling model atmosphere program. Astrophysics Source Code Library, 1303.024

Kuschnerus P, Rabus H, Richter M, Scholze F, Werner L, Ulm G (1998) Characterization of photodiodes as transfer detector standards in the $120 \mathrm{~nm}$ to $600 \mathrm{~nm}$ spectral range. Metrologia 35:355, DOI 10.1088/0026-1394/35/4/23

Lamarre JM, Dole H (2013) The Cosmic Microwave Background. In: Huber M, Pauluhn A, Culhane J, Timothy J, Wilhelm K, Zehnder A (eds) Observing Photons in Space, Springer, New York, Heidelberg, Dordrecht, London, pp 165-182

Lang J, Brown CM, Magraw JE, Payne J (1993) The Laboratory calibration of the Yohkoh Bragg crystal spectrometer. Tech. rep., Rutherford Appleton Laboratory, rAL-93-035, 59pp.

Lang J, Kent BJ, Breeveld AA, Breeveld ER, Bromage BJI, Hollandt J, Payne J, Pike CD, Thompson WT (2000) The laboratory calibration of the SOHO Coronal Diagnostic Spectrometer. Journal of Optics A: Pure and Applied Optics 2:88-106, DOI 10.1088/1464-4258/2/2/305 
Lang J, Kent BJ, Paustian W, Brown CM, Keyser C, Anderson MR, Case GCR, Chaudry RA, James AM, Korendyke CM, Pike CD, Probyn BJ, Rippington DJ, Seely JF, Tandy JA, Whillock MCR (2006) Laboratory calibration of the Extreme-Ultraviolet Imaging Spectrometer for the Solar-B satellite. Appl. Opt.45:8689-8705, DOI 10.1364/AO.45.008689

Laureijs R, Gondoin P, Duvet L, Saavedra Criado G, Hoar J, Amiaux J, Auguères JL, Cole R, Cropper M, Ealet A, Ferruit P, Escudero Sanz I, Jahnke K, Kohley R, Maciaszek T, Mellier Y, Oosterbroek T, Pasian F, Sauvage M, Scaramella R, Sirianni M, Valenziano L (2012) Euclid: ESA's mission to map the geometry of the dark universe. In: Society of Photo-Optical Instrumentation Engineers (SPIE) Conference Series, Society of Photo-Optical Instrumentation Engineers (SPIE) Conference Series, vol 8442, p 0, DOI 10.1117/12.926496

Lawrence GM, Harder JW, Rottman GJ, Woods TN, Richardson J, Mount G (1998) Stability considerations for a solar spectral intensity monitor (SIM). In: Chen PT, McClintock WE, Rottman GJ (eds) Optical Systems Contamination and Degradation, Society of Photo-Optical Instrumentation Engineers (SPIE) Conference Series, vol 3427, pp 477-485, DOI 10.1117/12.328519

Lemaire P (2002) Stellar observations. In: Pauluhn A, Huber M, von Steiger R (eds) The Radiometric Calibration of SOHO, vol 2, ESA, Noordwijk, pp 265-279, ISSI Scientific Report SR-002

Lemen JR, Title AM, Akin DJ, Boerner PF, Chou C, Drake JF, Duncan DW, Edwards CG, Friedlaender FM, Heyman GF, Hurlburt NE, Katz NL, Kushner GD, Levay M, Lindgren RW, Mathur DP, McFeaters EL, Mitchell S, Rehse RA, Schrijver CJ, Springer LA, Stern RA, Tarbell TD, Wuelser JP, Wolfson CJ, Yanari C, Bookbinder JA, Cheimets PN, Caldwell D, Deluca EE, Gates R, Golub L, Park S, Podgorski WA, Bush RI, Scherrer PH, Gummin MA, Smith P, Auker G, Jerram P, Pool P, Soufli R, Windt DL, Beardsley S, Clapp M, Lang J, Waltham N (2012) The Atmospheric Imaging Assembly (AIA) on the Solar Dynamics Observatory (SDO). Sol. Phys.275:17-40, DOI 10.1007/s11207-011-9776-8

Lin RP, Dennis BR, Hurford GJ, Smith DM, Zehnder A, Harvey PR, Curtis DW, Pankow D, Turin P, Bester M, Csillaghy A, Lewis M, Madden N, van Beek HF, Appleby M, Raudorf T, McTiernan J, Ramaty R, Schmahl E, Schwartz R, Krucker S, Abiad R, Quinn T, Berg P, Hashii M, Sterling R, Jackson R, Pratt R, Campbell RD, Malone D, Landis D, Barrington-Leigh CP, Slassi-Sennou S, Cork C, Clark D, Amato D, Orwig L, Boyle R, Banks IS, Shirey K, Tolbert AK, Zarro D, Snow F, Thomsen K, Henneck R, McHedlishvili A, Ming P, Fivian M, Jordan J, Wanner R, Crubb J, Preble J, Matranga M, Benz A, Hudson H, Canfield RC, Holman GD, Crannell C, Kosugi T, Emslie AG, Vilmer N, Brown JC, Johns-Krull C, Aschwanden M, Metcalf T, Conway A (2002) The Reuven Ramaty High-Energy Solar Spectroscopic Imager (RHESSI). Sol. Phys.210:3-32, DOI 10.1023/A:1022428818870

Lindegren L, Babusiaux C, Bailer-Jones C, Bastian U, Brown AGA, Cropper M, Høg E, Jordi C, Katz D, van Leeuwen F, Luri X, Mignard F, de Bruijne JHJ, Prusti T (2008) The Gaia mission: science, organization and present status. In: Jin WJ, Platais I, Perryman MAC (eds) IAU Symposium, IAU Symposium, vol 248, pp 217-223, DOI 10.1017/S1743921308019133

Llebaria A, Lamy PL, Bout MV (2004) Lessons learned from the SOHO/LASCO-C2 calibration. In: Fineschi S, Gummin MA (eds) Telescopes and Instrumentation for Solar Astrophysics, Society of Photo-Optical Instrumentation Engineers (SPIE) Conference Series, vol 5171, pp 26-37, DOI $10.1117 / 12.506159$

Lockwood GW, Tüg H, White NM (1992) A new solar irradiance calibration from 3295 A to 8500 A derived from absolute spectrophotometry of VEGA. ApJ390:668-678, DOI 10.1086/171318

Madden R, Canfield LR, Furst M, Hamilton A, Hughey L (1992) SURF-II: characteristics, facilities, and plans. Rev Sci Instrum 63:1594-1595

Madsen KK, Harrison FA, An H, Boggs SE, Christensen FE, Cook R, Craig WW, Forster K, Fuerst F, Grefenstette B, Hailey CJ, Kitaguchi T, Markwardt C, Mao P, Miyasaka H, Rana VR, Stern DK, Zhang WW, Zoglauer A, Walton D, Westergaard NJ (2014) The nuclear spectroscopic 
telescope array (NuSTAR) high-energy X-ray mission. In: Society of Photo-Optical Instrumentation Engineers (SPIE) Conference Series, Society of Photo-Optical Instrumentation Engineers (SPIE) Conference Series, vol 9144, p 1, DOI 10.1117/12.2056643

Mather JC, Fixsen DJ, Shafer RA, Mosier C, Wilkinson DT (1999) Calibrator Design for the COBE Far-Infrared Absolute Spectrophotometer (FIRAS). ApJ512:511-520, DOI 10.1086/306805, astro-ph/9810373

McClintock WE, Rottman GJ, Woods TN (2005a) Solar-Stellar Irradiance Comparison Experiment II (Solstice II): Instrument Concept and Design. Sol. Phys.230:225-258, DOI 10.1007/ s11207-005-7432-x

McClintock WE, Snow M, Woods TN (2005b) Solar-Stellar Irradiance Comparison Experiment II (SOLSTICE II): Pre-Launch and On-Orbit Calibrations. Sol. Phys.230:259-294, DOI 10.1007/ s11207-005-1585-5

McGraw JT, Zimmer PC, Zirzow DC, Woodward JT, Lykke KR, Cramer CE, Deustua SE, Hines DC (2012) Near-field calibration of an objective spectrophotometer to NIST radiometric standards for the creation and maintenance of standard stars for ground- and space-based applications. In: Society of Photo-Optical Instrumentation Engineers (SPIE) Conference Series, Society of Photo-Optical Instrumentation Engineers (SPIE) Conference Series, vol 8450, p 11pp, DOI $10.1117 / 12.927296$

McMullin DR, Judge DL, Hilchenbach M, Ipavich F, Bochsler P, Wurz P, Burgi A, Thompson WT, Newmark JS (2002) In-flight Comparisons of Solar EUV Irradiance Measurements Provided by the CELIAS/SEM on SOHO. ISSI Scientific Reports Series 2:135-144

Mégessier C (1995) Accuracy of the astrophysical absolute flux calibrations: visible and nearinfrared. A\&A296:771-778

Mekaoui S, Dewitte S, Conscience C, Chevalier A (2010) Total solar irradiance absolute level from DIARAD/SOVIM on the International Space Station. Advances in Space Research 45:1393-1406, DOI 10.1016/j.asr.2010.02.014

Mironov A, Zakharov A, Ambartsumyan A (2007) Improved Photometric Accuracy and the Creation of an All-sky High-Accuracy Stellar Standard System. In: Sterken C (ed) The Future of Photometric, Spectrophotometric and Polarimetric Standardization, Astronomical Society of the Pacific Conference Series, vol 364, pp 77-80

Morrill JS, Korendyke CM, Brueckner GE, Giovane F, Howard RA, Koomen M, Moses D, Plunkett SP, Vourlidas A, Esfandiari E, Rich N, Wang D, Thernisien AF, Lamy P, Llebaria A, Biesecker D, Michels D, Gong Q, Andrews M (2006) Calibration of the Soho/Lasco C3 White Light Coronagraph. Sol. Phys.233:331-372, DOI 10.1007/s11207-006-2058-1

Möstl K (1991) Radiometry. In: J Bortfeldt and B Kramer (ed) Landolt-Börnstein (New Series), Units and Fundamental Constants in Physics and Chemistry, Subvol. A, Springer, Berlin, Heidelberg, New York, pp 2-348-2-362

Mountain CM, Selby MJ, Leggett SK, Blackwell DE, Petford AD (1985) Measurement of the absolute flux from VEGA at 4.92 microns. A\&A151:399-402

Mozurkewich D, Armstrong JT, Hindsley RB, Quirrenbach A, Hummel CA, Hutter DJ, Johnston KJ, Hajian AR, Elias NM II, Buscher DF, Simon RS (2003) Angular Diameters of Stars from the Mark III Optical Interferometer. AJ126:2502-2520, DOI 10.1086/378596

Müller T, Balog Z, Nielbock M, Lim T, Teyssier D, Olberg M, Klaas U, Linz H, Altieri B, Pearson C, Bendo G, Vilenius E (2014) Herschel celestial calibration sources. Four large main-belt asteroids as prime flux calibrators for the far-IR/sub-mm range. Experimental Astronomy 37:253-330, DOI 10.1007/s10686-013-9357-y, 1311.0628

Murakami H, Baba H, Barthel P, Clements DL, Cohen M, Doi Y, Enya K, Figueredo E, Fujishiro N, Fujiwara H, Fujiwara M, Garcia-Lario P, Goto T, Hasegawa S, Hibi Y, Hirao T, Hiromoto N, Hong SS, Imai K, Ishigaki M, Ishiguro M, Ishihara D, Ita Y, Jeong WS, Jeong KS, Kaneda H, 
Kataza H, Kawada M, Kawai T, Kawamura A, Kessler MF, Kester D, Kii T, Kim DC, Kim W, Kobayashi H, Koo BC, Kwon SM, Lee HM, Lorente R, Makiuti S, Matsuhara H, Matsumoto T, Matsuo H, Matsuura S, Müller TG, Murakami N, Nagata H, Nakagawa T, Naoi T, Narita M, Noda M, Oh SH, Ohnishi A, Ohyama Y, Okada Y, Okuda H, Oliver S, Onaka T, Ootsubo T, Oyabu S, Pak S, Park YS, Pearson CP, Rowan-Robinson M, Saito T, Sakon I, Salama A, Sato S, Savage RS, Serjeant S, Shibai H, Shirahata M, Sohn J, Suzuki T, Takagi T, Takahashi H, Tanabé T, Takeuchi TT, Takita S, Thomson M, Uemizu K, Ueno M, Usui F, Verdugo E, Wada T, Wang L, Watabe T, Watarai H, White GJ, Yamamura I, Yamauchi C, Yasuda A (2007) The Infrared Astronomical Mission AKARI. PASJ59:369, DOI 10.1093/pasj/59.sp2.S369, 0708.1796

Murakami N, Kawada M, Ootsubo T, Okada Y, Takahashi H, Yasuda A, Kaneda H, Matsuo H, Baluteau JP, Davis-Imhof P, Gom BG, Naylor DA, Zavagno A, Yamamura I, Matsuura S, Shirahata M, Doi Y, Nakagawa T, Shibai H (2010) Calibration of the AKARI Far-Infrared Imaging Fourier-Transform Spectrometer. PASJ62:1155-1166, DOI 10.1093/pasj/62.5.1155, 1010.1887

Murray SS, Austin GK, Chappell JH, Gomes JJ, Kenter AT, Kraft RP, Meehan GR, Zombeck MV, Fraser GW, Serio S (2000) In-flight performance of the Chandra high-resolution camera. In: Truemper JE, Aschenbach B (eds) X-Ray Optics, Instruments, and Missions III, Society of Photo-Optical Instrumentation Engineers (SPIE) Conference Series, vol 4012, pp 68-80

Naylor DA, Baluteau JP, Bendo GJ, Benielli D, Fulton TR, Gom BG, Griffin MJ, Hopwood R, Imhof P, Lim TL, Lu N, Makiwa G, Marchili N, Orton GS, Papageorgiou A, Pearson C, Polehampton ET, Schulz B, Spencer LD, Swinyard BM, Valtchanov I, van der Wiel MHD, Veenendaal IT, Wu R (2014) In-orbit performance of the Herschel/SPIRE imaging Fourier transform spectrometer: lessons learned. In: Society of Photo-Optical Instrumentation Engineers (SPIE) Conference Series, Society of Photo-Optical Instrumentation Engineers (SPIE) Conference Series, vol 9143, p 15, DOI 10.1117/12.2054989

Neckel H, Labs D (1984) The solar radiation between 3300 and 12500 A. Sol. Phys.90:205-258, DOI 10.1007/BF00173953

Neugebauer G, Habing HJ, van Duinen R, Aumann HH, Baud B, Beichman CA, Beintema DA, Boggess N, Clegg PE, de Jong T, Emerson JP, Gautier TN, Gillett FC, Harris S, Hauser MG, Houck JR, Jennings RE, Low FJ, Marsden PL, Miley G, Olnon FM, Pottasch SR, Raimond E, Rowan-Robinson M, Soifer BT, Walker RG, Wesselius PR, Young E (1984) The Infrared Astronomical Satellite (IRAS) mission. ApJ278:L1-L6, DOI 10.1086/184209

Neupert WM, White WA, Gates WJ, Swartz M, Young RM (1969) X-ray and extreme ultraviolet (1 $400 \AA$ A) spectroscopy of the sun, from OSO-III. Sol. Phys.6:183-192, DOI 10.1007/BF00150943

Nielbock M, Müller T, Klaas U, Altieri B, Balog Z, Billot N, Linz H, Okumura K, Sánchez-Portal M, Sauvage M (2013) The Herschel PACS photometer calibration. A time dependent flux calibration for the PACS chopped point-source photometry AOT mode. Experimental Astronomy 36:631660, DOI 10.1007/s10686-013-9348-z, 1308.4068

Ogawara Y, Takano T, Kato T, Kosugi T, Tsuneta S, Watanabe T, Kondo I, Uchida Y (1991) The SOLAR-A Mission - An Overview. Sol. Phys.136:1-16, DOI 10.1007/BF00151692

Oke JB (1990) Faint spectrophotometric standard stars. AJ99:1621-1631, DOI 10.1086/115444

Oke JB, Gunn JE (1983) Secondary standard stars for absolute spectrophotometry. ApJ266:713717, DOI 10.1086/160817

Oke JB, Schild RE (1970) The Absolute Spectral Energy Distribution of Alpha Lyrae. ApJ161:1015, DOI 10.1086/150603

Pajot F, Ade PAR, Beney JL, Bréelle E, Broszkiewicz D, Camus P, Carabétian C, Catalano A, Chardin A, Charra M, Charra J, Cizeron R, Couchot F, Coulais A, Crill BP, Dassas K, Daubin J, de Bernardis P, de Marcillac P, Delouis JM, Désert FX, Duret P, Eng P, Evesque C, Fourmond JJ, François S, Giard M, Giraud-Héraud Y, Guglielmi L, Guyot G, Haissinski J, Henrot-Versillé S, Hervier V, Holmes W, Jones WC, Lamarre JM, Lami P, Lange AE, Lefebvre M, Leriche B, 
Leroy C, Macias-Perez J, Maciaszek T, Maffei B, Mahendran A, Mansoux B, Marty C, Masi S, Mercier C, Miville-Deschenes MA, Montier L, Nicolas C, Noviello F, Perdereau O, Piacentini F, Piat M, Plaszczynski S, Pointecouteau E, Pons R, Ponthieu N, Puget JL, Rambaud D, Renault C, Renault JC, Rioux C, Ristorcelli I, Rosset C, Savini G, Sudiwala R, Torre JP, Tristram M, Vallée D, Veneziani M, Yvon D (2010) Planck pre-launch status: HFI ground calibration. A\&A520:A10, DOI 10.1051/0004-6361/200913203

Pancino E, Altavilla G, Marinoni S, Cocozza G, Carrasco JM, Bellazzini M, Bragaglia A, Federici L, Rossetti E, Cacciari C, Balaguer Núñez L, Castro A, Figueras F, Fusi Pecci F, Galleti S, Gebran M, Jordi C, Lardo C, Masana E, Monguió M, Montegriffo P, Ragaini S, Schuster W, Trager S, Vilardell F, Voss H (2012) The Gaia spectrophotometric standard stars survey - I. Preliminary results. MNRAS426:1767-1781, DOI 10.1111/j.1365-2966.2012.21766.x, 1207.6042

Parmar AN, Martin DDE, Bavdaz M, Favata F, Kuulkers E, Vacanti G, Lammers U, Peacock A, Taylor BG (1997) The low-energy concentrator spectrometer on-board the BeppoSAX X-ray astronomy satellite. A\&AS122:309-326, DOI 10.1051/aas:1997137

Pauluhn A, Rüedi I, Solanki SK, Lang J, Pike CD, Schühle U, Thompson WT, Hollandt J, Huber MCE (1999) Intercalibration of SUMER and CDS on SOHO. I. SUMER detector A and CDS NIS. Appl. Opt.38:7035-7046, DOI 10.1364/AO.38.007035

Pauluhn A, Rüedi I, Solanki SK, Schühle U, Wilhelm K, Lang J, Thompson WT, Hollandt J (2001) Intercalibration of SUMER and CDS on SOHO. II. SUMER detectors A and B and CDS NIS. Appl. Opt.40:6292-6300, DOI 10.1364/AO.40.006292

Pauluhn A, Huber MCE, von Steiger R (2002) The Radiometric Calibration of SOHO. ISSI Scientific Reports Series 2

Pauluhn A, Lang J, Breeveld ER, Solanki SK, Schühle U (2003) Intercalibration of SUMER and CDS on SOHO. III. SUMER and CDS-GIS. Appl. Opt.42:657-666, DOI 10.1364/AO.42.000657

Penzias AA, Wilson RW (1966) Determination of the Microwave Spectrum of Galactic Radiation. ApJ146:666, DOI 10.1086/148944

Pesnell WD, Thompson BJ, Chamberlin PC (2012) The Solar Dynamics Observatory (SDO). Sol. Phys.275:3-15, DOI 10.1007/s11207-011-9841-3

Peterson DM, Hummel CA, Pauls TA, Armstrong JT, Benson JA, Gilbreath GC, Hindsley RB, Hutter DJ, Johnston KJ, Mozurkewich D, Schmitt HR (2006) Vega is a rapidly rotating star. Nature440:896-899, DOI 10.1038/nature04661, astro-ph/0603520

Pilbratt GL (2008) Herschel mission overview and key programmes. In: Society of Photo-Optical Instrumentation Engineers (SPIE) Conference Series, Society of Photo-Optical Instrumentation Engineers (SPIE) Conference Series, vol 7010, p 12pp, DOI 10.1117/12.789431

Planck Collaboration, Adam R, Ade PAR, Aghanim N, Arnaud M, Ashdown M, Aumont J, Baccigalupi C, Banday AJ, Barreiro RB, et al (2015a) Planck 2015 results. VIII. High Frequency Instrument data processing: Calibration and maps. ArXiv e-prints p 26, 1502.01587

Planck Collaboration, Ade PAR, Aghanim N, Ashdown M, Aumont J, Baccigalupi C, Banday AJ, Barreiro RB, Bartolo N, Battaglia P, et al (2015b) Planck 2015 results. V. LFI calibration. ArXiv e-prints p 22, 1505.08022

Poglitsch A, Waelkens C, Geis N, Feuchtgruber H, Vandenbussche B, Rodriguez L, Krause O, Renotte E, van Hoof C, Saraceno P, Cepa J, Kerschbaum F, Agnèse P, Ali B, Altieri B, Andreani P, Augueres JL, Balog Z, Barl L, Bauer OH, Belbachir N, Benedettini M, Billot N, Boulade O, Bischof H, Blommaert J, Callut E, Cara C, Cerulli R, Cesarsky D, Contursi A, Creten Y, De Meester W, Doublier V, Doumayrou E, Duband L, Exter K, Genzel R, Gillis JM, Grözinger U, Henning T, Herreros J, Huygen R, Inguscio M, Jakob G, Jamar C, Jean C, de Jong J, Katterloher R, Kiss C, Klaas U, Lemke D, Lutz D, Madden S, Marquet B, Martignac J, Mazy A, Merken P, Montfort F, Morbidelli L, Müller T, Nielbock M, Okumura K, Orfei R, Ottensamer R, Pezzuto S, Popesso P, Putzeys J, Regibo S, Reveret V, Royer P, Sauvage M, Schreiber J, Stegmaier 
J, Schmitt D, Schubert J, Sturm E, Thiel M, Tofani G, Vavrek R, Wetzstein M, Wieprecht E, Wiezorrek E (2010) The Photodetector Array Camera and Spectrometer (PACS) on the Herschel Space Observatory. A\&A518:L2, DOI 10.1051/0004-6361/201014535, 1005.1487

Price SD, Paxson C, Engelke C, Murdock TL (2004) Spectral Irradiance Calibration in the Infrared. XV. Absolute Calibration of Standard Stars by Experiments on the Midcourse Space Experiment. AJ128:889-910, DOI 10.1086/422024

Quinn TJ (1997) Primary methods of measurement and primary standards. Metrologia 34:61-65, DOI 10.1088/0026-1394/34/1/9

Quinn TJ, Martin JE (1991a) Cryogenic radiometry, prospects for further improvements in accuracy. Metrologia 28(28):155-161

Quinn TJ, Martin JE (1991b) Cryogenic radiometry: the problem of hydrogen condensation in detectors operated at temperatures below 4 K. Appl Opt 30(16):2065-2067, DOI 10.1364/AO. 30.002065, URL http://ao.osa.org/abstract.cfm?URI=ao-30-16-2065

Reber CA, Trevathan CE, McNeal RJ, Luther MR (1993) The Upper Atmosphere Research Satellite (UARS) mission. J. Geophys. Res.98:10,643-10,647, DOI 10.1029/92JD02828

Reeves EM, Parkinson WH (1970) Calibration changes in EUV solar satellite instruments. Appl. Opt.9:1201-1208, DOI 10.1364/AO.9.001201

Reeves EM, Timothy JG, Huber MCE (1977a) Extreme UV spectroheliometer on the Apollo Telescope Mount. Appl. Opt.16:837-848

Reeves EM, Timothy JG, Withbroe GL, Huber MCE (1977b) Photometric calibration of the EUV spectroheliometer on ATM. Appl. Opt.16:849-857

Rieke GH, Blaylock M, Decin L, Engelbracht C, Ogle P, Avrett E, Carpenter J, Cutri RM, Armus L, Gordon K, Gray RO, Hinz J, Su K, Willmer CNA (2008) Absolute Physical Calibration in the Infrared. AJ135:2245-2263, DOI 10.1088/0004-6256/135/6/2245, 0806.1910

Rosa MR (1997) Physical Modeling of Scientific Instruments. In: Hunt G, Payne H (eds) Astronomical Data Analysis Software and Systems VI, Astronomical Society of the Pacific Conference Series, vol 125, p 411

Rothschild RE, Blanco PR, Gruber DE, Heindl WA, MacDonald DR, Marsden DC, Pelling MR, Wayne LR, Hink PL (1998) In-Flight Performance of the High-Energy X-Ray Timing Experiment on the Rossi X-Ray Timing Explorer. ApJ496:538-549, DOI 10.1086/305377, astro-ph/9710328

Rottman G, Mount G, Lawrence G, Woods T, Harder J, Tournois S (1998) Solar Spectral Irradiance Measuremetns: Visible to Near-Infrared Regions. Metrologia 35:707-712, DOI $10.1088 / 0026-1394 / 35 / 4 / 82$

Rottman GJ, Woods TN (1994) Upper Atmosphere Research Satellite (UARS) Solar Stellar Irradiance Comparison Experiment (SOLSTICE). In: Wang J, Hays PB (eds) Optical Spectroscopic Techniques and Instrumentation for Atmospheric and Space Research, Society of Photo-Optical Instrumentation Engineers (SPIE) Conference Series, vol 2266, pp 317-327

Sahnow DJ, Moos HW, Ake TB, Andersson BG, Andre M, Artis D, Berman AF, Blair WP, Brownsberger KR, Calvani HM, Chayer P, Conard SJ, Feldman PD, Friedman SD, Fullerton AW, Gaines GA, Green JC, Gummin MA, Joyce JB, Kaiser ME, Kruk JW, Lindler DJ, Massa D, Murphy EM, Oegerle WR, Ohl RG, Osterman SN, Roberts BA, Roth KC, Sankrit R, Sembach KR, Shelton RL, Siegmund OH, Weaver HA, Wilkinson E (2000a) On-orbit performance of the Far Ultraviolet Spectroscopic Explorer (FUSE). In: Breckinridge JB, Jakobsen P (eds) UV, Optical, and IR Space Telescopes and Instruments, Society of Photo-Optical Instrumentation Engineers (SPIE) Conference Series, vol 4013, pp 334-343

Sahnow DJ, Moos HW, Friedman SD, Blair WP, Conard SJ, Kruk JW, Murphy EM, Oegerle WR, Ake TB (2000b) Far Ultraviolet Spectroscopic Explorer: one year in orbit. In: Fineschi S, Korendyke CM, Siegmund $\mathrm{OH}$, Woodgate BE (eds) Instrumentation for UV/EUV Astronomy and Solar Missions, Society of Photo-Optical Instrumentation Engineers (SPIE) Conference Series, 
vol 4139, pp 131-136

Saloman EB (1978) Typical photoefficiency between 20-250 eV of windowless XUV photodiodes with tungsten and anodized aluminum oxide photocathodes. Appl. Opt.17:1489-1490, DOI 10.1364/AO.17.001489

Samson JA, Ederer DL (2000) Vacuum Ultraviolet Spectroscopy. Academic Press, London, Amsterdam, Oxford, San Diego

Samson JAR (1964) Photoionization cross sections of xenon from the $2 \mathrm{p} \frac{1}{2}$ edge to 280 å. J Opt Soc Am 54(6):842-842, DOI 10.1364/JOSA.54.000842, URL http://www.osapublishing.org/abstract. cfm?URI=josa-54-6-842

Samson JAR, Haddad GN (1974) Absolute photon-flux measurements in the vacuum ultraviolet. J Opt Soc Am 64(1):47-54, DOI 10.1364/JOSA.64.000047, URL http://www.osapublishing.org/ abstract.cfm?URI=josa-64-1-47

Santangelo AE, Piraino S, Segreto A, Fazio G, Giarrusso S (1997) In-orbit performance and calibration of the High-Pressure Gas Scintillation Proportional Counter on board the BeppoSAX satellite. In: Siegmund OH, Gummin MA (eds) EUV, X-Ray, and Gamma-Ray Instrumentation for Astronomy VIII, Society of Photo-Optical Instrumentation Engineers (SPIE) Conference Series, vol 3114, pp 216-227

Schläppi B, Altwegg K, Balsiger H, Hässig M, Jäckel A, Wurz P, Fiethe B, Rubin M, Fuselier SA, Berthelier JJ, de Keyser J, RèMe H, Mall U (2010) Influence of spacecraft outgassing on the exploration of tenuous atmospheres with in situ mass spectrometry. Journal of Geophysical Research (Space Physics) 115(A14):A12313, DOI 10.1029/2010JA015734

Schmidtke G (2015) Extreme ultraviolet spectral irradiance measurements since 1946. Hist Geo Space Sci 6:3-22, DOI 10.5194/hgss-6-3-2015

Schmidtke G, Brunner R, Eberhard D, Halford B, Klocke U, Knothe M, Konz W, Riedel WJ, Wolf H (2006) SOL ACES: Auto-calibrating EUV/UV spectrometers for measurements onboard the International Space Station. Advances in Space Research 37:273-282, DOI 10.1016/j.asr.2005. 01.112

Schmidtke G, Nikutowski B, Jacobi C, Brunner R, Erhardt C, Knecht S, Scherle J, Schlagenhauf J (2014) Solar EUV Irradiance Measurements by the Auto-Calibrating EUV Spectrometers (SolACES) Aboard the International Space Station (ISS). Sol. Phys.289:1863-1883, DOI 10.1007/s11207-013-0430-5

Schönfelder V, Kanbach G (2013) Imaging through Compton scattering and pair creation. In: Huber M, Pauluhn A, Culhane J, Timothy J, Wilhelm K, Zehnder A (eds) Observing Photons in Space, Springer, New York, Heidelberg, Dordrecht, London, pp 225-242

Schou J, Scherrer PH, Bush RI, Wachter R, Couvidat S, Rabello-Soares MC, Bogart RS, Hoeksema JT, Liu Y, Duvall TL, Akin DJ, Allard BA, Miles JW, Rairden R, Shine RA, Tarbell TD, Title AM, Wolfson CJ, Elmore DF, Norton AA, Tomczyk S (2012) Design and Ground Calibration of the Helioseismic and Magnetic Imager (HMI) Instrument on the Solar Dynamics Observatory (SDO). Sol. Phys.275:229-259, DOI 10.1007/s11207-011-9842-2

Schühle U, Curdt W, Hollandt J, Feldman U, Lemaire P, Wilhelm K (2000) Radiometric Calibration of the Vacuum-Ultraviolet Spectrograph SUMER on the SOHO Spacecraft with the B Detector. Appl. Opt.39:418-425, DOI 10.1364/AO.39.000418

Schwartz DA, David LP, Donnelly RH, Edgar RJ, Gaetz TJ, Graessle DE, Jerius D, Juda M, Kellogg EM, McNamara BR, Plucinsky PP, Van Speybroeck LP, Wargelin BJ, Wolk S, Zhao P, Dewey D, Marshall HL, Schulz NS, Elsner RF, Kolodziejczak JJ, O’Dell SL, Swartz DA, Tennant AF, Weisskopf MC (2000) Absolute effective area of the Chandra high-resolution mirror assembly (HRMA). In: Truemper JE, Aschenbach B (eds) X-Ray Optics, Instruments, and Missions III, Society of Photo-Optical Instrumentation Engineers (SPIE) Conference Series, vol 4012, pp 2840 
Schwinger J (1949) On the classical radiation of accelerated electrons. Phys Rev 75:1912-1925, DOI 10.1103/PhysRev.75.1912, URL http://link.aps.org/doi/10.1103/PhysRev.75.1912

Seely JF, Brown CM, Windt DL, Donguy S, Kjornrattanawanich B (2004) Normal-Incidence Efficiencies of Multilayer-Coated Laminar Gratings for the Extreme-Ultraviolet Imaging Spectrometer on the Solar-B Mission. Appl. Opt.43:1463-1471, DOI 10.1364/AO.43.001463

Selby MJ, Mountain CM, Blackwell DE, Petford AD, Leggett SK (1983) Measurement of the absolute monochromatic flux from VEGA at 2.20 and 3.80 microns by comparison with a furnace. MNRAS203:795-800

Sembach K (2015) Towards a 2020 Vision for the Hubble Space Telescope. Tech. rep., STScI, Newsletter 32/01:1-3

Sembay S, Guainazzi M, Plucinsky P, Nevalainen J (2010) Defining High-Energy Calibration Standards: IACHEC (International Astronomical Consortium for High-Energy Calibration). X-ray Astronomy 2009; Present Status, Multi-Wavelength Approach and Future Perspectives 1248:593594, DOI 10.1063/1.3475350

Sing D, Holberg JB, Dupuis J (2002) A Comparison of EUVE Fluxes with Absolute Stellar Calibration at Longer Wavelengths. In: Howell SB, Dupuis J, Golombek D, Walter FM, Cullison $\mathrm{J}$ (eds) Continuing the Challenge of EUV Astronomy: Current Analysis and Prospects for the Future, Astronomical Society of the Pacific Conference Series, vol 264, p 57

Sirk MM, Vallerga JV, Finley DS, Jelinsky P, Malina RF (1997) Performance of the Extreme Ultraviolet Explorer Imaging Telescopes. ApJS110:347-356, DOI 10.1086/313002

Smith AW, Woodward JT, Jenkins CA, Brown SW, Lykke KR (2009) Absolute flux calibration of stars: calibration of the reference telescope. Metrologia 46:219-223, DOI 10.1088/0026-1394/ $46 / 4 / \mathrm{S} 16$

Smith DM, Lin RP, Turin P, Curtis DW, Primbsch JH, Campbell RD, Abiad R, Schroeder P, Cork CP, Hull EL, Landis DA, Madden NW, Malone D, Pehl RH, Raudorf T, Sangsingkeow P, Boyle R, Banks IS, Shirey K, Schwartz R (2002) The RHESSI Spectrometer. Sol. Phys.210:33-60, DOI 10.1023/A:1022400716414

Smith PL, Huber MCE, Parkinson WH, Kuhne M, Kock M (1991) In-Orbit Extreme Ultraviolet Radiometric Calibration of Satellite Instrumentation. In: Malina RF, Bowyer S (eds) Extreme Ultraviolet Astronomy, p 390

Snow M, Reberac A, Quémerais E, Clarke J, McClintock WE, Woods TN (2013) A New Catalog of Ultraviolet Stellar Spectra for Calibration, Springer, New York, pp 191-226. DOI 10.1007/ 978-1-4614-6384-9_7, edited by E Quémerais, M. Snow and R.-M. Bonnet

Stock M, Fischer J, Friedrich R, Jung HJ, Thornagel R, Ulm G, Wende B (1993) Present State of the Comparison between Radiometric Scales Based on Three Primary Standards. Metrologia 30:439-449, DOI 10.1088/0026-1394/30/4/041

Strongylis GJ, Bohlin RC (1979) Absolute calibration in the 1750 A-3350 A region. PASP91:205213, DOI 10.1086/130472

Strüder L, Briel U, Dennerl K, Hartmann R, Kendziorra E, Meidinger N, Pfeffermann E, Reppin C, Aschenbach B, Bornemann W, Bräuninger H, Burkert W, Elender M, Freyberg M, Haberl F, Hartner G, Heuschmann F, Hippmann H, Kastelic E, Kemmer S, Kettenring G, Kink W, Krause N, Müller S, Oppitz A, Pietsch W, Popp M, Predehl P, Read A, Stephan KH, Stötter D, Trümper J, Holl P, Kemmer J, Soltau H, Stötter R, Weber U, Weichert U, von Zanthier C, Carathanassis D, Lutz G, Richter RH, Solc P, Böttcher H, Kuster M, Staubert R, Abbey A, Holland A, Turner M, Balasini M, Bignami GF, La Palombara N, Villa G, Buttler W, Gianini F, Lainé R, Lumb D, Dhez P (2001) The European Photon Imaging Camera on XMM-Newton: The pn-CCD camera. A\&A365:L18-L26, DOI 10.1051/0004-6361:20000066

Sullivan M, Guy J, Conley A, Regnault N, Astier P, Balland C, Basa S, Carlberg RG, Fouchez D, Hardin D, Hook IM, Howell DA, Pain R, Palanque-Delabrouille N, Perrett KM, Pritchet 
CJ, Rich J, Ruhlmann-Kleider V, Balam D, Baumont S, Ellis RS, Fabbro S, Fakhouri HK, Fourmanoit N, González-Gaitán S, Graham ML, Hudson MJ, Hsiao E, Kronborg T, Lidman C, Mourao AM, Neill JD, Perlmutter S, Ripoche P, Suzuki N, Walker ES (2011) SNLS3: Constraints on Dark Energy Combining the Supernova Legacy Survey Three-year Data with Other Probes. ApJ737:102, DOI 10.1088/0004-637X/737/2/102, 1104.1444

Swinyard B, Wild W (2013) Far-infrared imaging and spectroscopic instrumentation. In: Huber M, Pauluhn A, Culhane J, Timothy J, Wilhelm K, Zehnder A (eds) Observing Photons in Space, Springer, New York, Heidelberg, Dordrecht, London, pp 261-282

Swinyard BM, Polehampton ET, Hopwood R, Valtchanov I, Lu N, Fulton T, Benielli D, Imhof P, Marchili N, Baluteau JP, Bendo GJ, Ferlet M, Griffin MJ, Lim TL, Makiwa G, Naylor DA, Orton GS, Papageorgiou A, Pearson CP, Schulz B, Sidher SD, Spencer LD, van der Wiel MHD, Wu R (2014) Calibration of the Herschel SPIRE Fourier Transform Spectrometer. MNRAS440:36583674, DOI 10.1093/mnras/stu409, 1403.1107

Takita S, Ikeda N, Kitamura Y, Ishihara D, Kataza H, Kawamura A, Oyabu S, Ueno M, Yamamura I (2012) Slow-Scan Observations with the Infrared Camera (IRC) aboard AKARI. PASJ64:126, DOI 10.1093/pasj/64.6.126, 1209.0931

Taubert RD, Monte C, Baltruschat C, Schirmacher A, Gutschwager B, Hartmann J, Hollandt J, Kochems D, Küchel C, te Plate M (2009) The spectral photon flux of the radiometric calibration spectral source for the NIRSpec instrument of the James Webb Space Telescope. Metrologia 46:207, DOI 10.1088/0026-1394/46/4/S14

Taylor BG, Andresen RD, Peacock A, Zobl R (1981) The EXOSAT mission. Space Sci. Rev.30:479494, DOI 10.1007/BF01246069

Thekaekara MP (1974) Extraterrestrial solar spectrum, 3000 - $6100 \AA$ at $1-\AA$ intervals. Appl. Opt.13:518-522, DOI 10.1364/AO.13.000518

Thernisien AF, Morrill JS, Howard RA, Wang D (2006) Photometric Calibration of the Lasco-C3 Coronagraph Using Stars. Sol. Phys.233:155-169, DOI 10.1007/s11207-006-2047-4

Thomas R (2002) 20:20 Vision and SOHO Cleanliness. ISSI Scientific Reports Series 2:91-104

Thompson WT, Reginald NL (2008) The Radiometric and Pointing Calibration of SECCHI COR1 on STEREO. Sol. Phys.250:443-454, DOI 10.1007/s11207-008-9228-2

Thompson WT, McMullin DR, Newmark JS (2002) Comparison of CDS Irradiance Measurements with SEM and EIT. ISSI Scientific Reports Series 2:211-223

Thornagel R, Fischer J, Friedrich R, Stock M, Ulm G, Wende B (1996) The electron storage ring BESSY as a primary standard source - a radiometric comparison with a cryogenic electrical substitution radiometer in the visible. Metrologia 32:459

Thuillier G, Floyd L, Woods TN, Cebula R, Hilsenrath E, Hersé M, Labs D (2004a) Solar Irradiance Reference Spectra. In: Pap JM, Fox P, Frohlich C, Hudson HS, Kuhn J, McCormack J, North G, Sprigg W, Wu ST (eds) Solar Variability and its Effects on Climate. Geophysical Monograph 141, Washington DC American Geophysical Union Geophysical Monograph Series, vol 141, pp 171-194

Thuillier G, Floyd L, Woods TN, Cebula R, Hilsenrath E, Hersé M, Labs D (2004b) Solar irradiance reference spectra for two solar active levels. Advances in Space Research 34:256-261, DOI 10.1016/j.asr.2002.12.004

Thuillier G, Foujols T, Bolsée D, Gillotay D, Hersé M, Peetermans W, Decuyper W, Mandel H, Sperfeld P, Pape S, Taubert DR, Hartmann J (2009) SOLAR/SOLSPEC: Scientific Objectives, Instrument Performance and Its Absolute Calibration Using a Blackbody as Primary Standard Source. Sol. Phys.257:185-213, DOI 10.1007/s11207-009-9361-6

Timothy JG, Chambers RM, d'Entremont AM, Lanham NW, Reeves EM (1975) A sounding rocket spectroheliometer for photometric studies at extreme ultraviolet wavelengths. Space Science Instrumentation 1:23-49 
Tüg H, White NM, Lockwood GW (1977) Absolute energy distributions of Alpha Lyrae and 109 Virginis from 3295 A to 9040 A. A\&A61:679-684

Turner MJL, Abbey A, Arnaud M, Balasini M, Barbera M, Belsole E, Bennie PJ, Bernard JP, Bignami GF, Boer M, Briel U, Butler I, Cara C, Chabaud C, Cole R, Collura A, Conte M, Cros A, Denby M, Dhez P, Di Coco G, Dowson J, Ferrando P, Ghizzardi S, Gianotti F, Goodall CV, Gretton L, Griffiths RG, Hainaut O, Hochedez JF, Holland AD, Jourdain E, Kendziorra E, Lagostina A, Laine R, La Palombara N, Lortholary M, Lumb D, Marty P, Molendi S, Pigot C, Poindron E, Pounds KA, Reeves JN, Reppin C, Rothenflug R, Salvetat P, Sauvageot JL, Schmitt D, Sembay S, Short ADT, Spragg J, Stephen J, Strüder L, Tiengo A, Trifoglio M, Trümper J, Vercellone S, Vigroux L, Villa G, Ward MJ, Whitehead S, Zonca E (2001) The European Photon Imaging Camera on XMM-Newton: The MOS cameras. A\&A365:L27-L35, DOI 10.1051/0004-6361:20000087, astro-ph/0011498

VanHoosier ME, Bartoe JDF, Brueckner GE, Prinz DK (1988) Absolute solar spectral irradiance $120 \mathrm{~nm}$ - $400 \mathrm{~nm}$ (Results from the Solar Ultraviolet Spectral Irradiance Monitor-SUSIMExperiment on board Spacelab 2). Astrophysical Letters and Communications 27:163-168

von der Linden W, Dose V, von Toussaint U (2014) Bayesian Probability Theory. Cambridge University Press, Cambridge, UK

Wang T, Thomas RJ, Brosius JW, Young PR, Rabin DM, Davila JM, Del Zanna G (2011) Underflight Calibration of SOHO/CDS and Hinode/EIS with EUNIS-07. ApJS197:32, DOI 10.1088/0067-0049/197/2/32, 1109.6598

Waters WR, Walker JH, Hattenburg AT (1988) The NBS scale of radiance temperature. Tech. rep., NBS/NIST

Weisskopf MC, Tananbaum HD, Van Speybroeck LP, O’Dell SL (2000) Chandra X-ray Observatory (CXO): overview. In: Truemper JE, Aschenbach B (eds) X-Ray Optics, Instruments, and Missions III, Society of Photo-Optical Instrumentation Engineers (SPIE) Conference Series, vol 4012, pp 2-16, astro-ph/0004127

Weisskopf MC, Brinkman B, Canizares C, Garmire G, Murray S, Van Speybroeck LP (2002) An Overview of the Performance and Scientific Results from the Chandra X-Ray Observatory. PASP114:1-24, DOI 10.1086/338108, astro-ph/0110308

Wild W (2013) Coherent far-infrared / submillimetre detectors. In: Huber M, Pauluhn A, Culhane J, Timothy J, Wilhelm K, Zehnder A (eds) Observing Photons in Space, Springer, New York, Heidelberg, Dordrecht, London, pp 543-564

Wilhelm K (2006) Solar VUV measurements obtained by SOHO instruments and their radiometric calibration. Advances in Space Research 37:225-233, DOI 10.1016/j.asr.2004.12.057

Wilhelm K (2010) Quantitative solar spectroscopy. Astronomische Nachrichten 331:502-511, DOI 10.1002 /asna.200911360

Wilhelm K, Fröhlich C (2013) Photons - from source to detector. In: Huber M, Pauluhn A, Culhane J, Timothy J, Wilhelm K, Zehnder A (eds) Observing Photons in Space, Springer, New York, Heidelberg, Dordrecht, London, pp 21-53

Wilhelm K, Curdt W, Marsch E, Schühle U, Lemaire P, Gabriel A, Vial JC, Grewing M, Huber MCE, Jordan SD, Poland AI, Thomas RJ, Kühne M, Timothy JG, Hassler DM, Siegmund OHW (1995) SUMER - Solar Ultraviolet Measurements of Emitted Radiation. Sol. Phys.162:189-231, DOI 10.1007/BF00733430

Wilhelm K, Lemaire P, Feldman U, Hollandt J, Schühle U, Curdt W (1997) Radiometric calibration of SUMER: refinement of the laboratory results under operational conditions on SOHO. Appl. Opt.36:6416-6422, DOI 10.1364/AO.36.006416

Wilhelm K, Schühle U, Curdt W, Dammasch IE, Hollandt J, Lemaire P, Huber MCE (2002) Solar Vacuum-ultraviolet Radiometry with SUMER. In: Pauluhn A, Huber M, von Steiger R (eds) The Radiometric Calibration of $S O H O$, vol 2, ESA, Noordwijk, pp 145-160, ISSI Scientific Report 
SR-002

Willson RC (1999) Solar Irradiance Variations. In: Strong KT, Saba JLR, Haisch BM, Schmelz JT (eds) The many faces of the sun: a summary of the results from NASA's Solar Maximum Mission., p 19

Willson RC (2014) ACRIM3 and the Total Solar Irradiance database. Ap\&SS352:341-352, DOI $10.1007 /$ s10509-014-1961-4

Woods TN, Rottman G (2005) XUV Photometer System (XPS): Solar Variations during the SORCE Mission. Sol. Phys.230:375-387, DOI 10.1007/s11207-005-2555-7

Woods TN, Rottman GJ, Ucker GJ (1993) Solar-Stellar Irradiance Comparison Experiment 1. II - Instrument calibrations. J. Geophys. Res.98:10,679-10,694, DOI 10.1029/93JD00463

Woods TN, Prinz DK, Rottman GJ, London J, Crane PC, Cebula RP, Hilsenrath E, Brueckner GE, Andrews MD, White OR, VanHoosier ME, Floyd LE, Herring LC, Knapp BG, Pankratz CK, Reiser PA (1996) Validation of the UARS solar ultraviolet irradiances: Comparison with the ATLAS 1 and 2 measurements. J. Geophys. Res.101:9541-9570, DOI 10.1029/96JD00225

Woods TN, Bailey SM, Eparvier FG, Lawrence GM, Lean J, McClintock WE, Roble RG, Rottman GJ, Solomon SC, Tobiska WK, Ucker GJ, White OR (1998) TIMED solar EUV experiment. In: Korendyke CM (ed) Missions to the Sun II, Society of Photo-Optical Instrumentation Engineers (SPIE) Conference Series, vol 3442, pp 180-191

Woods TN, Rottman G, Vest R (2005) XUV Photometer System (XPS): Overview and Calibrations. Sol. Phys.230:345-374, DOI 10.1007/s11207-005-4119-2

Woods TN, Chamberlin PC, Harder JW, Hock RA, Snow M, Eparvier FG, Fontenla J, McClintock WE, Richard EC (2009) Solar Irradiance Reference Spectra (SIRS) for the 2008 Whole Heliosphere Interval (WHI). Geophys. Res. Lett.36:L01101, DOI 10.1029/2008GL036373

Woods TN, Eparvier FG, Hock R, Jones AR, Woodraska D, Judge D, Didkovsky L, Lean J, Mariska J, Warren H, McMullin D, Chamberlin P, Berthiaume G, Bailey S, Fuller-Rowell T, Sojka J, Tobiska WK, Viereck R (2012) Extreme Ultraviolet Variability Experiment (EVE) on the Solar Dynamics Observatory (SDO): Overview of Science Objectives, Instrument Design, Data Products, and Model Developments. Sol. Phys.275:115-143, DOI 10.1007/s11207-009-9487-6

Yamamura I, Makiuti S, Ikeda N, Fukuda Y, Oyabu S, Koga T, White GJ (2010) VizieR Online Data Catalog: AKARI/FIS All-Sky Survey Point Source Catalogues (ISAS/JAXA, 2010). VizieR Online Data Catalog 2298:37pp

Yeo K, Krivova N, Solanki S (2015) Solar cycle variation in solar irradiance. In: Balogh A, Hudson H, Petrovay K, von Steiger R (eds) The Solar Activity Cycle, Space Sciences Series of ISSI, vol 53, Springer New York, pp 137-167, DOI 10.1007/978-1-4939-2584-1_5, URL http://dx.doi. org/10.1007/978-1-4939-2584-1_5 